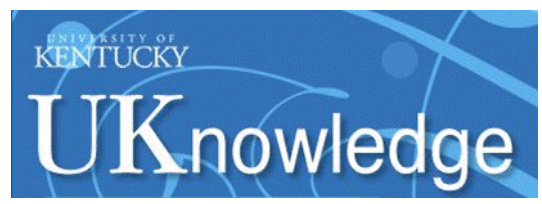

University of Kentucky

UKnowledge

6-11-2014

\title{
Optical-Faint, Far-Infrared-Bright Herschel Sources in the CANDELS Fields: Ultra-Luminous Infrared Galaxies at $z>1$ and the Effect of Source Blending
}

\author{
Haojing Yan \\ University of Missouri \\ Mauro Stefanon \\ University of Missouri \\ Zhiyuan Ma \\ University of Missouri \\ S. P. Willner \\ Harvard-Smithsonian Center for Astrophysics \\ Rachel Somerville \\ Rutgers University \\ Follow this and additional works at: https://uknowledge.uky.edu/physastron_facpub \\ See rart of the Astrophysics for additional authortsonomy Commons, and the Physics Commons \\ Right click to open a feedback form in a new tab to let us know how this document benefits you.
}

\section{Repository Citation}

Yan, Haojing; Stefanon, Mauro; Ma, Zhiyuan; Willner, S. P.; Somerville, Rachel; Ashby, Matthew L.N.; Davé, Romeel; Pérez-González, Pablo G.; Cava, Antonio; Wiklind, Tommy; Kocevski, Dale D.; Rafelski, Marc; Kartaltepe, Jeyhan; Cooray, Asantha; Koekemoer, Anton M.; and Grogin, Norman A., "Optical-Faint, FarInfrared-Bright Herschel Sources in the CANDELS Fields: Ultra-Luminous Infrared Galaxies at $z>1$ and the Effect of Source Blending" (2014). Physics and Astronomy Faculty Publications. 266.

https://uknowledge.uky.edu/physastron_facpub/266

This Article is brought to you for free and open access by the Physics and Astronomy at UKnowledge. It has been accepted for inclusion in Physics and Astronomy Faculty Publications by an authorized administrator of UKnowledge. For more information, please contact UKnowledge@lsv.uky.edu. 
Optical-Faint, Far-Infrared-Bright Herschel Sources in the CANDELS Fields: UltraLuminous Infrared Galaxies at $z>1$ and the Effect of Source Blending

\author{
Digital Object Identifier (DOI) \\ http://dx.doi.org/10.1088/0067-0049/213/1/2 \\ Notes/Citation Information \\ Published in The Astrophysical Journal Supplement Series, v. 213, no. 1, article 2, p. 1-40. \\ (C) 2014. The American Astronomical Society. All rights reserved.
}

Reproduced by permission of the AAS.

Authors

Haojing Yan, Mauro Stefanon, Zhiyuan Ma, S. P. Willner, Rachel Somerville, Matthew L.N. Ashby, Romeel Davé, Pablo G. Pérez-González, Antonio Cava, Tommy Wiklind, Dale D. Kocevski, Marc Rafelski, Jeyhan Kartaltepe, Asantha Cooray, Anton M. Koekemoer, and Norman A. Grogin 


\title{
OPTICAL-FAINT, FAR-INFRARED-BRIGHT HERSCHEL SOURCES IN THE CANDELS FIELDS: ULTRA-LUMINOUS INFRARED GALAXIES AT $z>1$ AND THE EFFECT OF SOURCE BLENDING
}

\author{
Haojing Yan $^{1}$, Mauro Stefanon ${ }^{1}$, Zhiyuan Ma ${ }^{1}$, S. P. Willner ${ }^{2}$, Rachel Somerville ${ }^{3}$, Matthew L. N. Ashby ${ }^{2}$, \\ Romeel Davé ${ }^{4}$, Pablo G. Pérez-González ${ }^{5}$, Antonio Cava ${ }^{6}$, Tommy Wiklind ${ }^{7}$, Dale Kocevski ${ }^{8}$, Marc Rafelski ${ }^{9}$, \\ Jeyhan Kartaltepe $^{10,13}$, Asantha Cooray ${ }^{11}$, Anton M. Koekemoer ${ }^{12}$, and Norman A. Grogin ${ }^{12}$ \\ ${ }^{1}$ Department of Physics \& Astronomy, University of Missouri, Columbia, MO 65211, USA \\ ${ }^{2}$ Harvard-Smithsonian Center for Astrophysics, 60 Garden Street, Cambridge, MA 02138, USA \\ ${ }^{3}$ Department of Physics and Astronomy, Rutgers University, 136 Frelinghuysen Road, Piscataway, NJ 08854, USA \\ ${ }^{4}$ University of the Western Cape, 7535 Bellville, Cape Town, South Africa \\ ${ }_{5}^{5}$ Departamento de Astrofísica, Facultad de CC. Físicas, Universidad Complutense de Madrid, E-28040 Madrid, Spain \\ ${ }^{6}$ Observatoire de Genève, Université de Genève, 51 Ch. des Maillettes, 1290 Versoix, Switzerland \\ 7 Joint ALMA Observatory, Alonso de Cordova 3107, Vitacura, Santiago, Chile \\ ${ }^{8}$ Department of Physics and Astronomy, University of Kentucky, Lexington, KY 40506, USA \\ ${ }^{9}$ Infrared Processing and Analysis Center, California Institute of Technology, Pasadena, CA 91125, USA \\ ${ }^{10}$ National Optical Astronomy Observatory, 950 North Cherry Avenue, Tucson, AZ 85719, USA \\ ${ }^{11}$ Department of Physics and Astronomy, University of California, Irvine, CA 92697, USA \\ ${ }^{12}$ Space Telescope Science Institute, 3700 San Martin Drive, Baltimore, MD 21218, USA \\ Received 2013 December 1; accepted 2014 April 8; published 2014 June 11
}

\begin{abstract}
The Herschel very wide field surveys have charted hundreds of square degrees in multiple far-IR (FIR) bands. While the Sloan Digital Sky Survey (SDSS) is currently the best resource for optical counterpart identifications over such wide areas, it does not detect a large number of Herschel FIR sources and leaves their nature undetermined. As a test case, we studied seven "SDSS-invisible," very bright $250 \mu \mathrm{m}$ sources $\left(S_{250}>55 \mathrm{mJy}\right)$ in the Cosmic Assembly Near-infrared Deep Extragalactic Legacy Survey fields where we have a rich multi-wavelength data set. We took a new approach to decompose the FIR sources, using the near-IR or the optical images directly for position priors. This is an improvement over the previous decomposition efforts where the priors are from mid-IR data that still suffer from the problem of source blending. We found that in most cases the single Herschel sources are made of multiple components that are not necessarily at the same redshifts. Our decomposition succeeded in identifying and extracting their major contributors. We show that these are all ultra-luminous infrared galaxies at $z \sim 1-2$ whose high $L_{\mathrm{IR}}$ is mainly due to dust-obscured star formation. Most of them would not be selected as submillimeter galaxies. They all have complicated morphologies indicative of mergers or violent instability, and their stellar populations are heterogeneous in terms of stellar masses, ages, and formation histories. Their current ultra-luminous infrared galaxy phases are of various degrees of importance in their stellar mass assembly. Our practice provides a promising starting point for developing an automatic routine to reliably study bright Herschel sources.
\end{abstract}

Key words: galaxies: starburst - infrared: galaxies - methods: data analysis - submillimeter: galaxies

Online-only material: color figures

\section{INTRODUCTION}

In its more than four years of operation, the Herschel Space Observatory (Pilbratt et al. 2010), the largest FIR/submillimeter space telescope ever flown, produced a wealth of data awaiting exploration. It carried two imaging spectrometers, namely, the Photodetector Array Camera and Spectrometer (PACS; Poglitsch et al. 2010), observing in 100 (or 70) and $160 \mu \mathrm{m}$, and the Spectral and Photometric Imaging REceiver (SPIRE, Griffin et al. 2010), observing in 250,350 , and $500 \mu \mathrm{m}$. Together they sampled the peak of heated dust emission from $z=0$ to 6 and possibly beyond, and they have offered the best capability to date in the direct measurement of the total infrared (IR) luminosities for a large number of galaxies at $z>1$. Two of the largest Herschel extragalactic surveys, the Herschel Astrophysical Terahertz Large Area Survey (H-ATLAS; Eales et al. 2010) and the Herschel Multi-tiered Extragalactic Survey (HerMES; Oliver et al. 2012), have mapped the FIR/submillimeter universe in unprecedented detail. H-ATLAS has surveyed $\sim 570 \mathrm{deg}^{2}$

\footnotetext{
${ }^{13}$ Hubble Fellow.
}

over six areas at a uniform depth, while HerMES has observed a total of $\sim 380 \mathrm{deg}^{2}$ in several levels of depth and spatial coverage combinations ("L1" to "L7," from the deep and narrow to the wide and shallow).

The true power of these Herschel data can only be achieved when they are combined with observations at other wavelengths, most importantly in optical to near-IR (NIR) as this is the traditional regime where the stellar population of galaxies is best studied. The Sloan Digital Sky Survey (SDSS; York et al. 2000) is the most natural choice when identifying optical counterparts of the FIR sources over such large areas. However, its limited depth does not allow us to take full advantage of these already sensitive FIR data. For example, Smith et al. (2011) cross-matched the 6876 sources in the $\sim 16 \mathrm{deg}^{2} \mathrm{H}-$ ATLAS Science Demonstration Phase field to the SDSS and only $2422(35.2 \%)$ of them have reliable counterparts. While some of the identification failures are caused by the ambiguity of assigning the counterpart due to the large Herschel beam sizes, most of these FIR sources that are not matched in the SDSS are genuinely faint in the optical. This probably should not be surprising because FIR sources could be very 
dusty. Nevertheless, it is still interesting that some of the brightest Herschel sources, whose flux densities are a few tens of milliJanskys, are not visible in the SDSS images at all. The nominal $5 \sigma$ limits of the SDSS are 22.3, 23.3, 23.1, 22.3, $20.8 \mathrm{mag}$ in $u, g, r, i$, and $z$, respectively (York et al. 2000). Using a $2 \sigma$ limit, a conservative estimate of the FIR-tooptical flux density ratio of such SDSS-invisible FIR sources is $S_{\mathrm{FIR}} / S_{\text {opt }} \gtrsim 10^{4}$.

Naturally, one would speculate that these optical-faint Herschel sources are Ultra-Luminous InfraRed Galaxies (ULIRGs; see, e.g., Lonsdale et al. 2006 for a review) at high redshifts. If they are at $z \gtrsim 1$, their absence from the SDSS images can be easily explained by their large luminosity distances. If they are ULIRGs, their FIR brightness could also be understood. In this sense, the closest analogs to such objects are submillimeter galaxies (SMGs), which are usually selected at $\sim 850 \mu \mathrm{m}$ and are found to be ULIRGs at $z \sim 2-3$. On average, a typical SMG would have $850 \mu \mathrm{m}$ flux density $S_{850} \sim 5.7 \mathrm{mJy}$ and optical brightness $R \sim 24.6$ mag (see, e.g., Chapman et al. 2005). The SMGs at the faint end of the optical brightness distribution are likely at $z \sim 4-5$ or even higher redshifts (Wang et al. 2007; Capak et al. 2008; Schinnerer et al. 2008; Coppin et al. 2009; Daddi et al. 2009; Younger et al. 2009). The most extreme example is the historical HDF850.1 (Hughes et al. 1998), which has no detectable counterpart in even the deepest optical/NIR images (Cowie et al. 2009 and the references therein) and now has been confirmed to be at $z=5.183$ based on its CO lines (Walter et al. 2012). It has also been suggested that extremely dusty galaxies like HDF850.1 could play a major role in the star formation history (SFH) in the early universe (Cowie et al. 2009). If this is true, the very wide field Herschel surveys should be able to reveal a large number of such objects at very high redshifts. In fact, recently, a bright FIR galaxy discovered in the HerMES, which again has no detectable optical counterpart $(z>25.9 \mathrm{mag})$ and is only weakly visible in NIR, set a new, record-high redshift of $z=6.337$ for ULIRG (Riechers et al. 2013), approaching the end of the cosmic H I reionization epoch.

It is thus important to investigate in detail the nature of such SDSS-invisible, bright Herschel sources. Obviously, the minimum requirement to move forward is to acquire optical data that are much deeper than the SDSS. In this paper, we present our study of seven such sources that happen to be covered by the Cosmic Assembly Near-infrared Deep Extragalactic Legacy Survey (CANDELS; PIs: Faber \& Ferguson; Grogin et al. 2011; Koekemoer et al. 2011) and therefore have a rich multiwavelength data set. Our main scientific goals are to understand whether these sources are indeed high- $z$ ULIRGs and to determine the stellar populations of their host galaxies. By targeting some of the brightest Herschel sources, our study will also help address one of the most severe problems at the FIR-bright end, namely, the discrepancy between the observed bright FIR source number counts and various model predictions (see, e.g., Clements et al. 2010, and the references therein; Niemi et al. 2012). While the vast majority of other Herschel sources do not have ancillary data comparable to the data used in this current work, our study will serve as a useful guide to future investigations.

The most severe technical obstacle that we need to overcome is the long-standing source confusion (i.e., blending) problem in the FIR/submillimeter regime. The beam sizes (measured as full width at half-maximum; FWHM) of PACS are $\sim 6^{\prime \prime}-7^{\prime \prime}$ and $\sim 11^{\prime \prime}-14^{\prime \prime}$ at $70 / 100 \mu \mathrm{m}$ and $160 \mu \mathrm{m}$, respectively, depending on the scanning speeds. Similarly, the beam sizes of SPIRE are $\sim 18^{\prime \prime}, 25^{\prime \prime}$ and $36^{\prime \prime}$ at 250,350 and $500 \mu \mathrm{m}$, respectively. Therefore, source confusion can still be severe in the Herschel data, which causes ambiguity in assigning the correct counterparts. It also raises the possibility that the very high FIR flux densities of the seemingly single Herschel sources might be caused by the blending of multiple objects, each being less luminous, within a single beam. SMGs are known to suffer from exactly the same problem because of the coarse angular resolutions of the submillimeter imagers used for their discovery at single dishes. In fact, using the accurate positions determined by the submillimeter interferometry at the Submillimeter Array (SMA; Ho et al. 2004), it has been unambiguously shown that some of the brightest SMGs indeed are made of multiple objects that may or may not be physically related (Wang et al. 2011; Barger et al. 2012). Recently, a large, high-resolution $870 \mu \mathrm{m}$ interferometry survey of 126 SMGs with the Atacama Large Millimeter/submillimeter Array (ALMA) has shown that $>35 \%$ of the SMGs that originated from single-dish observations could consist of multiple objects (Hodge et al. 2013; Karim et al. 2013).

Even with the unprecedented sensitivity of the ALMA, however, it is still impractical to pin down the locations of the large number of Herschel sources and to resolve their multiplicities through submillimeter interferometry. We therefore employed an alternative, less expensive, empirical approach in this work, using deep optical/NIR data to decompose a given Herschel source and to identify its major counterpart(s) in the process. This is different from the statistical "likelihood ratio" method (Sutherland \& Saunders 1992), which would assign a counterpart based on the probabilities of all candidates but would not apportion the flux in case of multiplicity. It is also different from the de-blending approach where mid-IR data of better resolution (albeit still being coarse) would be used as the position priors (e.g., Roseboom et al. 2010; Elbaz et al. 2010; Magnelli et al. 2013). We do not take this latter approach because it could be that a prior mid-IR source is already a blend of multiple objects that are not necessarily associated. In this paper, we show that in most cases our method can successfully extract the major contributors to the FIR sources, which is sufficient if we are mainly interested in the ULIRG population with the current Herschel very wide field data. While still in its rudimentary stage, this method has the potential to be fully automated and could be critical in the Herschel fields where the "ladders" in the mid-IR are not available and can no longer be obtained due to the lack of instruments.

The paper is organized as follows. The sample and the relevant data are presented in Section 2, followed by an outline of our analyzing methods in Section 3. Due to the different data sets involved in different CANDELS fields, we present the analysis of individual objects in Sections 4, 5, and 6, respectively. We present a discussion of our results in Section 7 and summarize in Section 8. We assume the following cosmological parameters throughout: $\Omega_{M}=0.27, \Omega_{\Lambda}=0.73$, and $H_{0}=71 \mathrm{~km} \mathrm{~s}^{-1} \mathrm{Mpc}^{-1}$. The quoted magnitudes are all in the $\mathrm{AB}$ system.

\section{SAMPLE DESCRIPTION}

The SDSS-invisible, bright Herschel sources in this work were selected based on the first data release (DR1) of the HerMES team, which only includes the SPIRE data. We used their band-merged "xID" catalogs, which were constructed by fitting the point-spread function (PSF) at the source locations determined in the $250 \mu \mathrm{m}$ images (L. Wang et al., in 

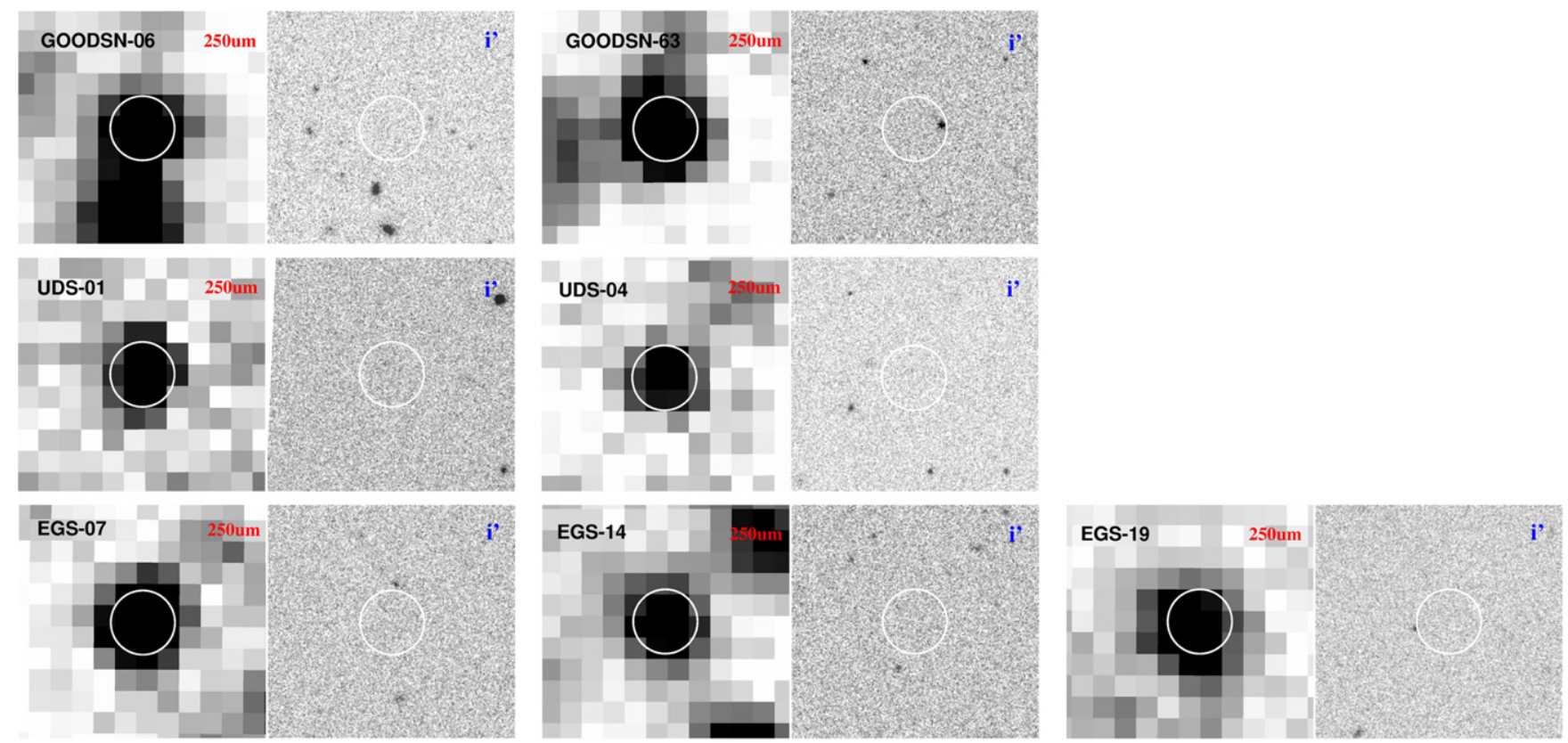

Figure 1. Image cutouts of the seven SDSS-undetected, bright Herschel sources in our sample. Two of them are in the GOODS-N field (top), two are in the UDS field (middle), and three are in the EGS field (bottom). For each source, the $250 \mu \mathrm{m}$ image is shown on the left and the SDSS $i^{\prime}$ image is shown on the right. All images are $1.2 \times 1.2$ in size. North is up and east is to the left. The white circles, which are $9^{\prime \prime}$ in radius and resemble the $250 \mu \mathrm{m}$ beam size, center on the $250 \mu \mathrm{m}$ source centroids as reported by the HerMES DR1 catalogs.

(A color version of this figure is available in the online journal.)

Table 1

Sample Summary

\begin{tabular}{llccrr}
\hline \hline HerMES ID & Short ID & R.A. and Decl. (J2000.0) & \multicolumn{1}{c}{$S_{250}$} & $S_{350}$ & $S_{500}$ \\
\hline 1HERMES S250 SF J123634.3+621241 & GOODSN06 & $12: 36: 34.3+62: 12: 41$ & $72.1 \pm 5.4$ & $44.4 \pm 3.2$ & $17.3 \pm 3.7$ \\
1HERMES S250 SF J123730.9+621259 & GOODSN63 & $12: 37: 30.9+62: 12: 59$ & $55.7 \pm 5.4$ & $59.9 \pm 3.1$ & $46.3 \pm 3.5$ \\
1HERMES S250 SF J021806.0-051247 & UDS01 & $02: 18: 06.0-05: 12: 47$ & $55.2 \pm 9.7$ & $40.0 \pm 7.6$ & $15.3 \pm 6.1$ \\
1HERMES S250 SF J021731.1-050711 & UDS04 & $02: 17: 31.1-05: 07: 11$ & $67.6 \pm 9.7$ & $49.0 \pm 7.7$ & $12.2 \pm 6.3$ \\
1HERMES S250 SF J141900.3+524948 & EGS07 & $14: 19: 00.3+52: 49: 48$ & $81.2 \pm 6.0$ & $71.7 \pm 4.9$ & $42.9 \pm 5.2$ \\
1HERMES S250 SF J142025.9+525935 & EGS14 & $14: 20: 25.9+52: 59: 35$ & $57.4 \pm 6.0$ & $45.6 \pm 4.9$ & $22.3 \pm 6.0$ \\
1HERMES S250 SF J141943.4+525857 & EGS19 & $14: 19: 43.4+52: 58: 57$ & $57.4 \pm 6.0$ & $24.4 \pm 5.4$ & $9.2 \pm 7.1$ \\
\hline
\end{tabular}

Notes. The listed flux densities in $250\left(S_{250}\right), 350\left(S_{350}\right)$, and $500 \mu \mathrm{m}\left(S_{500}\right)$ are in mJy. These values are taken from the HerMES DR1 catalogs, which are based on the "xID" catalogs (L. Wang et al., in preparation). The errors include the confusion noise. "Short ID" is a nickname assigned here for simplicity.

preparation). These catalogs are cut at a bright flux density level of $55 \mathrm{mJy}$ for 250 and $350 \mu \mathrm{m}$, and $30 \mathrm{mJy}$ for $500 \mu \mathrm{m}$.

Among these released HerMES fields, six of them have SDSS coverage, three of which have overlap with the CANDELS fields. These three fields are the "L2_GOODS-N" $\left(\sim 0.59 \mathrm{deg}^{2}\right.$, 53 sources in the HerMES DR1 catalog), "L3_Groth-Strip" ( $\sim 1.15 \mathrm{deg}^{2}, 74$ sources), and "L6_XMM-LSS-SWIRE" fields ( $\sim 22.58 \mathrm{deg}^{2}, 2320$ sources), which cover the CANDELS "GOODS-N," "EGS" and "UDS" fields, respectively. ${ }^{14}$ The CANDELS Hubble Space Telescope (HST) data, taken by the Advanced Camera for Surveys (ACS) and/or the IR channel of the Wide Field Camera 3 (WFC3), include 5 (in GOODS-N), 13 (in Extended Groth Strip, EGS) and 4 (in Ultra Deep Survey (UDS)) of these sources. We examined them in the SDSS DR9 images and selected those that do not have any optical detections in any of the five bands within 6 " (approximately $\sim 3 \times$ of the

14 "GOODS-N" stands for the northern field of the Great Observatories Origins Deep Survey (Giavalisco et al. 2003), "EGS" stands for the extended Groth Strip, and "UDS" stands for the Ultra-Deep Survey component of the United Kingdom Infrared Telescope (UKIRT) Infrared Deep Sky Survey (UKIDSS). positional accuracy) to the reported $250 \mu \mathrm{m}$ source centroids. This resulted in 2, 3, and 2 sources in GOODS-N, EGS, and UDS, respectively, which form the sample studied by this work. Table 1 lists the photometry of these seven sources from the HerMES public catalogs, and Figure 1 shows their images in the SPIRE $250 \mu \mathrm{m}$ and the SDSS $i^{\prime}$ bands. In addition to the HerMES three-band SPIRE data, the sources in L2_GOODS-N and L3_Groth-Strip also have the PACS 100 and $160 \mu \mathrm{m}$ data from the DR1 of the PACS Evolutionary Probe program (PEP; Lutz et al. 2011).

The three CANDELS fields have a wide range of ancillary data, and those used in the spectral energy distribution (SED) analysis and/or morphological study are listed below.

1. GOODS-N. HST ACS F435W (hereafter $B_{435}$ ), F606W $\left(V_{606}\right)$, F775W $\left(i_{775}\right)$, and F850LP $\left(z_{850}\right)$ from the GOODS program; F098M $\left(Y_{098}\right), \mathrm{F} 105 \mathrm{~W}\left(J_{105}\right)$, and F160W $\left(H_{160}\right)$ from the CANDELS program; Spitzer InfraRed Array Camera (IRAC) 3.6, 4.5, 5.8, and $8.0 \mu \mathrm{m}$, and Multiband Imaging Photometer for Spitzer (MIPS) $24 \mu \mathrm{m}$ from the GOODS program; MIPS $70 \mu \mathrm{m}$ from the Far-Infrared Deep 
Extragalactic Legacy survey (FIDEL; M. Dickinson et al., in preparation); and ground-based deep $U$-band image taken by the GOODS team at the KPNO $4 \mathrm{~m}$ MOSAIC (M. Dickinson 2006, private communication).

2. UDS. ACS $V_{606}$ and F814W $\left(I_{814}\right)$, WFC3 IR $J_{105}$, and $H_{160}$ from the CANDELS program; IRAC 3.6 and $4.5 \mu \mathrm{m}$ from the Spitzer Extended Deep Survey (SEDS; PI: Fazio; Ashby et al. 2013); MIPS $24 \mu \mathrm{m}$ from the Spitzer UDS program (SpUDS; PI: Dunlop); ground-based $u^{*}, g^{\prime}, r^{\prime}, i^{\prime}$, and $z^{\prime}$ data from the final data release ("T0007") of the CFHT Legacy Survey Wide component (CFHTLS-Wide); and the ground-based $J, H$, and $K_{s}$ data from the UDS component of the United Kingdom Infrared Telescope (UKIRT) Deep Sky Survey (UKIDSS).

3. EGS. ACS $V_{606}$ and $I_{814}$ from the All-wavelength Extended Groth strip International Survey (AEGIS; Davis et al. 2007); WFC3 IR $J_{105}$ and $H_{160}$ from the CANDELS program; ground-based $u^{*}, g^{\prime}, r^{\prime}, i^{\prime}$, and $z^{\prime}$ data from the final release of the CFHTLS Deep component (CFHTLS-Deep); MIPS 24 and $70 \mu \mathrm{m}$ from the FIDEL program; IRAC 3.6 and $4.5 \mu \mathrm{m}$ that incorporate the data from both the SEDS program and the Spitzer Guaranteed Time Observing program 8 (Barmby et al. 2008).

In addition, public $\mathrm{X}$-ray and high-resolution radio images and/or catalogs are also available in these fields, which improve the interpretation of our sources. The GOODS-N has the Chandra 2 Ms data (Alexander et al. 2003) and the Very Large Array (VLA) $1.4 \mathrm{GHz}$ data (Morrison et al. 2010), the UDS field has the X-ray source catalog based on the XMM-Newton observations (Ueda et al. 2008) and the VLA $1.4 \mathrm{GHz}$ source catalog (Simpson et al. 2006), and the EGS field has the Chandra 800 ks data from the AEGIS program (K. Nandra et al., in preparation; Laird et al. 2009) and the VLA 1.4 GHz data (Ivison et al. 2007; Willner et al. 2012).

\section{OVERVIEW OF METHODS FOR ANALYSIS}

As the deep optical-to-NIR data reveal, there are always multiple candidate counterparts for any $250 \mu \mathrm{m}$ source in our sample. Our basic goal is to decompose the blended objects to determine the major contributors to the FIR emissions and to reveal their nature by analyzing their optical-to-far-IR SEDs. Here we briefly describe our methods.

\subsection{Source Decomposition and Flux Calibration}

We used the GALFIT package developed by Peng et al. (2002) to do the decomposition. While its wide usage by the community is mostly to study galaxy morphologies, GALFIT has a straightforward capability of fitting the PSF at multiple, fixed locations. This well suits our cases because all the potential components are effectively point sources at the angular resolutions of the Herschel instruments. Although other PSF fitting software tailored for crowded field photometry (such as ALLSTAR in DAOPHOT; Stetson 1987) could also be used in the decomposition of Herschel data (e.g., Rawle et al. 2012), we chose GALFIT because it produced the best results in our tests. When the source signal-to-noise ratio $(\mathrm{S} / \mathrm{N})$ is sufficient, GALFIT is capable of differentiating the offset of the input source position as small as $\sim 1 / 10$ of a native pixel (C. Y. Peng 2013 , private communication). This corresponds to $\sim 2^{\prime \prime}$ in the $250 \mu \mathrm{m}$ band, and is comparable to the astrometric accuracy (i.e., the accuracy of their centroids) of our bright $250 \mu \mathrm{m}$ sources.
As we are interested in constructing the FIR SEDs, we decomposed not only the $250 \mu \mathrm{m}$ but also the PACS 160 and $100 \mu \mathrm{m}$ images when they were available. We also extended the same decomposition to the MIPS 70 and $24 \mu \mathrm{m}$ data because the point-source assumption still largely holds at the MIPS resolution. On the other hand, we did not attempt to decompose the SPIRE 350 and $500 \mu \mathrm{m}$ data because the beam sizes of these two bands are so large that it is difficult to achieve reliable results using our current approach. We generated the $250 \mu \mathrm{m}$ PSF by using a symmetric 2D Gaussian of 18". 15 FWHM, which has already been proved to be a good fit to the point sources in the HerMES images (see, e.g., Roseboom et al. 2010; Smith et al. 2011). For the PACS images, we adopted the green (for $100 \mu \mathrm{m}$ ) and red (for $160 \mu \mathrm{m}$ ) PSFs provided by the PEP team along with their DR1, which were built by stacking a set of bright, isolated, and point-like sources. The MIPS 70 and $24 \mu \mathrm{m}$ PSFs were created using the daophot.psf task in IRAF. For each field and band, a PSF was constructed from a set of 7 to 15 bright and isolated sources, which are all point sources as judged from the WFC3 $H_{160}$ image.

For a given $250 \mu \mathrm{m}$ source, we identified its potential components by searching for objects in the high-resolution near-IR or optical images within $r=18^{\prime \prime}$ of the $250 \mu \mathrm{m}$ centroid. In other words, the diameter of the searching area is twice the FWHM of the $250 \mu \mathrm{m}$ beam. The positions of the potential optical/near-IR components were then used as the priors for GALFIT to extract the fluxes at these locations in various bands. This is different from using the $24 \mu \mathrm{m}$ source positions as the priors, and we did not take the latter approach because, as mentioned in Section 1 , it is not uncommon that a single $24 \mu \mathrm{m}$ source is actually a blended product of multiple objects that are not necessarily related. In addition, using optical/near-IR position priors has the advantage of being able to directly tie the FIR source to the ultimate counterpart(s). Our first choice for the detection image was the WFC $3 H_{160}$ image and the second choice was the ACS $I_{814}$ image if $H_{160}$ was not available. The third choice was the ground-based $i$-band image if it was more appropriate than the HST images (for instance, to avoid splitting one galaxy of complex morphology into several sub-components at the HST resolution). Regardless of the exact choice, the search always resulted in several tens of objects. Currently, it is not desirable for our routine to deal with such a large number of objects because there are still a large number of intermediate steps that require human intervention (see below). As only a fraction of these objects actually contribute to the FIR emission, in this current work we chose to use the PACS 160 and $100 \mu \mathrm{m}$ and the MIPS $24 \mu \mathrm{m}$ images to narrow down the input list for GALFIT. The general guideline was that only the objects that could have non-negligible $24 \mu \mathrm{m}$ emissions should be further considered. While we could have developed some quantitative criteria for this process, we opted to simply rely on visual inspection because this was only an interim step that we are planning to replace in the near future. For this work, we were conservative in eliminating objects from the input list, and included those that are at the outskirts of the $24 \mu \mathrm{m}$ source footprints.

The above procedure narrowed the input lists to $\lesssim 10$ objects each. Similar to the philosophy of PSF fitting in crowded stellar fields (e.g., Stetson 1987), one should seek to fit simultaneously the exact objects that are contributing flux because this is when the most accurate result can be obtained. The implementation was rather difficult, however. In our case, fitting all the objects from the input list simultaneously was often not satisfactory. The symptoms were bad residual images after subtracting the fitted 
objects, large errors associated with the decomposed fluxes, or in some cases, the crashing of the decomposition process. Therefore, we took two complementary approaches.

In the first approach, which we dub as the "automatically iterative" approach, all the potential components were still fitted simultaneously, however, those with derived fluxes smaller than the associated flux errors were deemed negligible and removed from the input list for the next round. The simultaneous fit was repeated using the cleaned input list until no negligible objects were left. This procedure usually converged after 2-3 iterations. Among the surviving components, some could have fitted fluxes an order of magnitude smaller than the others. When this happened, a new fit was performed using an input list that contained only the major components. If the residual image was of the same quality (judged by $\chi^{2}$ and also visual inspection) as in the previous round, these major components were deemed as the only contributors and those less important ones were ignored. Otherwise we kept the results from the previous round. Finally, a "sanity check" was carried out on the surviving objects by fitting them one at a time. Usually it was obvious that there were residuals left at the locations of the other components, indicating that more than one component contributed significantly to the source and hence a simultaneous fit was necessary. However, in some cases, the fit to only one object produced a clean residual image of the same quality as the one from the simultaneous fit to multiple objects, and when this happened, we flagged this source as being a degenerate case and offered alternative interpretations.

The other approach, which we call the "trial-and-error" approach, was highly interactive. This method had to be used when the automatically iterative method failed at the first step: it either crashed or resulted in unreasonable survivors with very large errors that could not be improved by removing any of the survivors (i.e., the iteration failed). In this case, we started from our best guess of the most likely counterpart and fit for only this object in the first round. We then checked the residual map to see if there were any residuals left at the positions of any other objects in the original input list. If yes, these objects were added to the fitting list, one at a time, and the fit iterated until reaching the best result possible. The iteration stopped when adding more objects either produced only negligible contributors or started to produce unreasonable results.

We note that these two approaches can be integrated and further improved in their implementation. In particular, it is possible to not only automate the trial-and-error approach but also use it to narrow down the input list without the reference to additional data such as the MIPS $24 \mu \mathrm{m}$ image. While it is beyond the scope of this paper to fully develop this automatic routine, we demonstrate its feasibility in the Appendix. We also note that we did not use the radio source positions as the priors. This is because some objects that have non-negligible contributions to the FIR emissions could fall below the sensitivity of the currently available radio data.

The PSF-fitting fluxes obtained in the procedures above should be corrected for the finite PSF sizes, which could be achieved by comparing our PSF-fitting results to the curve-ofgrowth aperture photometry on a set of isolated point sources. In addition, the PACS images also suffer from light lost caused by the application of the high-pass filtering in the reduction process, which is not compensated for in the currently released images and thus should also be corrected. To simply the process, we did the following for this work. For the PACS data, we derived the total correction factors for 100 and $160 \mu \mathrm{m}$ bands in each field by comparing our PSF-fitting results against those in the PEP DR1 single-band catalogs. For the SPIRE data, we adopted the photometry reported in the HerMES DR 1 catalog as the total flux densities and proportionated among the multiple contributors according to our decomposition results.

To obtain realistic uncertainties in the Herschel bands, we carried out extensive simulations. For each source, we simulated it by adding the PSFs according to the final decomposition result, put the simulated object in several hundreds of random locations on the real image, ran the decomposition at these locations, and obtained a distribution of the recovered fluxes. The dispersion of this distribution was adopted as the instrumental errors. The errors due to the confusion noise were then added in quadrature to the instrumental errors to obtain the final errors. For PACS 100 and $160 \mu \mathrm{m}$, we adopted the confusion noise values of 0.15 and $0.68 \mathrm{mJy}^{\text {beam }^{-1}}$ from Magnelli et al. (2013), and for the SPIRE data we inherited the values from the HerMES DR1 catalogs, which are 5.8, 6.3, and $6.8 \mathrm{mJy}^{\text {beam }}{ }^{-1}$ based on Nguyen et al. (2010). For our objects, the confusion noise only contributes insignificantly to the total error in the PACS bands, however, it dominates the total error in the SPIRE bands. For the sake of consistency, the errors in the MIPS 24 and $70 \mu \mathrm{m}$ were obtained in the same way but without the confusion term.

As mentioned above, we did not apply the decomposition to the 350 and the $500 \mu \mathrm{m}$ data where the resolutions are much worse and the source $\mathrm{S} / \mathrm{N}$ is generally much lower than at the $250 \mu \mathrm{m}$. This means that generally these two bands cannot be incorporated in our analysis. However, in a few cases we find that the vast majority of the $250 \mu \mathrm{m}$ flux is from only one object (others contribute a few percent at most), or that the major contributors are likely at the same redshift and thus we can discuss their combined properties. For such sources, we directly use the 350 and the $500 \mu \mathrm{m}$ measurements from the HerMES DR1 catalogs in our study.

\subsection{SED Fitting}

In order to understand the objects under question, their redshifts are needed. While some have spectroscopic redshifts, most of our objects have to rely on photometric redshift $\left(z_{\mathrm{ph}}\right)$ estimates. To derive $z_{\mathrm{ph}}$, we used the SEDs that extend from the optical to the IRAC wavelengths (to $8.0 \mu \mathrm{m}$ when possible). The inclusion of the IRAC data was important but often nontrivial. As many of our sources have input objects that are close to each other, they are usually blended in the IRAC images and thus need to be decomposed as well. For most objects, the pointsource assumption no longer holds in IRAC. Therefore, we used the TFIT technique (Laidler et al. 2006) for this purpose, where the image templates were constructed from the $H_{160}$ images, and then were convolved by the IRAC PSFs to fit the IRAC images.

We used the Hyperz software (Bolzonella et al. 2000) and the stellar population synthesis (SPS) models of Bruzual \& Charlot (2003; hereafter BC03) to estimate $z_{\mathrm{ph}}$. The latest implementation of Hyperz also includes the tool to derive other physical quantities such as the stellar mass $\left(M^{*}\right)$, the age $(T)$, etc. ("hyperz_mass"; M. Bolzonella 2012, private communication) from the models. We adopted the BC03 models of solar metallicity and the initial mass function (IMF) of Chabrier (2003) and used a series of exponentially declining SFHs with $\tau$ ranging from 1 Myr to $20 \mathrm{Gyr}$. The simple stellar population (SSP) model, which was treated as $\tau=0$, was also included. The models were allowed to be reddened by dust following Calzetti's law (Calzetti 2001), with $A_{V}$ ranging from zero to 4 mag. 
The above SED fitting procedure does not consider the effect of emission lines. To assess how this might impact the derived physical properties, we also used the LePhare software (Arnouts et al. 1999 and Ilbert et al. 2006) to analyze the same SEDs. LePhare can calculate the strengths of the common emission lines based on the star formation rate (SFR) of the underlying SPS models, which we chose to be the same sets of models used in the Hyperz analysis. The results derived by LePhare in most cases are very close to those derived using Hyperz, and thus we only include the discussion of the Hyperz results in this paper.

A key quantity in revealing the nature of our sources is the total IR luminosity, $L_{\mathrm{IR}}$, traditionally calculated over rest-frame $8-1000 \mu \mathrm{m}$. To derive $L_{\mathrm{IR}}$, we used our own software tools to fit the mid-to-far-IR SED to the starburst models of Siebenmorgen \& Krügel (2007; hereafter SK07) at either the spectroscopic redshift, when available, or the adopted $z_{\mathrm{ph}}$ from above. We adopted their "9 kpc" and "15 kpc" models, which SK07 produces for the starbursts at high redshifts. When possible, we also derive the dust temperature $\left(T_{d}\right)$ and mass $\left(M_{d}\right)$, using the code from Casey (2012), which fits the FIR SED to a modified blackbody spectrum combined with a power law extending from the mid-IR. As we only have limited passbands, we fixed the slope of the mid-IR power law to $\alpha=2.0$ and the emissivity of the blackbody spectrum to $\beta=1.5$ (Chapman et al. 2005; Pope et al. 2006; Casey et al. 2009). This approach usually does not produce a good fit at $24 \mu \mathrm{m}$, presumably due to the fixed $\alpha$. Therefore, we ignored the $24 \mu \mathrm{m}$ data points when deriving $T_{d}$ and $M_{d}$. The code derives two different dust temperatures, one being the best-fit temperature of the graybody $\left(T_{d}^{\mathrm{fit}}\right)$ and the other being the temperature according to Wien's displacement law which corresponds to the peak of the emission as determined by the best-fit graybody $\left(T_{d}^{W}\right)$. We adopted $T_{d}^{\text {fit }}$. Finally, we also obtained the gas mass, $M_{\text {gas }}$, by applying the nominal Milky Way gas-to-dust-mass ratio of 140 (e.g., Draine et al. 2007), with the caveat that this ratio could strongly depend on the metallicities (e.g., Draine et al. 2007; Galametz et al. 2011; Leroy et al. 2011).

Another important quantity is the SFR. SED fitting in the optical-to-NIR regime provides the SFR intrinsic to the SFH of the best-fit $\mathrm{BC} 03$ model (hereafter $\mathrm{SFR}_{\mathrm{fit}}$ ), which naturally takes into account the effect of dust extinction. However, there could still be star formation processes completely hidden by dust, which would not be counted by SFR $_{\text {fit }}$ and could only be estimated through the measurement of $L_{\mathrm{IR}}$. A common practice is to exercise the conversion given by Kennicutt (1998), which is $\mathrm{SFR}_{\mathrm{IR}}=1.0 \times 10^{-10} L_{\mathrm{IR}}$ after adjusting for a Chabrier IMF (see, e.g., Riechers et al. 2013). ${ }^{15}$ This conversion assumes solar metallicity and is valid for a starbursting galaxy that has a constant SFR over 10-100 Myr and whose dust re-radiates all of the bolometric luminosity. The total SFR would then be some combination of $\mathrm{SFR}_{\mathrm{fit}}$ and $\mathrm{SFR}_{\mathrm{IR}}$. However, this should not be a straightforward sum of the two. The reason for this is that our measured $L_{\mathrm{IR}}$ includes not only the IR emission from the region completely blocked by dust (hereafter $L_{\mathrm{IR}}^{\mathrm{blk}}$ ) but also the contribution from the exposed region where a fraction of its light is extincted by dust and is re-radiated in the FIR (hereafter $L_{\mathrm{IR}}^{\mathrm{ext}}$ ), i.e., $L_{\mathrm{IR}}=L_{\mathrm{IR}}^{\mathrm{blk}}+L_{\mathrm{IR}}^{\mathrm{ext}}$. In other words, SFR $\mathrm{IR}_{\mathrm{IR}}$ derived by applying Kennicutt's conversion directly to $L_{\mathrm{IR}}$ would have already included part of the contribution from SFR $\mathrm{fit}_{\text {. }}$. To deal with this problem, we calculated $L_{\mathrm{IR}}^{\mathrm{ext}}$ by integrating the

\footnotetext{
${ }^{15}$ Using the conversion for a Salpeter IMF, the SFR will be a factor of 1.7 higher.
}

difference between the reddened and the de-reddened spectra from the best-fit BC03 model and assuming that this amount of light is completely re-radiated in the FIR. We then obtained $L_{\mathrm{IR}}^{\mathrm{blk}}=L_{\mathrm{IR}}-L_{\mathrm{IR}}^{\mathrm{ext}}$, and calculated $\mathrm{SFR}_{\mathrm{IR}}^{\mathrm{blk}}=1.0 \times 10^{-10} L_{\mathrm{IR}}^{\mathrm{blk}}$, where SFR $\mathrm{IR}_{\mathrm{I}}^{\mathrm{bl}}$ is the "net" SFR in the region completely blocked by dust.

From the derived SFR and $M^{*}$, one can calculate the specific $\mathrm{SFR}$ as SSFR $=\mathrm{SFR}_{\mathrm{tot}} / M^{*}$. The caveat here is that the stellar mass derived by SED fitting as mentioned above is only for the relatively exposed region and does not include the completely obscured region. Nevertheless, we can still use a related but more appropriate quantity in this context, namely, the stellar mass doubling time $\left(T_{\mathrm{db}}\right)$, which is defined as the time interval necessary to further assemble the same amount of stellar mass of the exposed region should the galaxy keeps its SFR constant into the future. Specifically, we can obtain $T_{\mathrm{db}}^{\mathrm{tot}}=M^{*} / \mathrm{SFR}_{\mathrm{tot}}$ and $T_{\mathrm{db}}^{\mathrm{blk}}=M^{*} / \mathrm{SFR}_{\mathrm{IR}}^{\mathrm{blk}}$. Comparing the latter quantity to the age $(T)$ of the existing stellar population in the exposed region is particularly interesting, as this is a measure of the importance of the completely dust-blocked region in the future evolution of the galaxy.

Finally, the measurement of $L_{\mathrm{IR}}$ enables us to examine the well-known FIR-radio relation (see Condon 1992 for review) for the sources that have radio data. We used the conventional formalism in Helou et al. (1985), which takes the form of

$$
\begin{aligned}
q_{\mathrm{IR}}= & \log \left[\left(S_{\mathrm{IR}} / 3.75\right.\right. \\
& \left.\left.\times 10^{12} \mathrm{~W} \mathrm{~m}^{-2} \mathrm{~Hz}^{-1}\right) /\left(S_{1.4 \mathrm{GHz}}^{0} / \mathrm{W} \mathrm{m}^{-2} \mathrm{~Hz}^{-1}\right)\right],
\end{aligned}
$$

where $S_{\mathrm{IR}}$ is the integrated IR flux while $S_{1.4 \mathrm{GHz}}^{0}$ is the radio flux density at the rest-frame $1.4 \mathrm{GHz}$. For the k-correction in radio, we assume $S_{v} \propto v^{-0.8}$. The original usage of $S_{\mathrm{IR}}$ (the quantity "FIR" in Helou et al. 1985) is defined between the rest-frame 42.5 and $122.5 \mu \mathrm{m}$. In order to take full advantage of the Herschel spectral coverage, we opted to adopt $S_{\mathrm{IR}}$ as in Ivison et al. (2010), where this quantity is defined from 8 to $1000 \mu \mathrm{m}$ in the restframe. In practice, we calculated $S_{\text {IR }}$ using the best-fit SK07 model. For reference, the mean value that Ivison et al. (2010) obtain using Herschel sources in the GOODS-N is $q_{\mathrm{IR}}=2.40 \pm 0.24$.

\section{SOURCES IN THE GOODS-N}

\subsection{GOODSN06 (J123634.3+621241)}

This source is in the SMG sample of Wang et al. (2004; their GOODS 850-19), however, it was detected at the $<4 \sigma$ level $\left(S_{850}=3.26 \pm 0.85 \mathrm{mJy}\right)$ and was not included in the SMA observations of Barger et al. (2012).

\subsubsection{Morphologies and Potential Components}

Figure 2 shows this source in various bands from $250 \mu \mathrm{m}$ to $H_{160}$. Within an $18^{\prime \prime}$ radius, there are 74 objects in $H_{160}$ that have $\mathrm{S} / \mathrm{N} \geqslant 5$ (measured in the MAG_AUTO aperture). By inspecting the FIR and the $24 \mu \mathrm{m}$ images, it is obvious that only six of these objects could possibly contribute to GOODSN06. In order of the distances between their centroids to the $250 \mu \mathrm{m}$ position, these potential components are marked alphabetically from "A" to "F." Objects A, B, and C, which are 1".38, 2".50, and 3 ". 15 , away from the $250 \mu \mathrm{m}$ position, respectively, are completely blended in the $24 \mu \mathrm{m}$ image as a single source. This is the brightest $24 \mu \mathrm{m}$ source within $18^{\prime \prime}\left(S_{24}=446 \pm 5 \mu \mathrm{Jy}\right)$, and also dominates the flux in $70 \mu \mathrm{m}$. The centroid of this single 

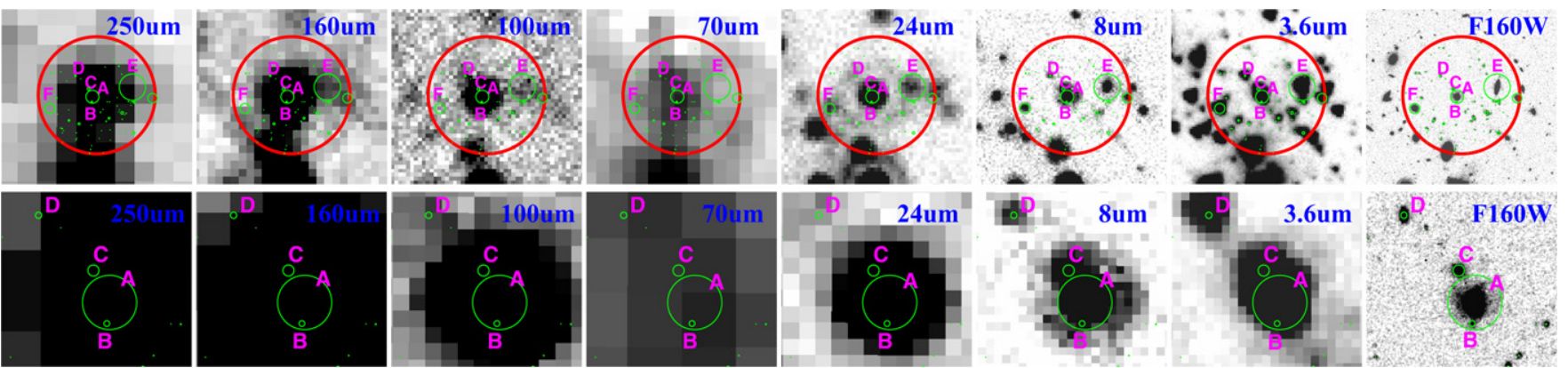

Figure 2. Vicinity of GOODSN06 (top) and zoomed-in view of its possible contributors (bottom) in SPIRE $250 \mu \mathrm{m}$, PACS 160 and $100 \mu \mathrm{m}$, MIPS 70 and $24 \mu \mathrm{m}$, IRAC 8 and $3.6 \mu \mathrm{m}$, and WFC $3 H_{160}$. North is up and east is to the left. The red circles have $36^{\prime \prime}$ diameter, twice the beam FWHM at $250 \mu \mathrm{m}$. The objects identified in $H_{160}$ within this radius are marked by the green circles whose sizes are proportional to their $H_{160}$ brightnesses. The possible contributors to the $250 \mu \mathrm{m}$ emission are labeled by the letters in purple.

(A color version of this figure is available in the online journal.)
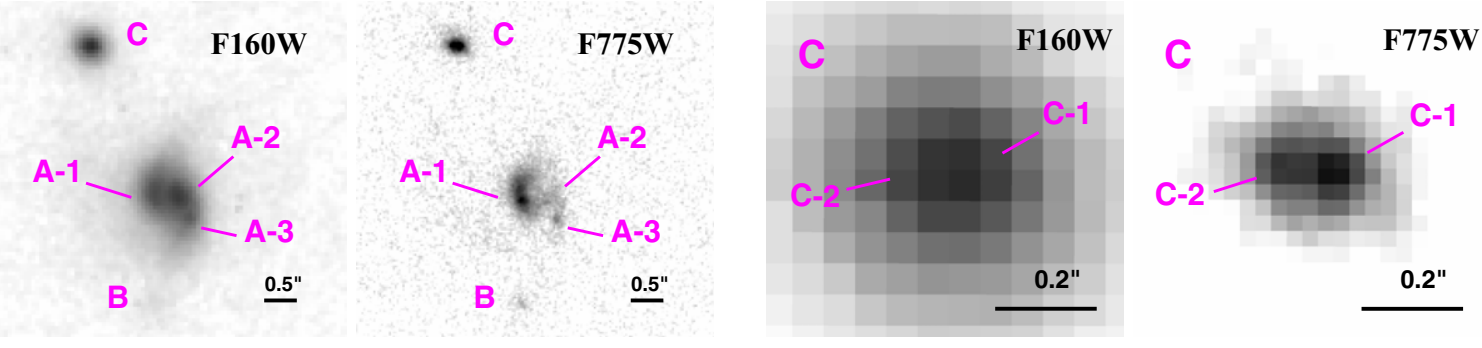

Figure 3. Detailed morphologies of A (left) and C (right) in $H_{160}$ and $i_{775}$. The sub-components are labeled numerically. A-2 is prominent in NIR but is very weak in optical, indicating that it is obscured by dust. $\mathrm{C}$ shows double nuclei in the higher resolution $i_{775}$ image.

(A color version of this figure is available in the online journal.)

$24 \mu \mathrm{m}$ source is also coincident with the centroid of the emission in 100,160 , and $250 \mu \mathrm{m}$. In the $8.0 \mu \mathrm{m}$ image, $\mathrm{A}$ and $\mathrm{C}$ are separated and both are well detected, and hence it is reasonable to assume that they both contribute to the $24 \mu \mathrm{m}$ flux. B is still blended with $\mathrm{A}$ in the $8.0 \mu \mathrm{m}$ image, and we cannot rule it out as a contributor to the $24 \mu \mathrm{m}$ flux. Under our assumption, this means that these three objects could all be contributors to the FIR emission.

As it turns out, A and $\mathrm{C}$ have spectroscopic redshifts of 1.224 and 1.225, respectively (Barger et al. 2008). Their separation is 2 ". 64 , which corresponds to $22.1 \mathrm{kpc}$ at these redshifts, and is well within the scale of galaxy groups. This suggests that they could indeed be associated. The high-resolution HST images reveal that both $\mathrm{A}$ and $\mathrm{C}$ have complicated morphologies and that B could be part of A. This is show in Figure 3. While it is difficult to be separated photometrically, $A$ is actually made of three sub-components, which we label as "A-1," "A-2," and "A-3" (Figure 3, left). The ACS images show that A-1 has a compact core and a one-sided, curved tail. A-2 is invisible in the ACS optical images, but it is the most prominent of the three in the WFC3 IR and also has a compact core. A-3 is similar to A-1 in the WFC3 IR but extends to the opposite direction and is much fainter. The one-sided, curved tail of $\mathrm{A}-3$ is invisible in the ACS optical. The core of A-2 has nearly the same angular separation from those of $A-1$ and $A-3$, which is about 0 '.38, or $3.2 \mathrm{kpc}$ at $z=1.22$. Therefore, $\mathrm{A}$ is likely a merging system. In fact, B could well be part of this system, as it seems to connect with the tail of $A-3$. C looks smooth and regular in the WFC3 images, however, in the higher resolution ACS images it shows two nuclei, which is also indicative of a merging process (Figure 3, right). All this suggests that it will be reasonable to consider the $\mathrm{A} / \mathrm{B} / \mathrm{C}$ complex as a single system when treating the FIR emission.
While it is only marginally detected in $24 \mu \mathrm{m}$, component $\mathrm{D}$ seems to have non-negligible fluxes in both 70 and $100 \mu \mathrm{m}$. This object is invisible in $B_{435}$ and $V_{505}$, and is marginally detected in $i_{775}$, but starts to be very prominent in $z_{850}$ and in the WFC3 IR and the IRAC bands. Its morphology in $z_{850}$ is rather irregular and shows two sub-components in $Y_{105}$ and $J_{125}$. However, in $H_{160}$ it looks like a normal disk galaxy. While all this suggests that it could be a dusty galaxy and thus could have non-negligible FIR emission, its position is 8."88 offset from the $250 \mu \mathrm{m}$ centroid, and thus, it is less likely a major contributor to the $250 \mu \mathrm{m}$ flux.

Finally, E and F cannot be associated with any of the above because they have spectroscopic redshifts of 0.562 and 0.9617 , respectively (Barger et al. 2008). While both of them are prominent sources in $24 \mu \mathrm{m}$, only $\mathrm{E}$ is well visible in $160 \mu \mathrm{m}$ and thus could be a significant contributor to the FIR emission.

\subsubsection{Optical-to-near-IR SED Analysis}

The optical-to-near-IR SED analysis for objects A to D follows the procedure outlined in Section 3.2. The SED was constructed based on the photometry in the ACS, the WFC3 IR, and the IRAC bands. The ACS and the WFC3 photometry were obtained by running the SExtractor software (Bertin \& Arnouts 1996) in dual-image mode on the set of images that are registered and PSF-matched to the $H_{160}$ band. The $H_{160}$ image was used as the detection image, and the colors were measured in the MAG_ISO apertures. The $H_{160}$ MAG_AUTO magnitudes are then adopted as the reference to convert the colors to the magnitudes that go into the SED. The IRAC magnitudes are the TFIT results using the $H_{160}$ image as the morphological template and the PSF derived from the point sources in the field.

The results are summarized in Figure 4, which includes $z_{\mathrm{ph}}$ and the physical properties of the underlying stellar populations 

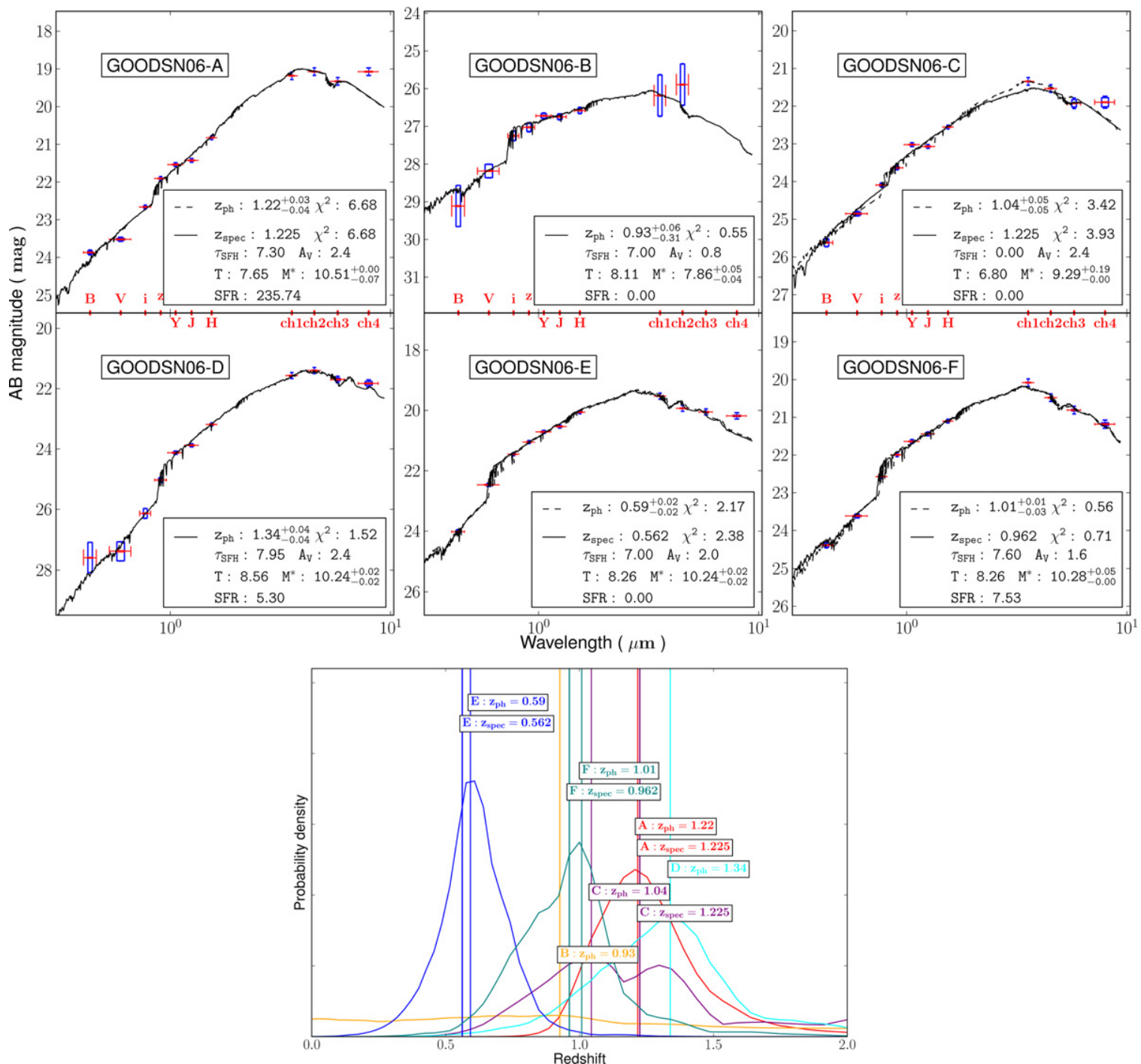

Figure 4. Optical-to-NIR SED analysis of the possible contributors to GOODSN06. The top panel summarizes the SED fitting results of these objects individually. The data points are shown together with the best-fit $\mathrm{BC} 03$ models. For the objects that have $z_{\text {spec }}$, the solid curves are the best-fit model at this redshift, while the dashed curves are the best-fit model when $z$ is a free parameter. The most relevant physical properties inferred from the models are summarized in the boxed region: reduced $\chi^{2}, \tau$ (in Gyr), $A_{V}, T$ ( $\log T$ in year), $M *\left(\log M^{*}\right.$ in $M_{\odot}$ ), and $\mathrm{SFR}_{\mathrm{fit}}\left(\right.$ in $M_{\odot} \mathrm{yr}^{-1}$ ). The bottom panel shows the probability distribution functions of the photometric redshifts $(P(z))$ of these objects, which are distinguished by different colors. The best-fit $z_{\mathrm{ph}}$ and the $z_{\mathrm{spec}}$ values (when available) are shown in boxes and are also marked by the vertical lines.

(A color version of this figure is available in the online journal.)

such as the stellar mass $\left(M^{*}\right)$, the extinction $A_{V}$, the age $(T)$, the characteristic star-forming timescale $(\tau)$, and the dust-corrected SFR. This figure also shows $P(z)$, which is the probability density function of $z_{\mathrm{ph}}$ for all these objects. The available $z_{\mathrm{spec}}$ agrees with our $z_{\text {ph }}$ reasonably well. The largest discrepancy happens in $\mathrm{C}$, which has $\Delta z=0.185$, or $\Delta z /(1+z)=0.08$. This discrepancy is explained by the secondary $P(z)$ peak at $z_{\mathrm{ph}} \sim 1.2$. Object $\mathrm{B}$, a very close neighbor to $\mathrm{A}$, has $z_{\mathrm{ph}}=0.93$, which may seem to suggest that it is not related to $A$. However, its $P(z)$ is rather flat over all redshifts and does not have any distinct peak, and therefore its $z_{\mathrm{ph}}$ cannot be used as strong evidence to argue for or against its relation with A. From these results and the morphologies, it is reasonable to believe that $B$ is only a less important satellite to A. Object D has $z_{\mathrm{ph}}=1.34 \pm 0.04$, and is different from $z_{\text {spec }}$ of the $\mathrm{A} / \mathrm{C}$ complex by $\Delta z /(1+z)=0.05$. This is within the accuracy of our $z_{\mathrm{ph}}$ technique, suggesting that D could be in the same group. Nevertheless, in the following analysis we still treat it separately.

\subsubsection{Decomposition in Mid-to-far-IR}

The decomposition of GOODSN06 was done in the SPIRE $250 \mu \mathrm{m}$, the PACS 160 and $100 \mu \mathrm{m}$, and the MIPS 70 and $24 \mu \mathrm{m}$. This source has two neighbors to the south ("S1" and "S2"), which, while being outside of $r=18$ ", could still contaminate the target in the 250,160 , and $70 \mu \mathrm{m}$ bands where the beam sizes are large. While in other cases we only fit the objects within $r=18^{\prime \prime}$, for this source we must consider these two contaminators that are farther out. We adopted their positions in $H_{160}$, as there is no ambiguity in their identifications, and fit them together with all the components of GOODSN06 when decomposing in these three bands. They are well separated from the target in 100 and $24 \mu \mathrm{m}$, and hence did not enter the decomposition process in these two bands.

For illustration, Figure 5 shows the decomposition in $250 \mu \mathrm{m}$. The simultaneous fit to all five objects within $r=18^{\prime \prime}$ (A to E) resulted in B and C as the only two survivors. However, GALFIT 

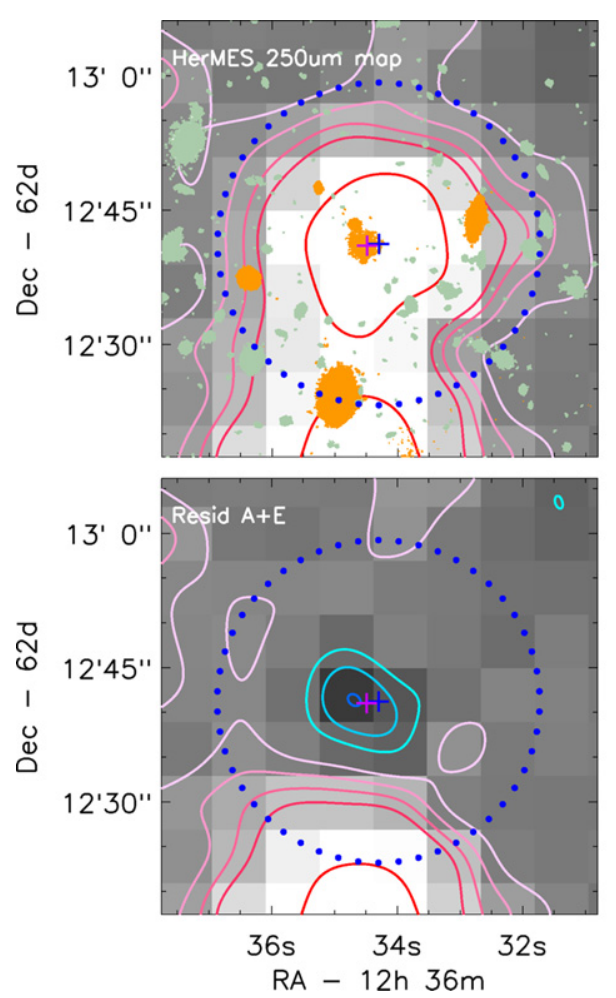
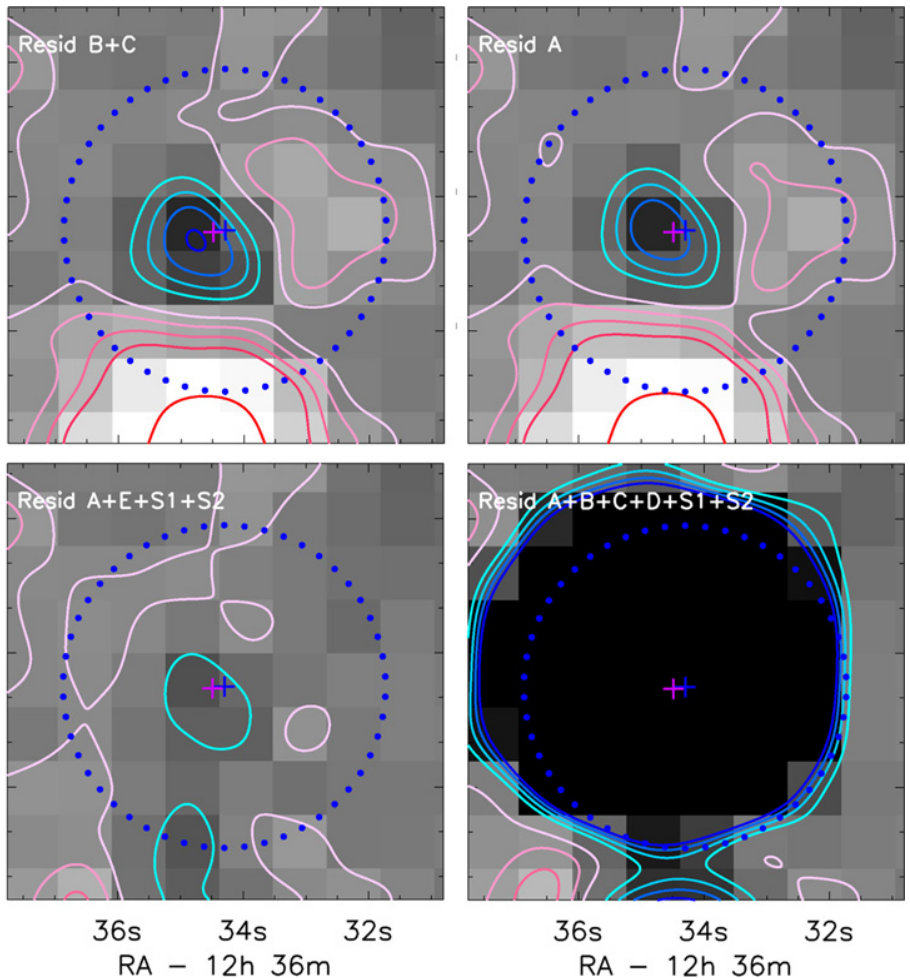

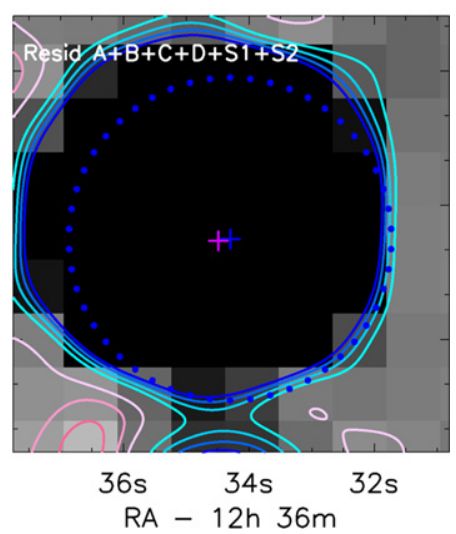

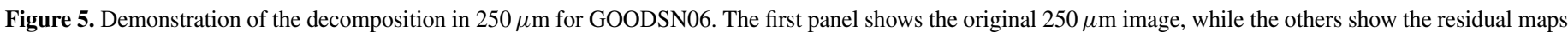

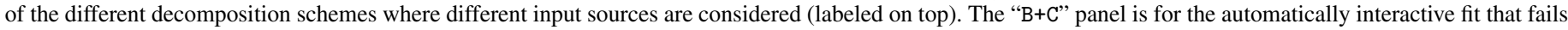

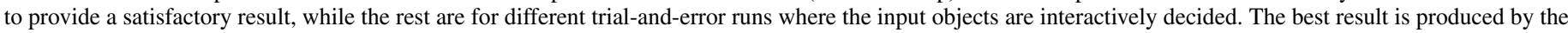

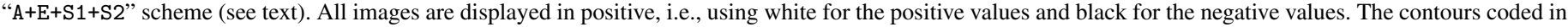

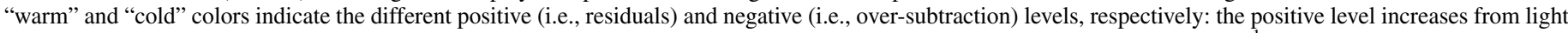

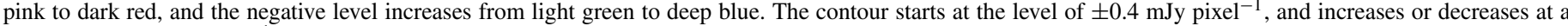

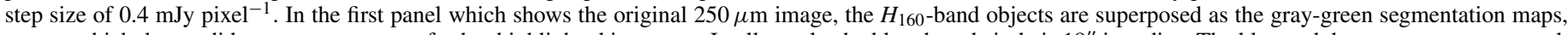

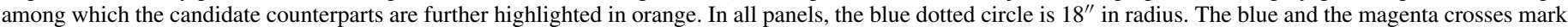
the $250 \mu \mathrm{m}$ source centroid and the VLA $1.4 \mathrm{GHz}$ source position, respectively.

(A color version of this figure is available in the online journal.)

reported very large flux errors for these two objects, and their extracted fluxes would have very low GALFIT fitting $\mathrm{S} / \mathrm{Ns}(<3$ and $<0.5$, respectively). Removing either of the two did not improve the result. Therefore, we had to use the trial-and-error approach. Judging from the mid-IR images (Section 4.1.1, in particular the $24 \mu \mathrm{m}$ image) and from the optical-to-NIR SED analysis (Section 4.1.2), it is reasonable to conclude that A, B, and $C$ are associated, with $A$ being by far the most dominant. The forced fit for A only, however, showed a severe oversubtraction and yet a clear residual at the location of E. The forced fit at A and E significantly improved; however, the extraction at A still had a large error. When we included the two southern neighbors (S1 and S2) and fit them together with $\mathrm{A}$ and $\mathrm{E}$, the error of $\mathrm{A}$ was greatly reduced. If we left out $\mathrm{E}$, the residual persistently showed up at this location, indicating that the contamination from $\mathrm{S} 1$ and $\mathrm{S} 2$ is not the reason and that the inclusion of $\mathrm{E}$ is necessary. The fit with A, E, S1, and S2 seems to be the best among all possibilities and forcing the fit to additional objects would create completely non-physical results. Therefore, we adopted the $\mathrm{A}+\mathrm{E}+\mathrm{S} 1+\mathrm{S} 2$ scheme, which concludes that A contributes $82 \%$ of the total flux to GOODSN06 and E contributes the other $18 \%$.

The automatically iterative decomposition procedure was successful in other bands. In $160 \mu \mathrm{m}$, it converged on $\mathrm{A}+\mathrm{E}$, and introducing $\mathrm{S} 1$ further reduced the errors and improved the residuals. In $100 \mu \mathrm{m}$, it converged on $\mathrm{A}+\mathrm{D}+\mathrm{E}$ and did not need to include the southern neighbors. In $70 \mu \mathrm{m}$, it converged on $\mathrm{A}+\mathrm{E}+\mathrm{F}$, and including both $\mathrm{S} 1$ and $\mathrm{S} 2$ further improved the results. In $24 \mu \mathrm{m}$, it converged on $\mathrm{A}+\mathrm{E}+\mathrm{F}$. In all these bands, $\mathrm{A}$ is the most dominant object as well.

The flux densities of the major component A based on the above decomposition results are summarized in Table 2. The VLA 1.4 GHz data from Morrison et al. (2010), which have the positional accuracy of $0^{\prime \prime} .02-00^{\prime} 03$, reveal a source with $S_{1.4 \mathrm{GHz}}=0.201 \pm 0.010 \mathrm{mJy}$ at R.A. $=12^{\mathrm{h}} 36^{\mathrm{m}} 34^{\mathrm{s}} .49$, Decl. $=62^{\circ} 12^{\prime} 41^{\prime \prime} .0$, and is right at the location of A-2. A is also a moderate X-ray source in Alexander et al. (2003), which has a rest-frame $0.5-2 \mathrm{keV}$ luminosity of $L_{0.5-2}=8.1 \times 10^{41} \mathrm{erg} \mathrm{s}^{-1}$ (Barger et al. 2008), and hence most likely is powered by stars rather than an active galactic nucleus (AGN; see, e.g., Georgakakis et al. 2007).

\subsubsection{Total IR Emission and Stellar Populations}

Figure 6 shows our fit to the mid-to-far-IR SED, which results in $L_{\mathrm{IR}}=4.0 \times 10^{12} L_{\odot}$ at $z_{\mathrm{spec}}=1.225$. This is in the ULIRG regime. Based on the optical-to-near-IR SED fitting in Section 4.1.2, we integrated the difference between the reddened, best-fit model template spectrum of $\mathrm{A}$ and its de-reddened version (see Section 3.2), and obtained $L_{\mathrm{IR}}^{\mathrm{ext}}=2.3 \times 10^{12} L_{\odot}$. The "net" IR luminosity from the region completely blocked by dust is therefore $L_{\mathrm{IR}}^{\mathrm{blk}}=1.7 \times 10^{12} L_{\odot}$, which implies $\mathrm{SFR}_{\mathrm{IR}}^{\mathrm{blk}}=170 M_{\odot} \mathrm{yr}^{-1}$. The best-fit $\mathrm{BC} 03$ model for $\mathrm{A}$ in Section 4.1.2 gives $\mathrm{SFR}_{\mathrm{fit}}=236 M_{\odot} \mathrm{yr}^{-1}$. Thus $\mathrm{SFR}_{\mathrm{tot}}=406 M_{\odot} \mathrm{yr}^{-1}$. While the $\mathrm{A} / \mathrm{B} / \mathrm{C}$ complex is 

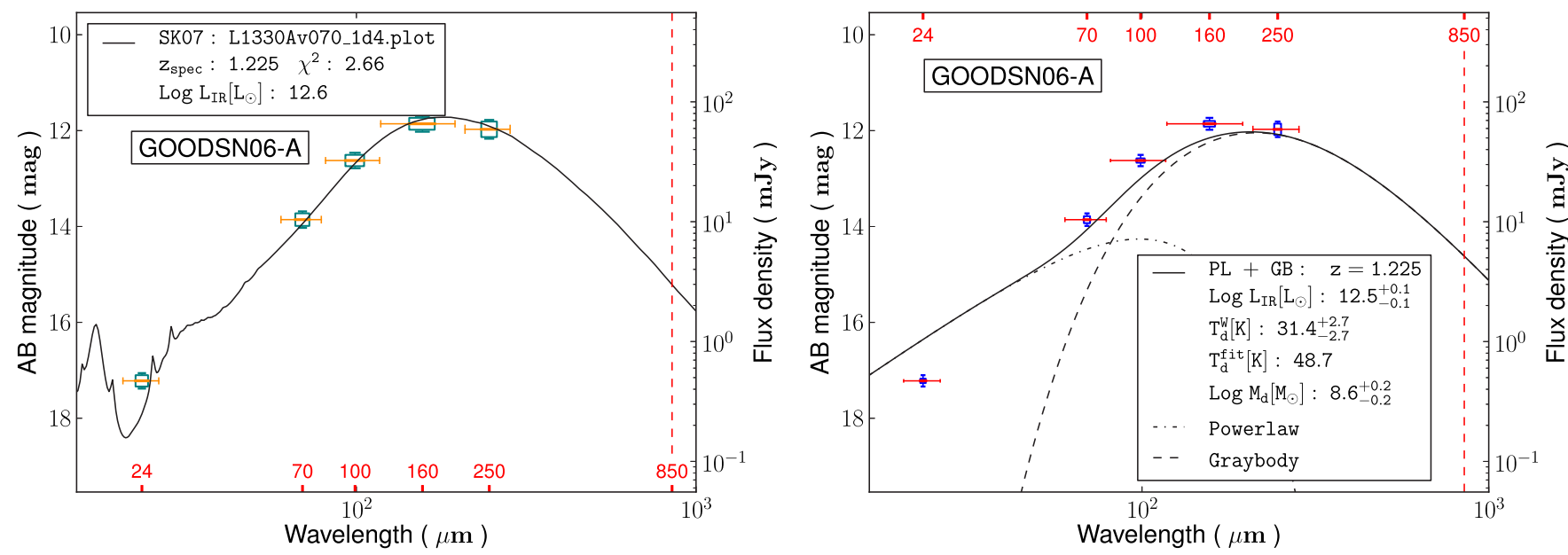

Figure 6. Mid-to-FIR SED fitting of GOODSN06-A at $z_{\mathrm{spec}}=1.225$. The relevant parameters derived from the fits are summarized in the boxed regions. The left panel shows the fit to the SK07 models to derive $L_{\mathrm{IR}}$, where the green boxes are the data points and the superposed curve is the best-fit model. The right panel shows the fit to derive the dust temperature and the dust mass, where the analytic models are made of a power law and a graybody (see Section 3.2). The data points are shown in blue. The best-fit model is shown as the black solid curve, while its power-law and graybody components are shown as the dot-dashed and the dashed curves, respectively. In both panels, the most relevant best-fit parameters are summarized in the boxes. The red vertical dashed lines are at $850 \mu \mathrm{m}$, which is the wavelength where SMGs are selected.

(A color version of this figure is available in the online journal.)

Table 2

Decomposed Mid-to-far-IR Fluxes of the Major Components

\begin{tabular}{|c|c|c|c|c|c|c|c|c|}
\hline ID & R.A. and Decl. (J2000.0) & $S_{24}$ & $S_{70}$ & $S_{100}$ & $S_{160}$ & $S_{250}$ & $S_{350}$ & $S_{500}$ \\
\hline GOODSN06-A & $12: 36: 34.519+62: 12: 40.99$ & $0.473 \pm 0.023$ & $10.40 \pm 0.70$ & $32.4 \pm 1.4$ & $65.6 \pm 3.6$ & $59.0 \pm 6.5$ & $\ldots$ & . \\
\hline GOODSN63-A & $12: 37: 30.767+62: 12: 58.74$ & $0.170 \pm 0.009$ & $\ldots$ & $5.0 \pm 0.5$ & $22.4 \pm 1.1$ & $55.7 \pm 5.4$ & $59.9 \pm 3.1$ & $46.3 \pm 3.5$ \\
\hline UDS01-B & $02: 18: 06.114-05: 12: 50.11$ & $0.319 \pm 0.020$ & $\ldots$ & $\ldots$ & $\ldots$ & $32.6 \pm 9.5$ & $\ldots$ & $\ldots$ \\
\hline UDS01-D & $02: 18: 06.159-05: 12: 44.93$ & $0.465 \pm 0.020$ & $\ldots$ & $\ldots$ & $\ldots$ & $22.6 \pm 9.5$ & $\ldots$ & $\ldots$ \\
\hline (UDS01-BD) & & $0.784 \pm 0.025$ & $\ldots$ & $\ldots$ & $\ldots$ & $55.2 \pm 9.7$ & $40.0 \pm 7.6$ & $15.3 \pm 6.1$ \\
\hline UDS04-A & 02:17:31.159-05:07:09.15 & $0.670 \pm 0.020$ & $\ldots$ & $\ldots$ & $\ldots$ & $67.7 \pm 9.7$ & $49.0 \pm 7.7$ & $12.2 \pm 6.3$ \\
\hline EGS07-1 & $14: 19: 00.202+52: 49: 47.74$ & $0.620 \pm 0.015$ & $1.96 \pm 0.50$ & $13.9 \pm 1.0$ & $36.8 \pm 2.4$ & $64.3 \pm 6.5$ & $\ldots$ & $\ldots$ \\
\hline EGS14-5 & $14: 20: 25.704+52: 59: 31.75$ & $0.273 \pm 0.015$ & $\ldots$ & $8.0 \pm 1.3$ & $28.2 \pm 3.2$ & $29.2 \pm 6.2$ & $\ldots$ & $\ldots$ \\
\hline EGS14-9 & $14: 20: 26.424+52: 59: 39.33$ & $0.243 \pm 0.017$ & $\ldots$ & $7.8 \pm 1.6$ & $14.7 \pm 3.1$ & $28.2 \pm 6.1$ & $\ldots$ & $\ldots$ \\
\hline EGS19-A-1 & $14: 19: 43.436+52: 58: 58.19$ & $0.170 \pm 0.010$ & $\ldots$ & $7.7 \pm 1.5$ & $25.8 \pm 2.1$ & $29.3 \pm 6.1$ & $\ldots$ & $\ldots$ \\
\hline EGS19-A-2 & $14: 19: 43.583+52: 58: 59.02$ & & & & & & & \\
\hline EGS19-A-4 & $14: 19: 43.470+52: 58: 52.20$ & & & & & & & \\
\hline
\end{tabular}

Notes. The mid-to-far-IR flux densities (in mJy) of the major components of the Herschel sources in Table 1, based on our decomposition results. For completeness, we also include the case of UDS01-BD where the two components UDS01-B and D are added together. For EGS19, the listed fluxes are for the major component EGS19-A', which is a "composite source" made of EGS19-A-1, A-2, and A-4. For GOODSN06, GOODSN63, UDS01, the positions reported here are based on the $H_{160}$ images. For UDS04, EGS07, EGS14, and EGS19, the reported positions are based on the CFHTLS images.

very likely an interacting system, it is interesting to note that the ULIRG phenomenon only happens to A, and that B and C are not triggered. Furthermore, it seems that the current star formation activities all concentrate on $\mathrm{A}$. The best-fit model for $\mathrm{C}$ is an SSP, which formally has no ongoing star formation. It is plausible that A itself is an ongoing merger (judging from its complex morphology), and hence is undergoing an intense starburst, while its companion $\mathrm{C}$ and the smaller satellite $\mathrm{B}$ are still on the way falling to it.

The stellar mass of this system is also dominated by A, for which we obtained $3.2 \times 10^{10} M_{\odot}$ (Section 4.1.2). The stellar mass of $\mathrm{C}$ is one order of magnitude less at $1.9 \times 10^{9} M_{\odot}$ and is negligible. Therefore, this implies SSFR $=12.7 \mathrm{Gyr}^{-1}$. Using the stellar mass doubling timescales as discussed in Section 3.2, we obtained $T_{\mathrm{db}}^{\mathrm{tot}}=79 \mathrm{Myr}$ and $T_{\mathrm{db}}^{\mathrm{blk}}=188 \mathrm{Myr}$, respectively. Interestingly, the optical-to-near-IR SED fitting for A in Section 4.1.2 gives an age of $T=45 \mathrm{Myr}$. Its SFH has $\tau=20 \mathrm{Myr}$, which is comparable to this best-fit age. In other words, the SFH of the exposed region is a rather short burst, and this every young galaxy has been in starbursting mode ever since its birth, although it is unclear whether it could be an ULIRG in the earlier time. While the star formation in the completely dust-blocked region is playing a less important role as compared to that in the exposed region $\left(T_{\mathrm{db}}^{\mathrm{blk}}\right.$ is more than a factor of four longer than $T$ ), it will still be significant should the gas reservoir suffice (see below).

The fit for the dust temperature and the dust mass is shown Figure 6. $T_{d}^{\mathrm{fit}}=48.7 \mathrm{~K}$ is on the high side of the usual dust temperatures of the SMGs, which are around $\sim 30-40 \mathrm{~K}$ (e.g., Chapman et al. 2005, 2010; Pope et al. 2006; Magnelli et al. 2010, 2012; Swinbank et al. 2014). In fact, as mentioned earlier, GOODSN06 is only a marginal SMG and has $S_{850}=$ $3.26 \pm 0.85 \mathrm{mJy}$. This is a demonstration that the Herschel bands are less biased against ULIRGs of high dust temperature. From the best-fit SK07 model, one would predict $S_{850}=2.29 \mathrm{mJy}$, in agreement with the actual measurement to $1.14 \sigma$. The dust mass we obtained is $M_{d}=3.7 \times 10^{8} M_{\odot}$. If assuming the nominal gas-to-dust ratio of $\sim 140$, the gas reservoir is $5.2 \times 10^{10} M_{\odot}$, 

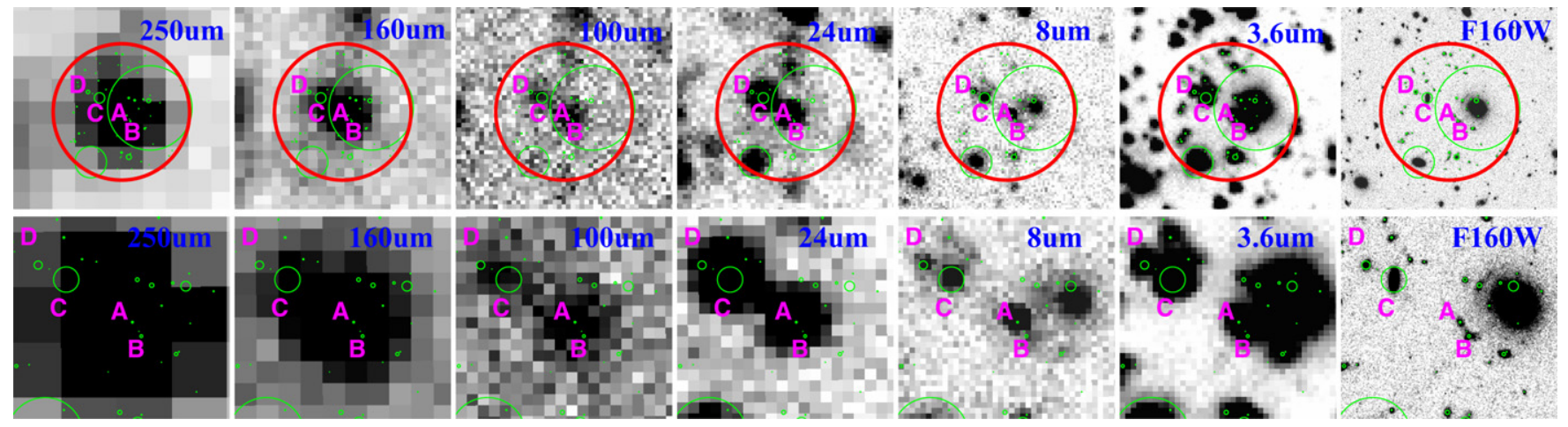

Figure 7. FIR to near-IR images of GOODSN63. The organization and the legends are the same as in Figure 2 . This source does not have MIPS $70 \mu \mathrm{m}$ data. The possible contributors to the $250 \mathrm{~m}$ emission are labeled by the letters in purple.

(A color version of this figure is available in the online journal.)
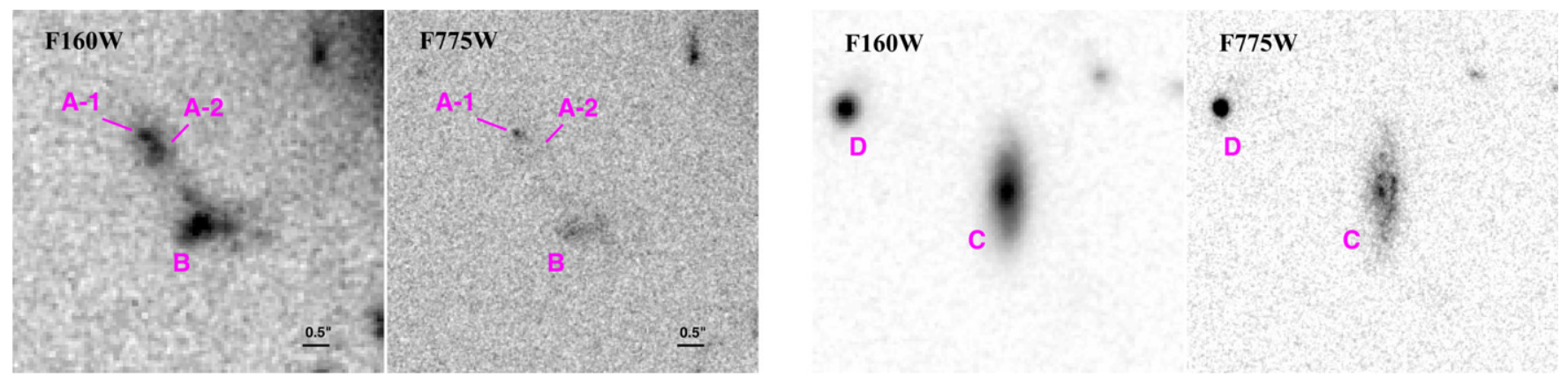

Figure 8. NIR and optical morphologies of the possible contributors to GOODSN63, shown in $H_{160}$ and $i_{775}$. The left panel is for A and B, and the right panel is for C and D. A clearly breaks up into two sub-components in $H_{160}$, labeled as "A-1" and "A-2," the latter of which is only barely visible in the optical.

(A color version of this figure is available in the online journal.)

sufficient for the whole system to continue for another $\sim 130 \mathrm{Myr}$ or the dust-blocked region for $\sim 300$ Myr.

Finally, we examine the FIR-radio relation using the formalisms summarized in Section 3.2, for which we obtained $q_{\mathrm{IR}}=2.44$, which is in good agreement with the mean value of $2.40 \pm 0.24$ of Ivison et al. (2010).

\subsection{GOODSN63 (GOODSN-J123730.9+621259)}

This source has a multi-wavelength data set nearly as rich as GOODSN06 does except that it lacks $70 \mu \mathrm{m}$ image. While being a single source in $250 \mu \mathrm{m}$, it breaks up into two sources (but still blended) in 160 and $100 \mu \mathrm{m}$. According to the PEP DR1 catalog, these two PACS components have $S_{160}=22.8 \pm 1.4$ and $9.5 \pm 1.2 \mathrm{mJy}$, and $S_{100}=4.9 \pm 0.4$ and $3.6 \pm 0.4 \mathrm{mJy}$, respectively. This source is a previously discovered SMG (Wang et al. 2004, their GOODS 850-6), whose position has been precisely determined by the SMA observations to be R.A. $=12^{\mathrm{h}} 37^{\mathrm{m}} 30 \mathrm{~s} .80$, Decl. $=62^{\circ} 12^{\prime} 59^{\prime \prime} .00(\mathrm{~J} 2000)$, and has $S_{850}=14.9 \pm 0.9 \mathrm{mJy}$ (Barger et al. 2012).

\subsubsection{Morphologies and Potential Components}

Figure 7 shows the images of GOODSN63. Within $18^{\prime \prime}$ radius, there are 63 objects in $H_{160}$ with $\mathrm{S} / \mathrm{N} \geqslant 5$. The inspection shows that only four of them could be significant contributors in the FIR. These four objects, marked alphabetically as "A" to D," are $1^{\prime \prime} .68,3$.'40, 6". 86 , and $10^{\prime \prime} .27$ away from the $250 \mu \mathrm{m}$ position, respectively. In $24 \mu \mathrm{m}, \mathrm{A}$ and $\mathrm{B}$ are blended as a single source, while $\mathrm{C}$ and $\mathrm{D}$ are blended as another. These are the two brightest $24 \mu \mathrm{m}$ sources within $r=18^{\prime \prime}$, and their positions are consistent with the two local maxima in 160 and $100 \mu \mathrm{m}$. The brightest object in $H_{160}$ (marked by the largest green circle in Figure 7) has a spectroscopic redshift of $z=0.5121$ (Barger et al. 2008) and is not visible in $24 \mu \mathrm{m}$ at all, and hence it is not likely to have any significant FIR emission.

Objects A and B show complex morphologies. The WFC3 IR images reveal that A consists of two subcomponents, A-1 and A-2, which are only 0.'30 apart (Figure 8, left). A-2 is completely invisible in all the ACS bands. In the IR images, B shows a dominant core that is irregular in shape and also has irregular, satellite components to its northwestern side. In the ACS images, its core is less distinct and the satellite components are invisible. All this suggests that both A and B are likely very dusty systems and that both could be going through violent merging process. While the small separation between them (1".80) suggests that they could be an interacting pair, our SED analysis below shows that they actually are not associated.

Objects C and D are 3".42 apart. They have spectroscopic redshifts of 0.9370 and 1.3585, respectively (Barger et al. 2008), and therefore are not associated. The morphologies of these two objects are shown in Figure 8 (right) in details. While it is a rather smooth disk galaxy in the rest-frame optical, $\mathrm{C}$ shows knotty structures and dust lanes in rest-frame UV, suggestive of active star formation. On the other hand, D appears as a smooth spheroid in the rest-frame UV to optical. All this suggests that $\mathrm{C}$ is the major contributor to the $24 \mu \mathrm{m}$ flux, which is consistent with the $24 \mu \mathrm{m}$ morphology as the $24 \mu \mathrm{m}$ centroid is closer to the position of $\mathrm{C}$ than to $\mathrm{D}$.

\subsubsection{Optical-to-near-IR SED Analysis}

Figure 9 summarizes the results of the optical-to-near-IR SED analysis for the four potential components. The SEDs used here 

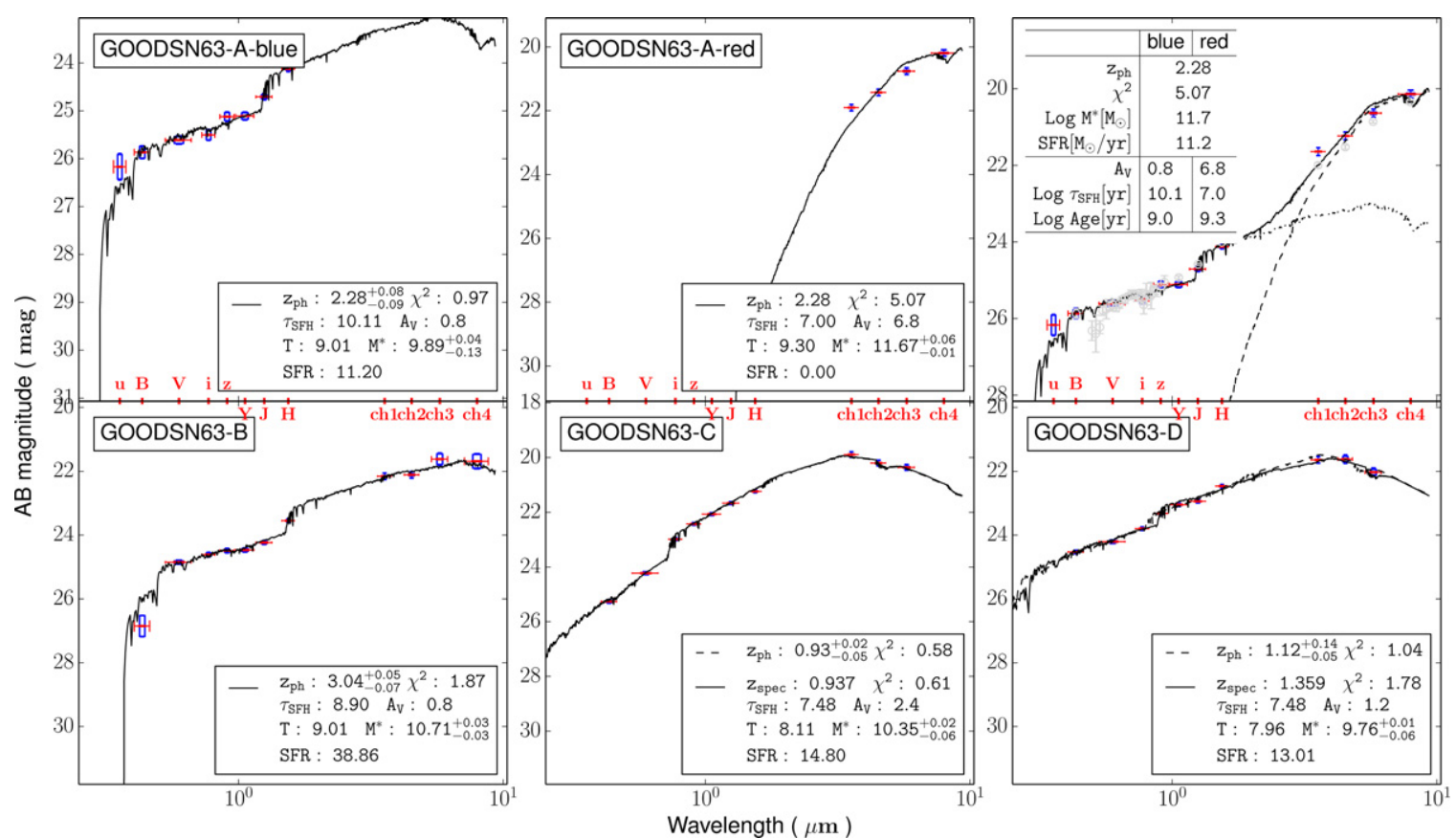

Figure 9. Optical-to-NIR SED analysis of the possible contributors to GOODSN63. Legends are the same as in Figure 4. The three panels on the top are all for object A: from left to right, these are the fit to its blue sub-component, its red sub-component, and the sum of the two. The gray data points in the upper-right panel are from the Rainbow database. Note that the "blue" and the "red" refer to the SED sub-components but not the morphological sub-components A-1 and A-2. See the text for details.

(A color version of this figure is available in the online journal.)

are based on the same photometry as in Section 4.1.2, except for A where it is augmented by the $U$-band image.

The fitting of $\mathrm{C}$ gives $z_{\mathrm{ph}}=0.93$, in excellent agreement with its $z_{\text {spec }}=0.937$. D has $z_{\text {ph }}=1.22$ as opposed to $z_{\text {spec }}=1.359$, nevertheless they are still in reasonable agreement $\left(\Delta_{z} /(1+z)=\right.$ 0.06). A and B do not have spectroscopic redshifts. B is not detected in the $U$-band image, and would be selected as a Lyman break galaxy at $z \approx 3$. In fact, the fit to its SED, either with or without including the $U$-band constraint, gives $z_{\mathrm{ph}}=3.04$.

A deserves some more detailed discussion. The singlecomponent fit as used for other objects fails to explain the SED of A. We have also tested a different set of SFHs where the SFR increases with time (Papovich et al. 2011), however, A still cannot be fitted by a single component. Briefly, the strong IRAC fluxes and the large break between WFC3 IR and IRAC tend to assign a high redshift of $z_{\mathrm{ph}} \gtrsim 3$ to the object, which would then give an implausibly high stellar mass of $>10^{12} M_{\odot}$. On the other hand, the optical fluxes (especially the detection in the $U$-band) are only consistent with significantly lower redshifts. Therefore, we attempted to fit this object with two components. We took a two-step approach. We first fit the blue part of the SED up to $H_{160}$, using the whole set of $\mathrm{BC} 03$ models as usual. We obtained the best fit at $z_{\mathrm{ph}}=2.28$. The best-fit model is of moderate stellar mass $\left(7.8 \times 10^{9} M_{\odot}\right)$ and age $(1.0 \mathrm{Gyr})$, and has a very extended SFH $(\tau=13 \mathrm{Gyr})$ with the current $\mathrm{SFR}_{\mathrm{fit}}=11.2 \mathrm{M}_{\odot} \mathrm{yr}^{-1}$. This best-fit template, which extends to the IRAC wavelengths, was then subtracted from the original SED to produce the SED for the red part. This red part remains very strong in the IRAC bands, as the blue component only contributes a small amount at these wavelengths. It has nearly zero flux from $U$ to $J_{125}$, but still has non-zero residual flux in $H_{160}$. We then fitted this red SED, from $H_{160}$ to $8.0 \mu \mathrm{m}$, at the fixed redshift of 2.28 from the blue component. We also relaxed the constraint on the dust extinction and allowed $A_{V}$ to vary from 0 to $10 \mathrm{mag}$. We indeed found a reasonable best-fit model, which is a highly extincted $\left(A_{V}=6.8 \mathrm{mag}\right)$, high stellar mass $\left(4.7 \times 10^{11} M_{\odot}\right)$, old (age $2.0 \mathrm{Gyr}$ ) burst ( $\tau=10 \mathrm{Myr}$ ). The result of this two-step fit is summarized again in Figure 9 with more details. While this is probably not the unique solution, it offers a plausible explanation for the SED of A. In addition, we also examined if the red part could be explained by a power-law AGN in the form of $f_{v} \propto v^{-\alpha}$. The commonly adopted slope is $\alpha=1.0-1.3$ in the rest-frame near-IR (i.e., the IRAC bands in the observer's frame for this object), and changes to a flatter value of $\alpha=0.7-0.8$ when extending into the rest-frame optical regime (see, e.g., Elvis et al. 1994; Richards et al. 2006; Assef et al. 2010). Such power laws, however, cannot provide any reasonable fit to the data. We found that the IRAC data points, with or without the extension to the MIPS $24 \mu \mathrm{m}$, could only be explained by a very steep slope of $\alpha=1.8-1.9$. Adopting this slope in the restframe optical and ignoring the possible flattening of the SED, we would get still get $H_{160} \sim 23.6 \mathrm{mag}$, which is much higher with the requirement of $H_{160}=28.8$ mag based on the SED of the red part. In fact, this is also significantly brighter than the observed total of $H_{160}=24.12$ mag. While we cannot definitely rule out the possibility that there might be an embedded AGN in GOODSN63-A, there is no compelling evidence that such an AGN component exists. Based on our analysis, we believe that the optical-to-near-IR emission of GOODSN63-A is dominated by stellar populations.

Interestingly, Barger et al. (2012) derive $z_{\mathrm{ph}}=2.7$ for GOODSN63 based on the radio-to-submillimeter flux ratio. To further check on the redshift of this GOODSN63-A, we used the "Rainbow" database in the GOODS-N, which incorporates the 24 narrow-band images $(0.50-0.95 \mu \mathrm{m})$ from the Survey of High- $z$ Absorption Red and Dead Sources (SHARDS) program (Pérez-González et al. 2013) and the GOODS \& CANDELS ACS/WFC/IRAC data as used above (see the gray data points 

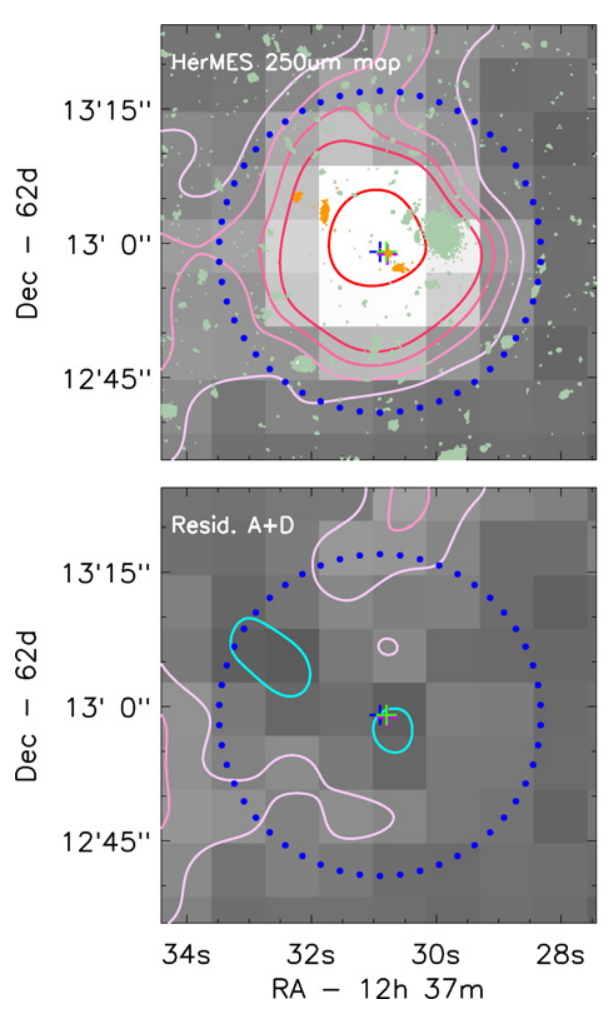
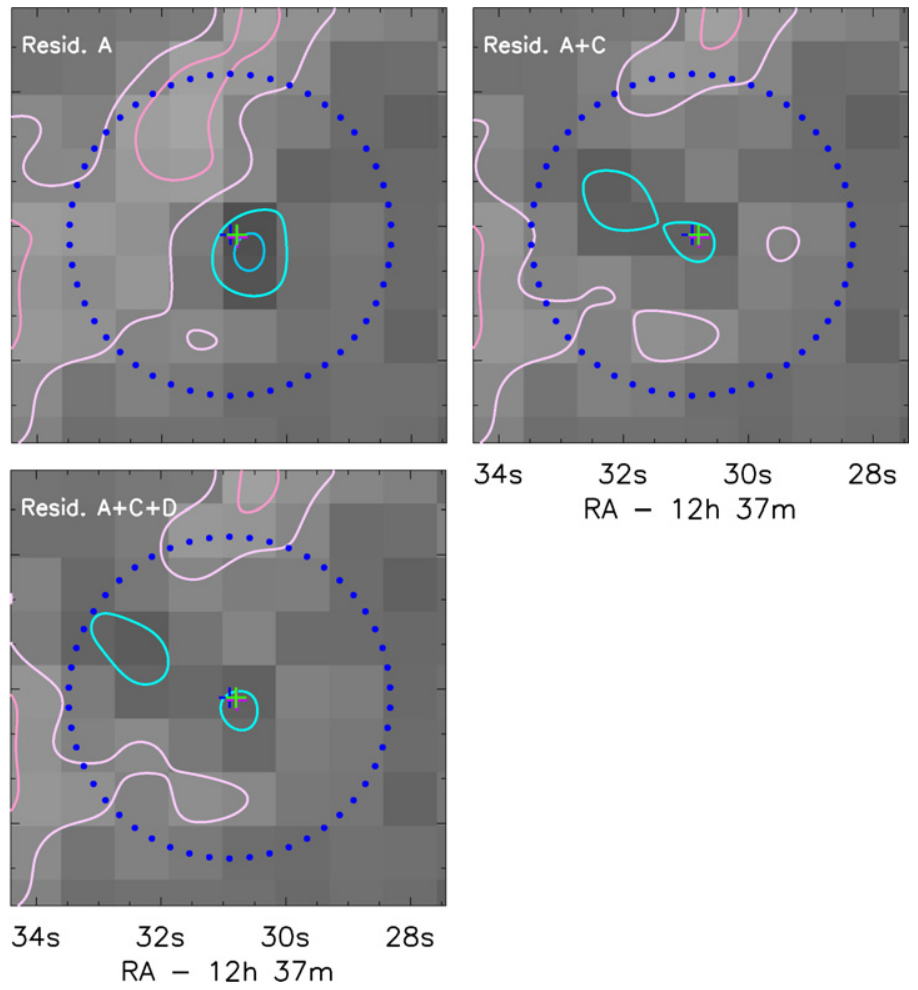

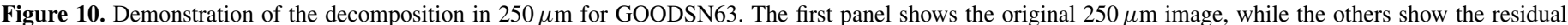

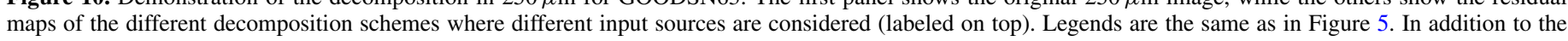

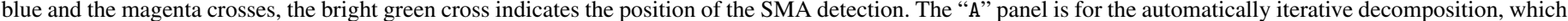

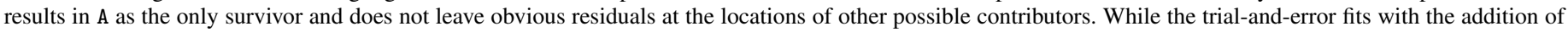

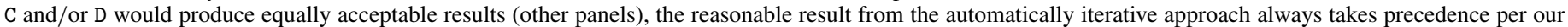
decomposition rule, and hence $\mathrm{A}$ is deemed to be the sole contributor to $250 \mu \mathrm{m}$ emission.

(A color version of this figure is available in the online journal.)

in the upper right panel of Figure 9). This database gives $z_{\mathrm{ph}}=2.4$ for this object, which agrees with $z_{\mathrm{ph}}=2.28$ quite well considering the different photometry in these two approaches. We adopt $z_{\mathrm{ph}}=2.28$ for the analysis below.

\subsubsection{Decomposition in Mid-to-far-IR}

The automatically iterative decomposition of GOODSN63 was successful in all bands from $250 \mu \mathrm{m}$ to $24 \mu \mathrm{m}$. In $24 \mu \mathrm{m}$, $\mathrm{B}$ has zero flux. We tried to decompose by subtracting A first, and found that the residual image had no flux left at the position of $\mathrm{B}$, consistent with the simultaneous fit. This probably is not surprising, because $\mathrm{B}$, while being brighter than A from $V_{606}$ to $H_{160}$, steadily becomes much fainter than A when moving to the redder and redder IRAC channels $(>1.5 \times$ fainter in $8.0 \mu \mathrm{m})$. The simultaneous fit in other bands also gives zero flux for $\mathrm{B}$. Similar to B, D also has zero flux in 100,160 , and $250 \mu \mathrm{m}$. Therefore, the two distinct sources in the 100 and $160 \mu \mathrm{m}$ described at the beginning of Section 4.2 should corresponds to $\mathrm{A}$ and $\mathrm{C}$, respectively.

The automatically iterative decomposition shows that $\mathrm{C}$ also has zero flux in $250 \mu \mathrm{m}$, leaving A as the sole object responsible for the emission in this band. The decomposition in this band is shown in Figure 10. The decomposition settled on A with a very reasonable error estimate and left no detectable fluxes at the positions of any other sources. We also tried the trial-and-error approach by adding C and/or D (although D probably should not be involved in the first place given its zero fluxes in 100 and $160 \mu \mathrm{m}$ ), which produced equally acceptable results. However, our decomposition rule is that the automatically iterative approach always takes precedence as long as the results are reasonable, and therefore we adopted $\mathrm{A}$ as the only contributor to the $250 \mu \mathrm{m}$ emission. This decision is also supported by the fact that the flux ratio of A to $\mathrm{C}$ gradually increases with the wavelengths, increasing from 0.77:1 at $24 \mu \mathrm{m}$ to $3.47: 1$ at $160 \mu \mathrm{m}$, consistent with the picture that $\mathrm{A}$ is much more dominant in the FIR. This also raises the point that one should not blindly take the brightest $24 \mu \mathrm{m}$ source within the Herschel beam as the counterpart.

The above results mean that it is possible to include the data in 350 and $500 \mu \mathrm{m}$ for further analysis, as there should not be any other neighbors that could contaminate the light from $A$ in these two bands. The final mid-to-far-IR flux densities of GOODSN63-A that we adopt are summarized in Table 2.

\subsubsection{Total IR Emission and Stellar Populations}

The A-only decomposition result above is supported by the SMA observation of this source (see Section 4.2; Barger et al. 2012), which reveals a single source whose position is on A-1. This is also supported by the VLA $1.4 \mathrm{GHz}$ data, where there is a source with $S_{1.4 \mathrm{GHz}}=0.123 \mathrm{mJy}$ coinciding with A. The radio position is at R.A. $=12^{\mathrm{h}} 37^{\mathrm{m}} 30^{\mathrm{s}} 78$, Decl. $=62^{\circ} 12^{\prime} 58^{\prime \prime} \cdot 7$, which is right in between $\mathrm{A}-1$ and $\mathrm{A}-2$ (Morrison et al. 2010). There is no X-ray detection of GOODSN63 in the 2Ms Chandra data (Alexander et al. 2003), which have the limit of $2.5 \times 10^{-17} \mathrm{erg} \mathrm{s}^{-1} \mathrm{~cm}^{-2}$ in the most sensitive $0.5-2 \mathrm{keV}$ band. At $z=2.28$, this corresponds to an upper limit of $1.0 \times 10^{42} \mathrm{erg} \mathrm{s}^{-1}$ in the rest-frame 1.6-6.6 keV, which is at 

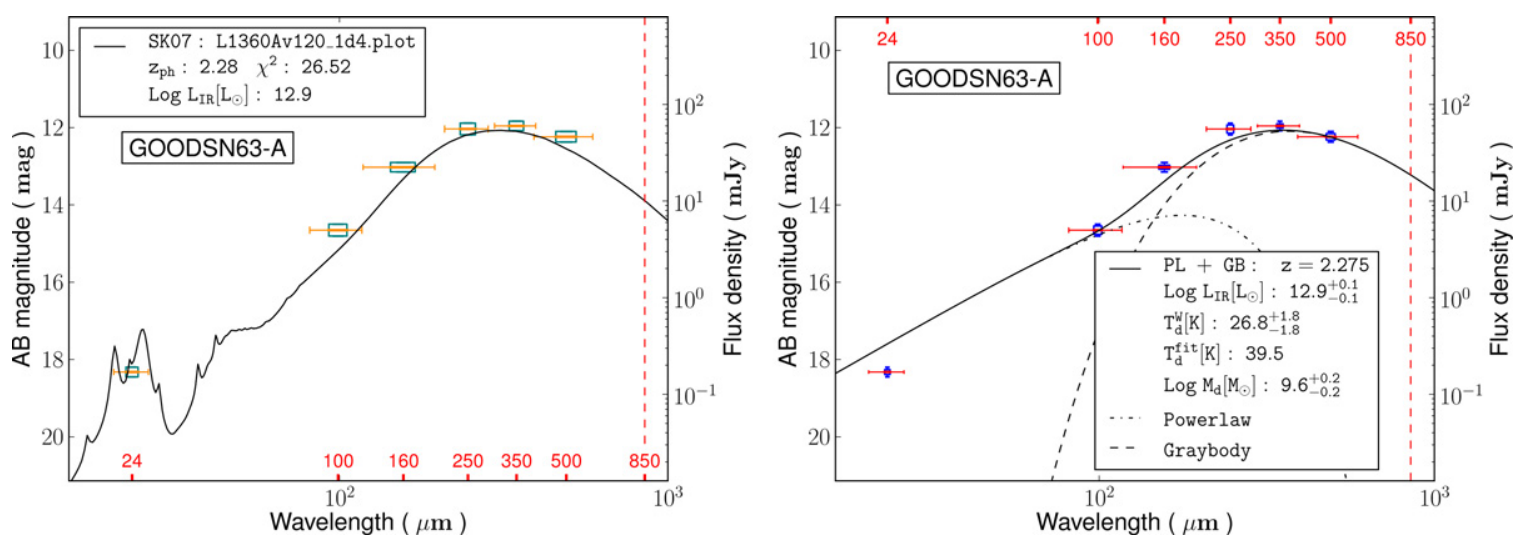

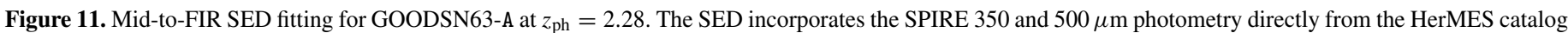

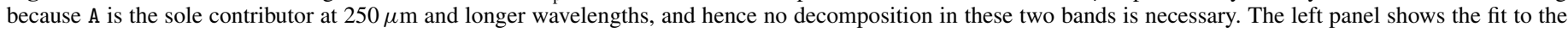
SK07 models, and the right panel shows the fit using the "power-law + graybody" models. Legends are the same as in Figure 6.

(A color version of this figure is available in the online journal.)

the borderline of AGNs. Therefore, we believe that the FIR emission of GOODSN63 is most likely powered by stars.

We derived $L_{\mathrm{IR}}$ using the mid-to-far-IR SED from 24 to $500 \mu \mathrm{m}$, fixing the redshift at $z_{\mathrm{ph}}=2.28$. The result is summarized in the left panel of Figure 11. The best-fit SK07 model is heavily dust-extincted, with $A_{V}=12.0 \mathrm{mag}$. We obtained $L_{\mathrm{IR}}=7.9 \times 10^{12} L_{\odot}$, and therefore this object is a ULIRG. The contribution from the dust re-processed light in the exposed region only amounts to $L_{\mathrm{IR}}^{\mathrm{ext}}=8.7 \times 10^{10} L_{\odot}$, and thus $L_{\mathrm{IR}}^{\mathrm{blk}}=7.8 \times 10^{12} L_{\odot}$ and $\mathrm{SFR}_{\mathrm{IR}}^{\mathrm{blk}}=781 M_{\odot} \mathrm{yr}^{-1}$. In contrast, as described in Section 4.2.2, it has $\mathrm{SFR}_{\mathrm{fit}}=11.2 M_{\odot} \mathrm{yr}^{-1}$, which is contributed solely by the blue component of A. The stellar mass of this system is dominated by the red subcomponent of A, which is $4.7 \times 10^{11} M_{\odot}$. Therefore, we get SSFR $=1.69 \mathrm{Gyr}^{-1}$ (or $T_{\mathrm{db}}^{\mathrm{tot}}=593 \mathrm{Myr}$ ), and $T_{\mathrm{db}}^{\mathrm{blk}}=602 \mathrm{Myr}$. The latter doubling time is vastly different from that inferred for GOODSN06, and is much longer than the typical lifetime of 10-100 Myr for a ULIRG. Nevertheless, it is still much shorter, $T=2.0 \mathrm{Gyr}$, than the exposed stellar population. The dust temperature and the dust mass of this system, $T_{d}=39.4 \mathrm{~K}$ and $M_{d}=3.6 \times 10^{9} M_{\odot}$, are typical of SMGs. The best-fit SK07 model predicts $S_{850}=$ $9.81 \mathrm{mJy}$, consistent with the actual observation. The dust mass implies a gas mass of $M_{\text {gas }}=5.6 \times 10^{11} M_{\odot}$, sufficient to fuel the ULIRG for the next $\sim 700 \mathrm{Myr}$.

The red subcomponent of A is a short burst ( $\tau=10 \mathrm{Myr}$ ) that is 2 Gyr old (Section 4.2.2), which implies that the majority of the existing stellar mass of this system was formed in an intense starbursting phase at $z>5$ and that the current ULIRG phase is an episode unrelated to the main build-up process of the existing system.

We also examined the FIR-radio relation for this system and obtained $q_{\mathrm{IR}}=2.29$. This is lower but still consistent with the mean value of $2.40 \pm 0.24$ of Ivison et al. (2010).

\section{SOURCES IN THE UDS}

The two sources in the UDS have much shallower SPIRE data than those in the GOODS-N because they are in a HerMES "L6" field. Unfortunately, the PACS data have not yet been released.

\subsection{UDS01 (UDS-J021806.0-051247)}

This source does not have data in the ACS, and we relied on the WFC3 IR data for the morphologies. The optical data from the CFHTLS-Wide were used in the optical-to-NIR SED analysis.

\subsubsection{Morphologies and Potential Components}

Figure 12 shows the images of UDS01. Within an $18^{\prime \prime}$ radius of the $250 \mu \mathrm{m}$ position, there are 35 objects in $H_{160}$ with $\mathrm{S} / \mathrm{N} \geqslant 5$. By comparing the $250 \mu \mathrm{m}$ and the $24 \mu \mathrm{m}$ images, we found that only nine of these objects could be significant contributors to the FIR flux. These objects are labeled from "A" to " $\mathrm{H}$ " according to their proximity to the $250 \mu \mathrm{m}$ centroid, which lies in between B and C. B has a spectroscopic redshift of $z=1.042$ (Bradshaw et al. 2013; McLure et al. 2013).

At $24 \mu \mathrm{m}$, these nine sources are already severely blended. Nevertheless, we can still roughly distinguish two major sets based on their positions. The morphological details of these two sets in $H_{160}$, hereafter the "southern" and the "northern" sets, are shown in Figure 13. The southern set includes A and B, which are 3.9 apart, and are $2 . .2$ and 2.5 from the $250 \mu \mathrm{m}$ centroid, respectively. A is a small, compact object. B is by far the more dominant of the two in both IRAC and MIPS bands. In $H_{160}$, it shows a dominant core and an extended, irregular halo that is suggestive of a post-merger. The "northern" set includes C, D, E, F, and G. The dominant member in this set, as seen from IRAC to MIPS, is D, while $\mathrm{C}$ is the next in line. These two objects are 2.8 and 3 .' 4 from the $250 \mu \mathrm{m}$ centroid, respectively, and they are 2 . 5 apart from each other. $\mathrm{C}$ has a spheroidal-like core, while $\mathrm{D}$ is more extended and has an irregular halo suggestive of a post-merger similar to B. E seems to be the companion of C, while G could be associated with D. F, on the other hand, already diminishes to invisible in $8 \mu \mathrm{m}$, and therefore is likely negligible at longer wavelengths. Finally, I and $\mathrm{H}$, which are small and compact and only 1".6 apart in $H_{160}$, form a distinct, but the least important, set.

\subsubsection{Optical-to-near-IR SED Analysis}

While the high-resolution WFC3 data have discerned the multiple objects in this region as discussed above, we cannot keep the same set of objects for the optical-to-NIR SED analysis due to the lack of the high-resolution ACS data in the optical. As a substitution, the CFHTLS-Wide data were used for the SED analysis. In principle, we could run TFIT on those data using the WFC3 data as the templates, however, this was not 

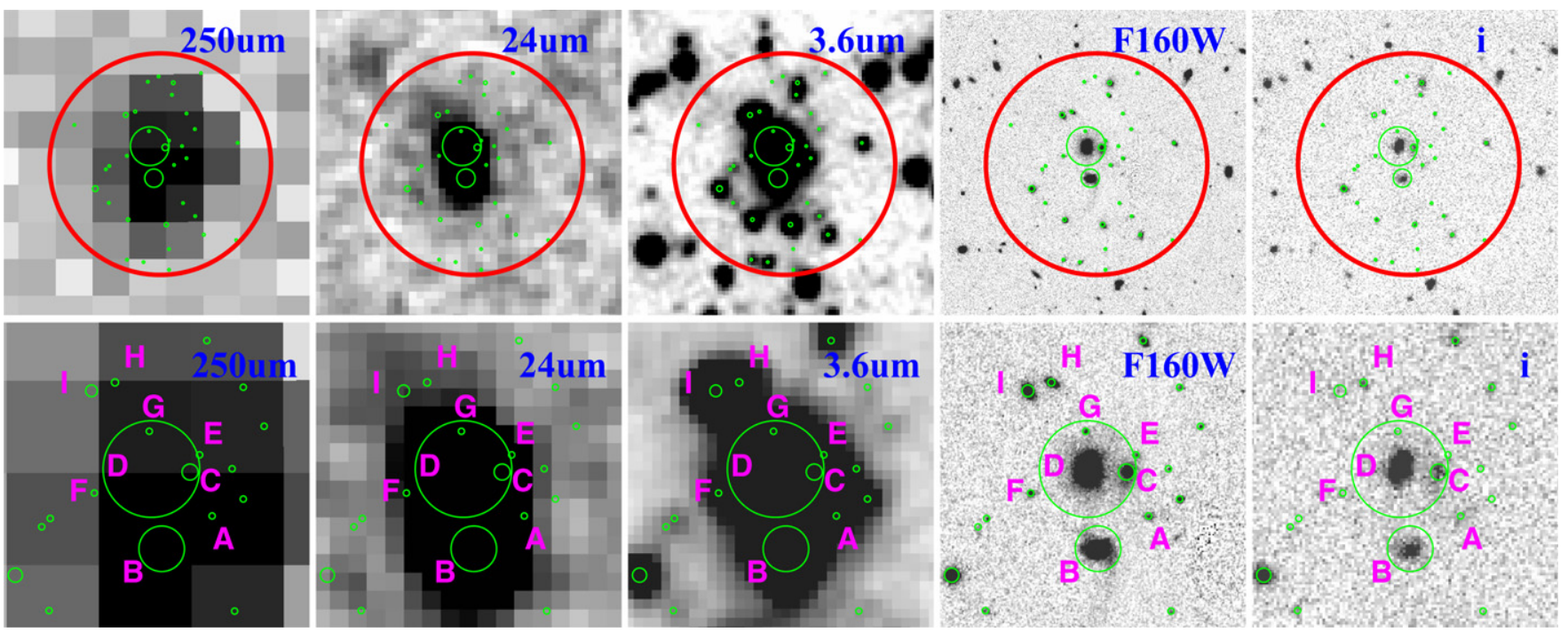

Figure 12. FIR to optical images of UDS01. The organization and the legends are the same as in Figure 2 . This source lacks 160,100 , and $70 \mu \mathrm{m}$ data. The $i$-band image is from the CFHTLS-Wide program.

(A color version of this figure is available in the online journal.)

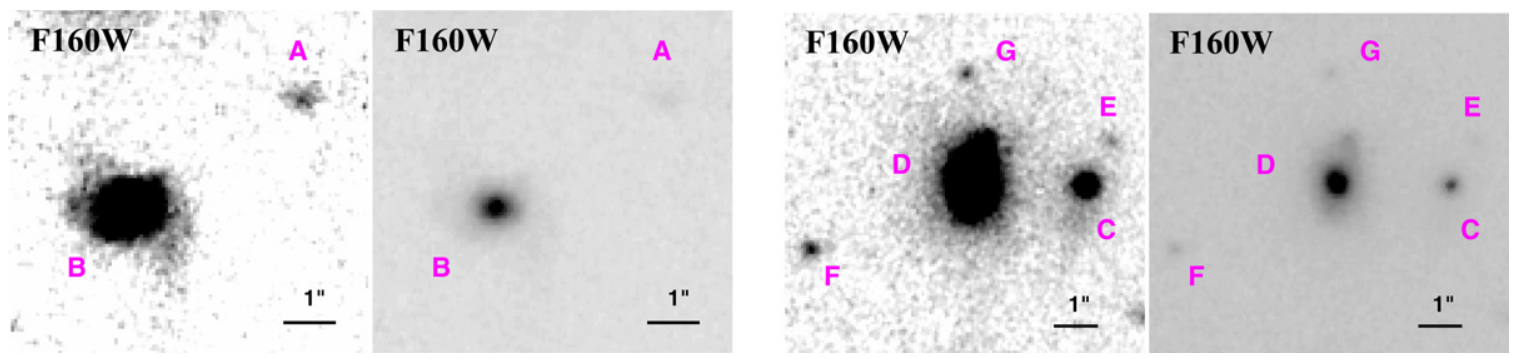

Figure 13. NIR morphologies of the possible contributors to UDS-01, shown in $H_{160}$ with two contrast levels to reveal different features. The left panel is for the "southern" group that includes A and B, while the right panel is for the "northern" group that includes objects from C to F.

(A color version of this figure is available in the online journal.)

practical because we discovered that the astrometric solution of the CFHTLS-Wide data is not entirely consistent with that of the CANDELS WFC3 data. As fixing this problem would be beyond the scope of this paper, our approach was to carry out independent photometry on the CFHTLS-Wide data alone and then to match these with the WFC3 detections for the SED construction. For this purpose, we used SExtractor in dual-image mode and the $i$-band image as the detection image. $\mathrm{C}$ and $\mathrm{E}$ cannot be separated in these images; thus, we take them as a single source and will refer to this source as $\mathrm{C} / \mathrm{E}$ hereafter. $\mathrm{E}$ is $>3$ mag fainter than $\mathrm{C}$ in $J_{125}$ and $H_{160}$, and hence is negligible for the analysis of the stellar population. D and $G$ are taken as a single source as well for the same reason; $G$ is $>5$ mag fainter than D in the WFC3, and hence likely negligible. We refer to them as D/G hereafter. I is not visible in the CFHTLS data and thus has to be excluded from this analysis. The colors of these objects were measured in the MAG_ISO apertures, and the $i$-band MAG_AUTO magnitudes were used as the reference to convert the colors to the magnitudes for the SED construction. In the nearIR, we used the CANDELS WFC $3 J_{125}$ and $H_{160}$, and these magnitudes are obtained in the same way as in Section 4. In the longer wavelengths, we used the IRAC 3.6 and $4.5 \mu \mathrm{m}$ data from the SEDS program. The objects are severely blended in the IRAC images, and hence we had to deblend them by using TFIT to obtain reliable photometry of the individual objects. As in Section 4, the $H_{160}$ image was used as the template for TFIT.
The photometric redshift results are summarized in Figure 14. A is excluded because this faint object is not well detected in the CFHTLS-Wide data. Formally, it has $z_{\mathrm{ph}}=3.5$; however, this is not trustworthy because of the large photometric errors. As shown in the decomposition below, this object is irrelevant. $\mathrm{B}$ has $z_{\mathrm{ph}}=1.05$, which agrees very well with its $z_{\mathrm{spec}}=1.042 . \mathrm{C} / \mathrm{E}$ and D/G have $z_{\mathrm{ph}}=0.98$ and 1.09 , respectively, and in terms of $\Delta z /(1+z)$ they agree to $z_{\text {spec }}$ of B to 0.02 and 0.03 , respectively, well within the accuracy of $z_{\mathrm{ph}}$ that can be achieved. The peaks of their $P(z)$ distributions overlap significantly. All this means that these three objects are very likely at the same redshifts and associated. C/E and D/G are separated by 2".52, and B is 5.25 away from them. At $z=1.042$, these corresponds to 20.5 and $42.7 \mathrm{kpc}$, respectively, and are within the scale of galaxy groups.

The object of the highest stellar mass is $D / G$, which has $8.3 \times 10^{10} M_{\odot}$. It has a prolonged SFH with $\tau=0.5 \mathrm{Gyr}$, and its best-fit age is $T=1.0 \mathrm{Gyr}$. It has $\mathrm{SFR}_{\mathrm{fit}}=39.0 M_{\odot} \mathrm{yr}^{-1}$. All this suggests that the stellar mass assembly in $D / G$ is a slow, gradual process. In contrast, B is best explained by a young SSP with an age of $130 \mathrm{Myr}$, and the inferred stellar mass is $4.6 \times 10^{10} M_{\odot}$. This indicates that it built up its stellar mass through a sudden onset of intense star formation. Similarly, C/E is best fit by a young stellar population with an age of $180 \mathrm{Myr}$ and a short episode of SFH that has $\tau=50$ Myr. However, its stellar mass is almost one magnitude lower at $5.4 \times 10^{9} M_{\odot}$. 


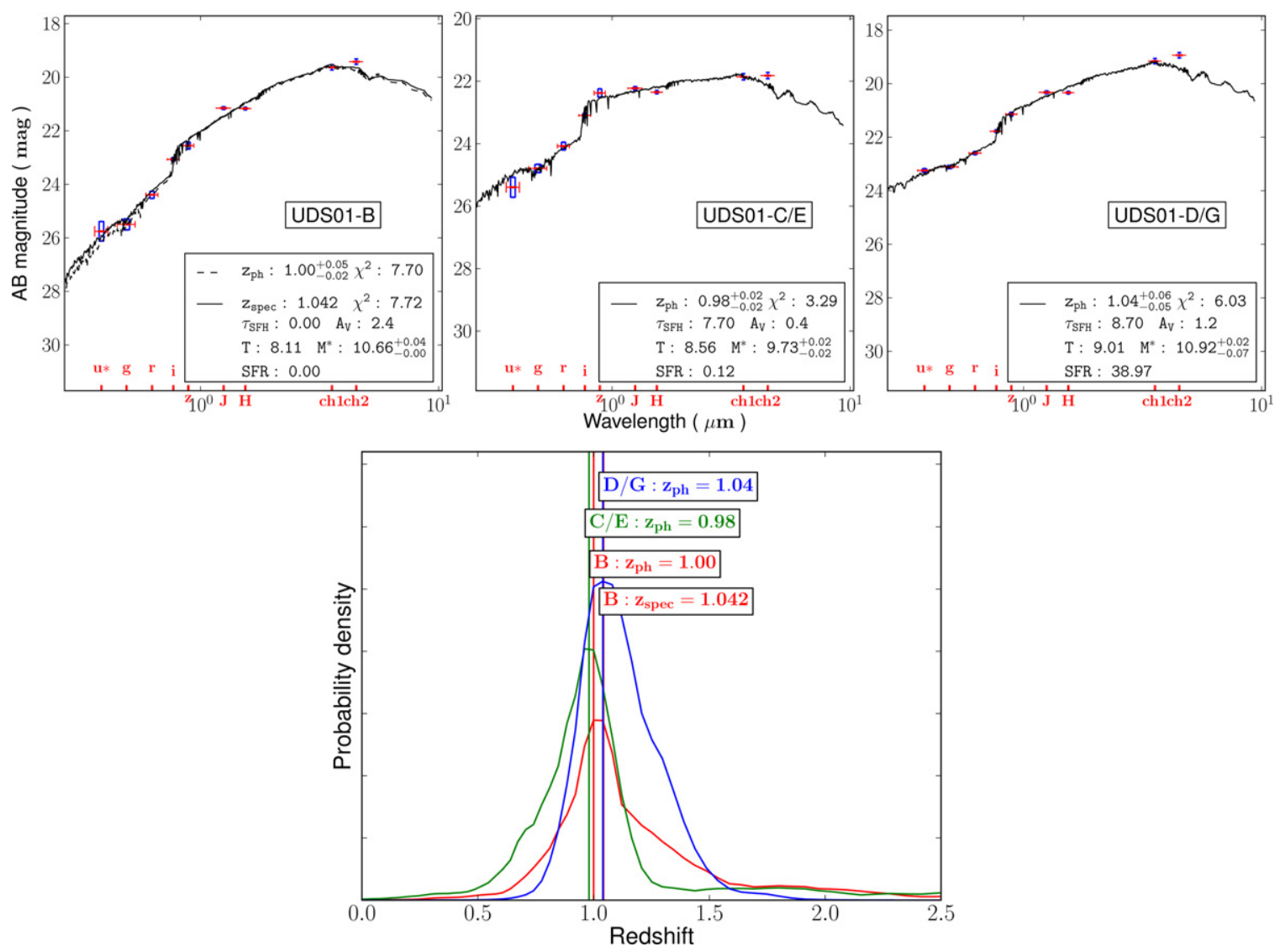

Figure 14. Optical-to-NIR SED analysis of the possible contributors to UDS01. Legends are the same as in Figure 4. A is excluded here because it is not well detected in CFHTLS-Wide data. It is highly plausible $\mathrm{C} / \mathrm{E}$ and $\mathrm{D} / \mathrm{G}$ are at the same redshift as $\mathrm{B}$, which has $z_{\mathrm{spec}}=1.042$.

(A color version of this figure is available in the online journal.)

\subsubsection{Decomposition in Mid-to-far-IR}

We used the position priors in $H_{160}$ for the decomposition. Not surprisingly, the automatically iterative fit in $24 \mu \mathrm{m}$ converged on only $\mathrm{B}$ and $\mathrm{D}$, the latter of which is by far the most dominant object in $24 \mu \mathrm{m}$. In $250 \mu \mathrm{m}$, however, it settled on B and C with the resulted flux ratio of $55.5 \%: 44.5 \%$, and rejected D because of its negligible flux.

Although in the case of GOODSN63 we have learned that the strongest $24 \mu \mathrm{m}$ source is not necessarily the major contributor to the FIR emission, it seems unusual that a FIR source would have no $24 \mu \mathrm{m}$ counterpart. We thus checked for degeneracy using the trail-and-error method. This confirmed that indeed only B, C, and D could be relevant. The results are summarized in Figure 15. The forced fit with $B$ and $D$ left slightly larger residual than the automatically iterative result (" $\mathrm{B}+\mathrm{C}$ "), however, it is not unacceptable. Fitting with only $C$ or D, on the other hand, left obvious residual at the location of B, clearly indicating that a reasonable solution must include B. Forcing the fit to include B, $\mathrm{C}$ and $\mathrm{D}$ produced the same result as the $\mathrm{B}+\mathrm{C}$ case. Therefore, our decomposition slightly favors $\mathrm{B}$ and $\mathrm{C}$ as the contributors to the FIR emission, however, we cannot definitely rule out the possibility that it actually is B and D that are responsible.

One would hope that the radio data from the $100 \mathrm{mJy}$ VLA 1.5 GHz survey (Simpson et al. 2006) could break this degeneracy. Their catalog contains only one strong radio source in the area occupied by UDS01, with $S_{1.5 \mathrm{GHz}}=0.185 \pm$ $0.030 \mathrm{mJy}$. The radio position is R.A. $=2^{\mathrm{h}} 18^{\mathrm{m}} 06^{\mathrm{s}} .16$, Decl. $=$ $-5^{\circ} 12^{\prime} 45^{\prime \prime} 61$ (J2000), which is right on D (only offset by $0^{\prime \prime} 68$ ). While this VLA survey used the B and C configurations and thus has worse angular resolution than that of Morrison et al.'s data in the GOODS-N, its positional accuracy is still good to the subarcsecond level for the high $\mathrm{S} / \mathrm{N}$ radio sources such as this one. For this reason, D seems to be a more plausible FIR contributor than C. However, it is puzzling that B, which is the strongest in $250 \mu \mathrm{m}$ among all, does not have a radio counterpart in the catalog.

The flux densities of $\mathrm{B}$ and $\mathrm{D}$, based on the decomposition results using the $B+D$ case, are summarized in Table 2. The flux densities of $B$ in the $B+C$ case only differ by $<6 \%$.

\subsubsection{Total IR Emission and Stellar Populations}

None of the above components are in the SXDS X-ray catalog of Ueda et al. (2008), which has a sensitivity limit of $6 \times 10^{-16} \mathrm{erg} \mathrm{s}^{-1} \mathrm{~cm}^{-2}$ in its most sensitive $0.5-2 \mathrm{keV}$ band. At $z \sim 1$, this corresponds to an upper limit of $\sim 3.2 \times 10^{42} \mathrm{erg} \mathrm{s}^{-1}$ in rest-frame $1-4 \mathrm{keV}$. While this cannot rule out the possibility of AGN contamination, there is no strong evidence suggesting that there could be an AGN. Therefore, we take it that the FIR emission of UDS01 is all due to star formation.

Regardless of the exact counterparts, it is clear that the FIR emission is due to the interacting system that includes B, C, and D. To estimate $L_{\mathrm{IR}}$, we adopted $z_{\text {spec }}=1.042$ of B as their common redshift, and fit the decomposed $250 \mu \mathrm{m}$ and $24 \mu \mathrm{m}$ fluxes to the SK07 models. As we only had two data points, we did not apply scaling to the templates but simply read off the luminosities of the best-fit templates (Figure 16). In the case of $\mathrm{B}+\mathrm{C}$, we obtained $L_{\mathrm{IR}}=1 \times 10^{12} L_{\odot}$ for B, and did not estimate for $\mathrm{C}$ because it only has one data point $(250 \mu \mathrm{m})$. In the case 

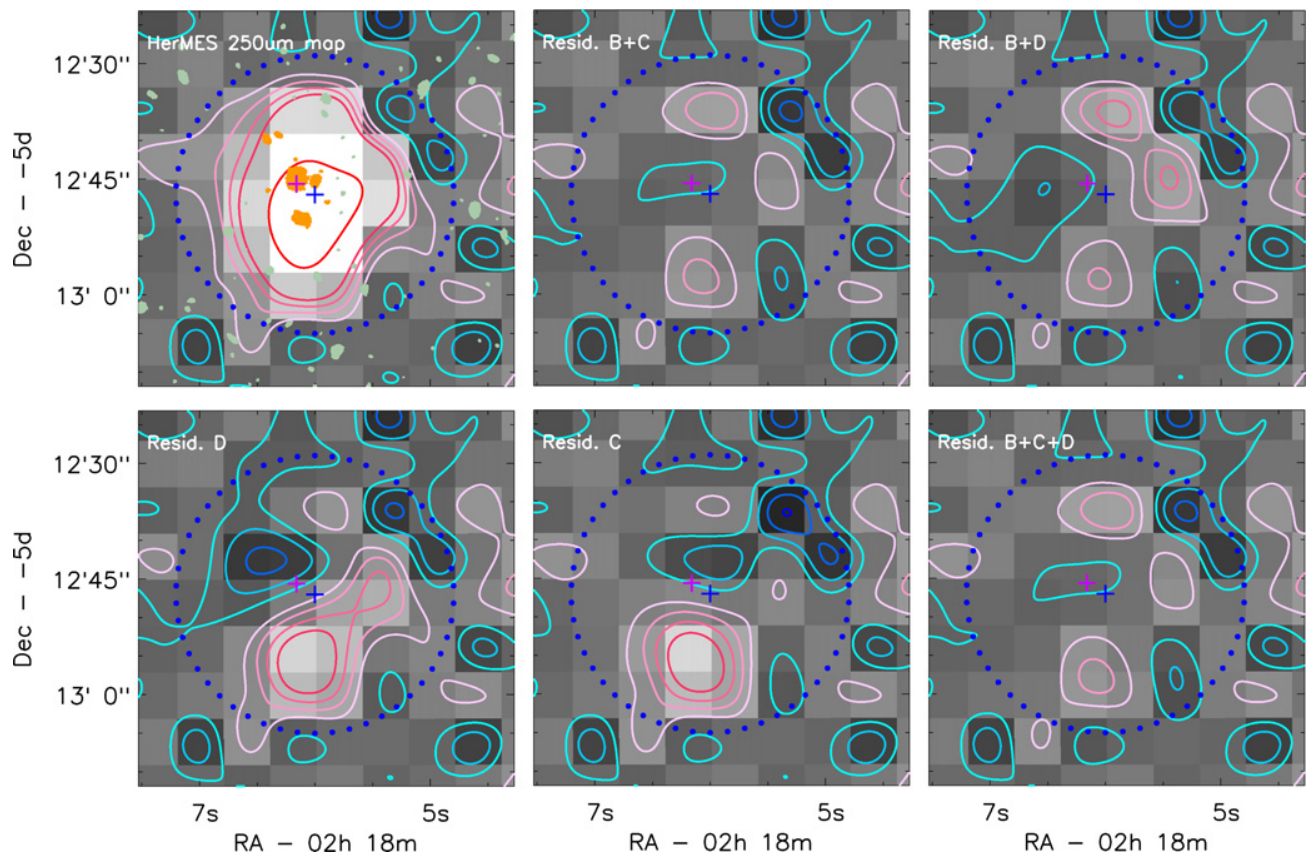

Figure 15. Demonstration of the decomposition in $250 \mu \mathrm{m}$ for UDS01. The first panel shows the original $250 \mu \mathrm{m}$ image, while the others show the residual maps of the different decomposition schemes where different input sources are considered (labeled on top). Legends are the same as in Figure 5. This is a degenerate case. While automatically iterative fit settles on $\mathrm{B}+\mathrm{C}$, the trial-and-error run using $\mathrm{B}+\mathrm{D}$ still produces satisfactory results. However, the fit without the inclusion of $\mathrm{B}$ are not acceptable.

(A color version of this figure is available in the online journal.)
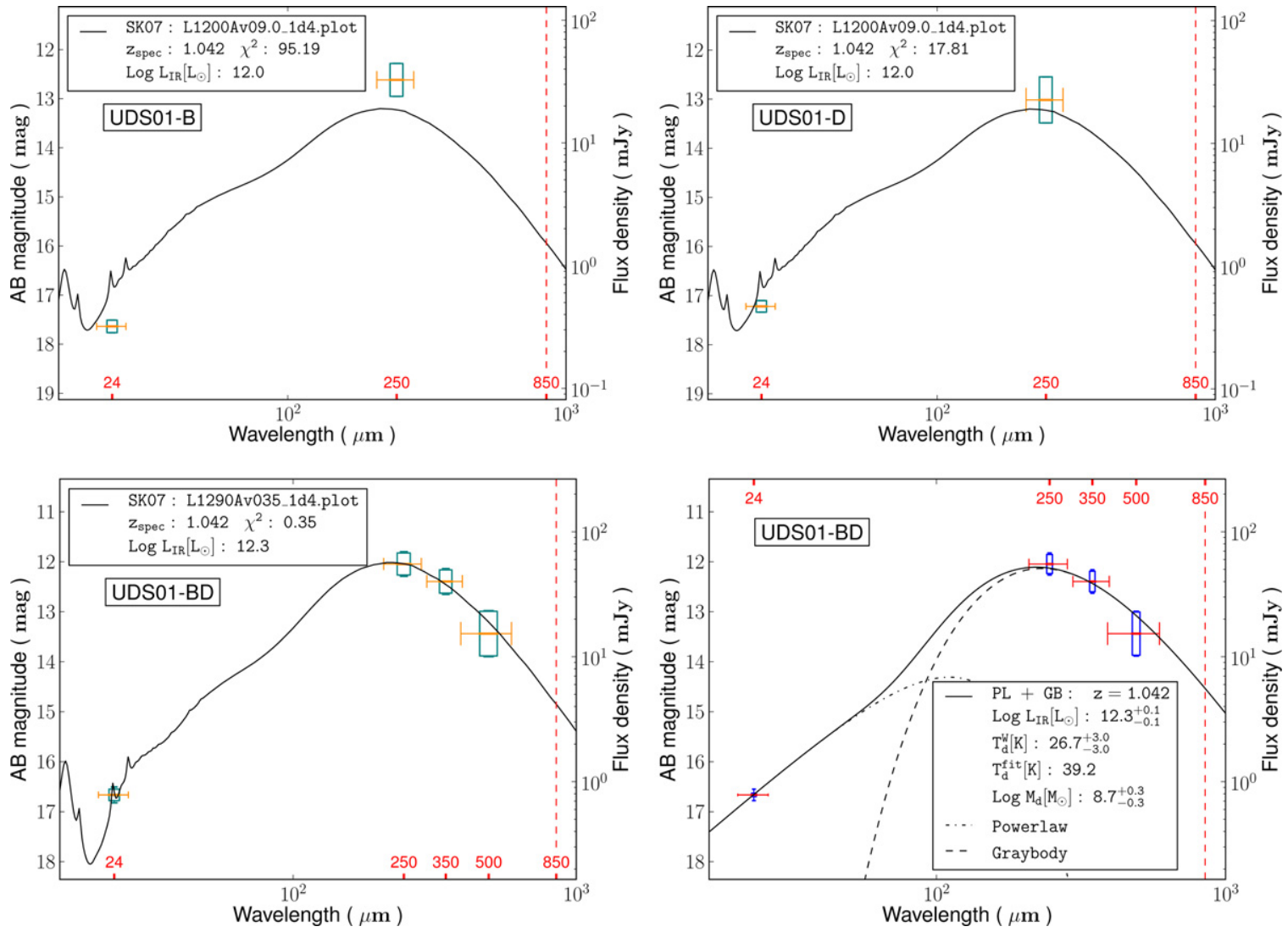

Figure 16. Mid-to-FIR SED fitting for UDS01 at $z=1.042$. Legends are the same as in Figure 6. The two figures on the top show the fits to the SK07 models in the case where B and D are the counterparts. No scaling is applied because there are only two data points available (see the text). In the degenerate case where B and C are the counterparts, the result for B is exactly the same as shown here, however, it is impossible to solve for $\mathrm{C}$ as it has only one data point available (lacking $24 \mu \mathrm{m}$ ). The two figures on the bottom row show the fitting results when this system is treated as a whole such that the 350 and $500 \mu \mathrm{m}$ data can also be used without doing decomposition: the one to the left is the fit to the SK07 models, while the one to the right is the fit to the power-law + graybody models.

(A color version of this figure is available in the online journal.) 


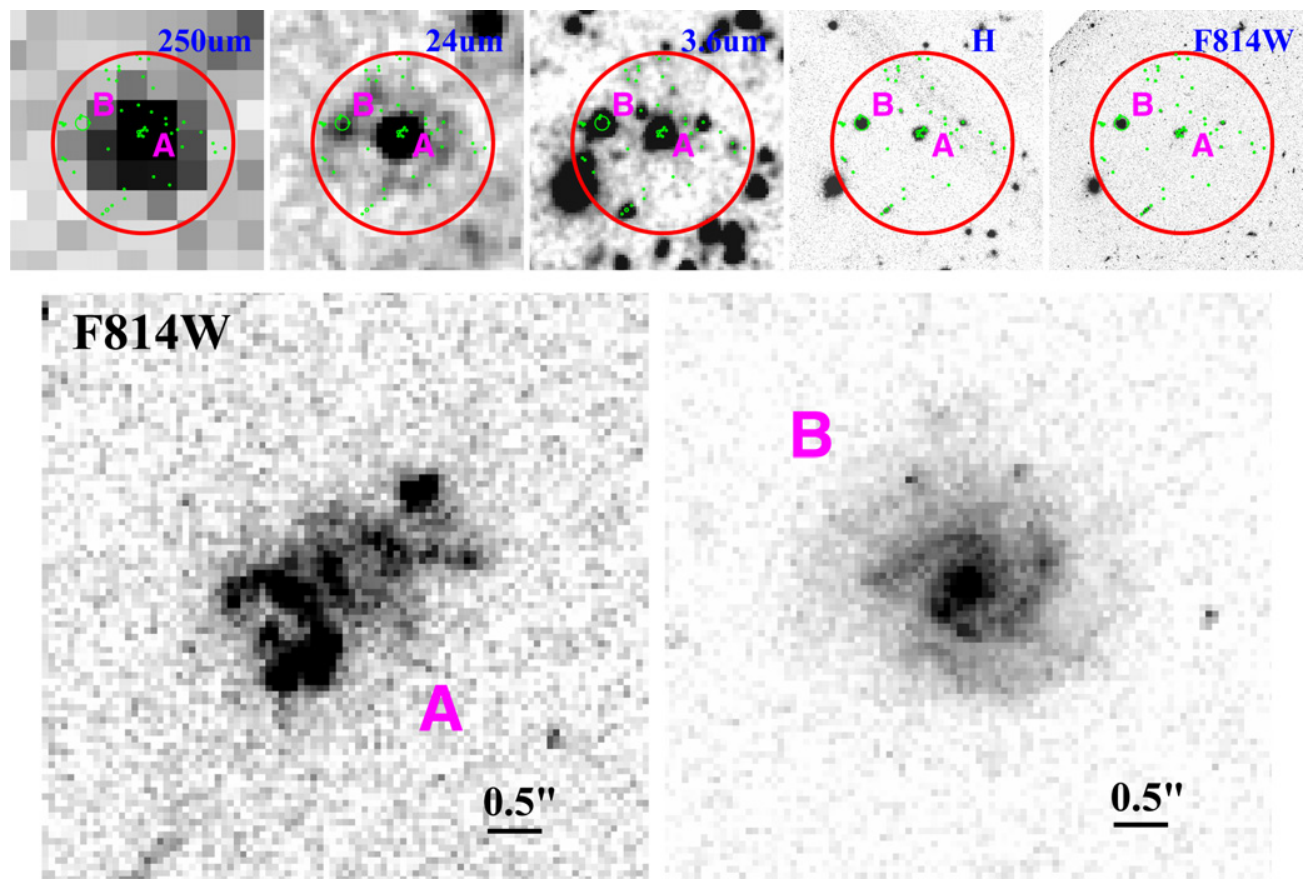

Figure 17. FIR to optical images of UDS04 are shown in the top row. The legends are the same as in Figure 2. As there are only two potential contributors (A and B) to the FIR emission, no zoomed-in view of their vicinity is shown. This source lacks 160,100 , and $70 \mu \mathrm{m}$ data. The $H$-band image is from UKIDSS. The morphological details of A and B as revealed by the ACS $I_{814}$ image are shown in the bottom panel.

(A color version of this figure is available in the online journal.)

of $\mathrm{B}+\mathrm{D}$, the best-fit template is the same for both objects, and is the same as in the previous case. This is shown in the upper panel in Figure 16. It thus seems robust that $B$ is a ULIRG with $L_{\mathrm{IR}}=1 \times 10^{12} L_{\odot}$. We calculated $L_{\mathrm{IR}}^{\mathrm{ext}}=5.2 \times 10^{11} L_{\odot}$ and $L_{\mathrm{IR}}^{\mathrm{blk}}=4.8 \times 10^{11} L_{\odot}$, which implies $\mathrm{SFR}_{\mathrm{IR}}^{\mathrm{blk}}=48 M_{\odot} \mathrm{yr}^{-1}$. The SED fitting in Section 5.1.2 shows that its exposed stellar population is an SSP and thus $\mathrm{SFR}_{\mathrm{fit}}=0$. Therefore, we get $\mathrm{SFR}_{\mathrm{tot}}=48 M_{\odot} \mathrm{yr}^{-1}$. In addition, D could also be a ULIRG of the same $L_{\mathrm{IR}}=1 \times 10^{12} L_{\odot}$. It has $L_{\mathrm{IR}}^{\mathrm{ext}}=3.8 \times 10^{11} L_{\odot}$ and $L_{\mathrm{IR}}^{\mathrm{blk}}=6.2 \times 10^{11} L_{\odot}$, which implies $\mathrm{SFR}_{\mathrm{IR}}^{\mathrm{blk}}=62 \mathrm{M}_{\odot} \mathrm{yr}^{-1}$. Its exposed region has $\mathrm{SFR}_{\mathrm{fit}}=39 M_{\odot} \mathrm{yr}^{-1}$, and thus the whole system of D has $\mathrm{SFR}_{\mathrm{tot}}=101 M_{\odot} \mathrm{yr}^{-1}$.

As all the possible counterparts could be at the same redshift, we also considered the case where they were combined. In this case, we used the reported 350 and $500 \mu \mathrm{m}$ flux densities for UDS01 as a whole without doing decomposition. The fit, as shown in the lower panel of Figure 16, gives $L_{\mathrm{IR}}=$ $2.0 \times 10^{12} L_{\odot}$, which is exactly the value if we were to combine the luminosities obtained in the case of $B+D$ for the two objects individually.

The stellar mass of B obtained in Section 5.1.2 is $4.6 \times$ $10^{10} M_{\odot}$, and therefore it has SSFR $=1.0 \mathrm{Gyr}^{-1}, T_{\mathrm{db}}^{\text {tot }}=T_{\mathrm{db}}^{\mathrm{blk}}=$ $1.0 \mathrm{Gyr}$. This is a factor of 7.7 longer than the age (129 Myr) of the stellar population in the exposed region and is also much longer than the typical lifetime of a ULIRG. The stellar mass of $\mathrm{D}$ is $8.3 \times 10^{10} M_{\odot}$, and therefore in the $\mathrm{B}+\mathrm{D}$ case it has $\mathrm{SSFR}_{\mathrm{IR}}=1.2 \mathrm{Gyr}^{-1}, T_{\mathrm{db}}^{\text {tot }}=822 \mathrm{Myr}$ and $T_{\mathrm{db}}^{\mathrm{blk}}=1.3 \mathrm{Gyr}$. The latter is comparable to $T=1.0 \mathrm{Gyr}$ of the stellar population in the exposed region, however, is much longer than the typical lifetime of a ULIRG. We also derived $T_{d}^{\text {fit }}=39.2 \mathrm{~K}$ and $M_{d}=5.0 \times 10^{8} M_{\odot}$ for the whole, combined system. The latter implies a total gas mass of $M_{\text {gas }}=7.0 \times 10^{10} M_{\odot}$, which would be sufficient to fuel B to double its mass but would fall short for D. Generally speaking, the ULIRG phase of this whole system will only play a minor role in assembling its stellar mass.

We can check on the FIR-radio relation for $\mathrm{D}$, assuming that the real solution is the $\mathrm{B}+\mathrm{D}$ case. We obtained $q_{\mathrm{IR}}=2.00$, which is significantly lower than the mean of $2.40 \pm 0.24$. There is no radio source in the catalog of Simpson et al. (2006) that corresponds to B. We can place an upper limit of $0.1 \mathrm{mJy}$ at $1.5 \mathrm{GHz}$, which is the flux density limit of this catalog. This would then result in $q_{\mathrm{IR}}<1.91$ for B.

\subsection{UDS04 (UDS-J021731.1-050711)}

This source does not have WFC3 IR data because it is outside of the CANDELS WFC3 footprint. Therefore, we used UKIRT $J H K s$ data for the SED analysis in the near-IR. Fortunately, it has CANDELS ACS data for morphological information.

\subsubsection{Morphologies and Potential Components}

Figure 17 shows the image of UDS04, the UKIDSS $H$, and the ACS $I_{814}$. We searched for its potential components in the $I_{814}$ image, and found 50 objects within $18^{\prime \prime}$ to the $250 \mu \mathrm{m}$ position that have $\mathrm{S} / \mathrm{N}>5$ in $I_{814}$ and $\mathrm{S} / \mathrm{N}>3$ in $V_{606}$. The extra detection criterion in $V_{606}$ is to reject any cosmic-ray and image defect residuals.

From Figure 17, it is obvious that UDS04 corresponds to only two possible sources in $24 \mu \mathrm{m}$. The more dominant one, which coincides better with the UDS04 centroid, is actually made of eight tightly packed objects detected in $I_{814}$. The detail of this region is shown in the bottom left panel of Figure 17, and it is clear that these eight objects are actually the result of a very disturbed system being resolved. From its morphology alone, it is consistent with being a disk system undergoing a violent disk instability (e.g., Dekel et al. 2009). We designate this system as object A. As it turns out, it has spectroscopic 


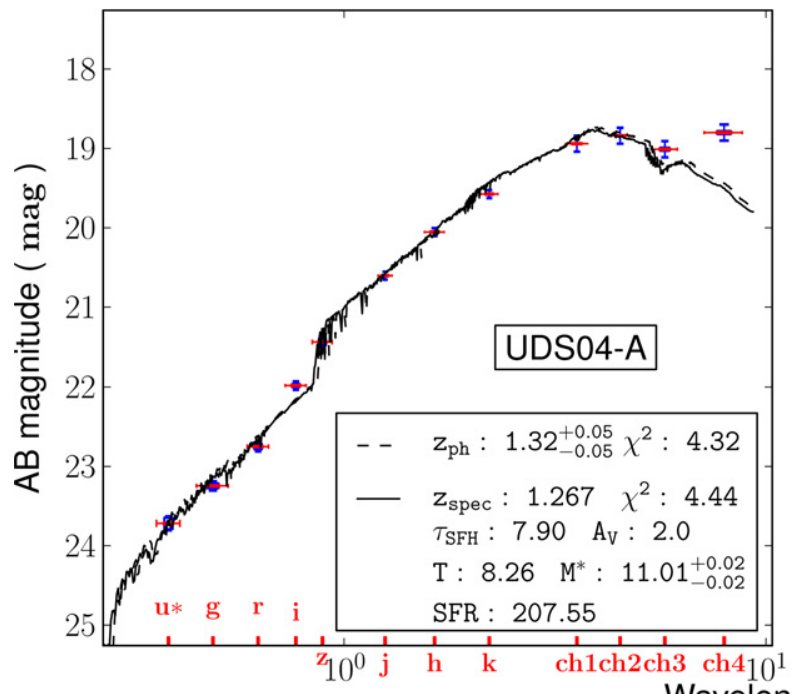

$$
\text { Wavelength }(\mu \mathrm{m})
$$

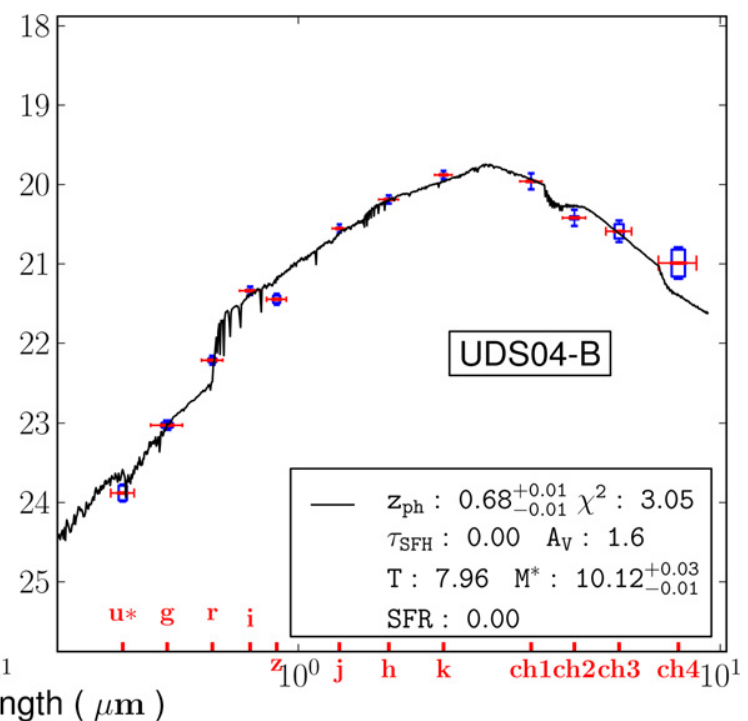

Figure 18. Optical-to-NIR SED analysis of the possible contributors to UDS04. Legends are the same as in Figure 4. B is unlikely to be at the same redshift as A. (A color version of this figure is available in the online journal.)

redshift of $z=1.267$ (Simpson et al. 2006; M. Akiyama et al., in preparation). The other $24 \mu \mathrm{m}$ source is much further away from the $250 \mu \mathrm{m}$ centroid, and is made of three $I_{814}$ objects. The $I_{814}$-band image shows that they are actually a normal spiral galaxy and two bright knots on its arms. We designate this spiral as B. This source does not have spectroscopic redshift.

\subsubsection{Optical-to-near-IR SED Analysis}

For the optical part of the SED, we only used the CFHTLSWide $u^{*}$ griz data as in Section 5.1 but did not include the ACS $V_{606}$ and $I_{814}$ data because the ACS images break A and B into subcomponents and are not straightforward for obtaining the total light. On the other hand, A and B are detected as single objects in the CFHTLS-Wide images, which is more appropriate in this context. As both objects lack WFC3 IR data, the UKIDSS data were used for the near-IR part of the SED. We carried out the photometry on the UKIDSS DR8 images, using the $K_{s}$ band as the detection band. Following the case in the optical, we used MAG_ISO for colors and then used the $K_{s}$-band MAG_AUTO magnitude for normalization. In the longer wavelengths, we used the same IRAC data as in Section 5.1.

The SED fitting results are summarized in Figure 18. For A, we derived $z_{\mathrm{ph}}=1.32$, which is in good agreement with its $z_{\text {spec }}=1.267(\Delta z /(1+z)=0.02)$. Fixing the redshift at its $z_{\text {spec }}$, the stellar mass thus derived is $1.0 \times 10^{11} M_{\odot}$. The best-fit stellar population has $\tau=79.4 \mathrm{Myr}$ and the age of $T=182 \mathrm{Myr}$. It also gives $\mathrm{SFR}_{\mathrm{fit}}=208 M_{\odot} \mathrm{yr}^{-1}$. Therefore, the whole life of this young galaxy up to this point has been in an intense starbursting phase.

For B we get $z_{\mathrm{ph}}=0.68$. Thus B cannot be associated with A.

\subsubsection{Decomposition in Mid-to-far-IR}

While aperture photometry would be sufficient in $24 \mu \mathrm{m}$, we still performed the same PSF fitting technique to derive the fluxes for A and B. As both objects are resolved into multiple components in the ACS data, we used their centroids as measured in the CFHTLS-Wide data to avoid any ambiguity. The automatically iterative decomposition in $250 \mu \mathrm{m}$ shows that the contribution from B is negligible and that A should be the only source responsible for the FIR emission. Thus we adopted the results from the HerMES DR1 catalog for the three SPIRE bands. Table 2 lists these results.

\subsubsection{Total IR Emission and Stellar Populations}

UDS04 is not detected in the SXDS X-ray data (Ueda et al. 2008). At $z=1.267$, the sensitivity limit of this catalog corresponds to an upper limit of $5.8 \times 10^{42} \mathrm{erg} \mathrm{s}^{-1}$ in rest-frame 1.1-4.5 keV. Based on the same argument as in Section 5.1.4, we take it that the FIR emission of UDS04, which is all from A, is all due to star formation. The mid-to-far-IR SED fitting results are summarized in Figure 19. Similar to the case in GOODSN63 where there is only one contributor to the FIR emission, we incorporated the SPIRE 350 and $500 \mu \mathrm{m}$ photometry taken directly from the HerMES DR 1 catalog. Using the SK07 templates, we obtained $L_{\mathrm{IR}}=5.0 \times 10^{12} L_{\odot}$ and thus it is an ULIRG. From the result in Section 5.2.2, we also obtained $L_{\mathrm{IR}}^{\mathrm{ext}}=2.55 \times 10^{12} L_{\odot}$. Therefore, $L_{\mathrm{IR}}^{\mathrm{blk}}=2.55 \times 10^{12} L_{\odot}$ and $\mathrm{SFR}_{\mathrm{IR}}^{\text {blk }}=255 M_{\odot} \mathrm{yr}^{-1}$. It has $\mathrm{SFR}_{\mathrm{fit}}=208 M_{\odot} \mathrm{yr}^{-1}$, and thus $\mathrm{SFR}_{\mathrm{tot}}=463 M_{\odot} \mathrm{yr}^{-1}$. As derived in Section 5.2.2, the exposed region has the stellar mass of $1.0 \times 10^{11} M_{\odot}$, which leads to SSFR $=4.6 \mathrm{Gyr}^{-1}, T_{\mathrm{db}}^{\mathrm{tot}}=216 \mathrm{Myr}$, and $T_{\mathrm{db}}^{\mathrm{blk}}=392 \mathrm{Myr}$. The exposed region has the best-fit age $T=182 \mathrm{Myr}$ and the declining timescale $\tau=79$ Myr. In this sense, UDS04-A is similar to GOODSN06-A: this young system has been in a starbursting phase ever since its birth, and while the star formation in the exposed region is quickly winding down, it is still sustained in the dust-blocked region. As shown in the right panel of Figure 19, we derived $T_{d}^{\text {fit }}=38.2 \mathrm{~K}$ and $M_{d}=1.0 \times 10^{9} M_{\odot}$. The latter implies $M_{\mathrm{gas}}=1.4 \times 10^{11} M_{\odot}$, which would be sufficient to fuel the dust-blocked region for the next $550 \mathrm{Myr}$ to more than double the stellar mass.

A is detected in the $100 \mu \mathrm{Jy} 1.5 \mathrm{GHz}$ data (Simpson et al. 2006), whose radio location is at R.A. $=2^{\mathrm{h}} 17^{\mathrm{m}} 31^{\mathrm{s}} \cdot 17$, Decl. $=$ $-5^{\circ} 07^{\prime} 09^{\prime \prime} 35$ (J2000) and is only 0'.26 away from the centroid of A. It has $S_{1.5 \mathrm{GHz}}=1.055 \pm 0.013 \mathrm{mJy}$ and is the strongest radio source in our sample. We derived $q_{\mathrm{IR}}=1.74$, which is much lower than $2.40 \pm 0.24$. 

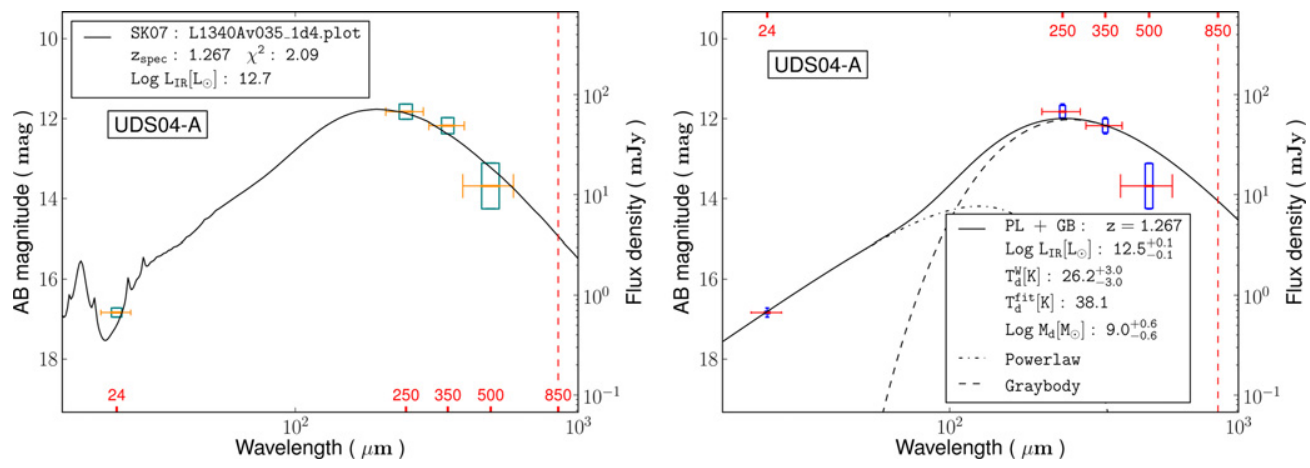

Figure 19. Mid-to-FIR SED fitting for UDS04-A at $z_{\text {spec }}=1.267$. The SED incorporates the SPIRE 350 and $500 \mu$ m photometry directly from the HerMES catalog because $\mathrm{A}$ is the sole contributor at $250 \mu \mathrm{m}$ and longer wavelengths, and hence no decomposition in these two bands are necessary. The left panel shows the fit to the SK07 models, and the right panel shows the fit using the power-law + graybody models. Legends are the same as in Figure 6.

(A color version of this figure is available in the online journal.)

\section{SOURCES IN THE EGS}

As mentioned in Section 2, the EGS field has both the HerMES SPIRE and PEP PACS data. For the latter, we use the PEP DR1 "blind" (i.e., constructed without using the positions priors from the MIPS $24 \mu \mathrm{m}$ data) $100 \mu \mathrm{m}$ and $160 \mu \mathrm{m}$ catalogs. When this work started, the CANDELS WFC3 observations in this field were not yet finished and none of the sources in this field had WFC3 data. Therefore, we relied on the ACS $V_{606}$ and $I_{814}$ images from the AEGIS program to study their morphologies. To define the possible components, on the other hand, we used the CFHTLS-Deep $u^{*}$ griz images. We made this choice because the CFHTLS-Deep data are significantly deeper than the AEGIS ACS images. The CANDELS WFC3 data have just become available, and they are incorporated in the SED analysis. The possible contributors to the FIR sources remain unchanged.

\subsection{EGSO7 (EGS-J141900.3+524948)}

\subsubsection{Morphologies and Potential Components}

Figure 20 shows the images of EGS07. Within 18" of the $250 \mu \mathrm{m}$ source centroid, there are 55 objects detected in the CFHTLS Deep data that have $\mathrm{S} / \mathrm{N} \geqslant 5$ in the $i$ band (measured in the MAG_ISO aperture). Our inspection identified eight objects as the potential contributors to the $250 \mu \mathrm{m}$ flux, which we label as objects " 1 " to " 8 " in increasing order of distance to the $250 \mu \mathrm{m}$ source centroid. To emphasize the choice of the ground-based images for the counterpart identification, the labeling scheme here is numerical instead of alphabetical. In the $24 \mu \mathrm{m}$ image, these objects are all blended together as one single source and cannot be separated. This is also true in the 100 and $160 \mu \mathrm{m}$ images.

The central source, 1 , has spectroscopic redshift of $z=1.497$ from the DEEP2 galaxy redshift survey (Newman et al. 2013). In the ACS images, this source shows a very disturbed morphology that is indicative of strong interaction. This suggests that it could be the dominant contributor. Running SExtractor on the $I_{814}$ image, this source is broken into five components extending over $\sim 4$ ". 0 , for which we label as 1 -a to 1 -e (see Figure 21). The component $1-e$ is compact and consistent with being pointlike, and the others are all diffuse and irregular in shape. While they cannot be separated by SExtractor on the CFHTLS Deep images, most of these components are still visually discernible in these images with the exception that 1-e is swamped by 1-a due to the coarser spatial resolution and thus cannot be separated. As the CFHTLS data are deeper than the ACS images, 1-b is better detected (so is 2). The centroids of the 250 and $160 \mu \mathrm{m}$ images are between $1-\mathrm{a}, 1-\mathrm{d}$, and $1-\mathrm{e}$, while the $100 \mu \mathrm{m}$ centroid is $\sim 2$.'5 to the north.

Other objects do not have obvious features. 4, 5, and 8 are compact and point-like, while 2, 3, 6, and 7 are extended.

\subsubsection{Optical-to-near-IR SED Analysis}

The optical-to-near-IR SEDs were constructed using the CFHTLS-Deep $u^{*}$ griz and the CANDELS WFC $3 J_{125}$ and $H_{160}$ data, following the same procedures as in Section 5.1.2. The AEGIS ACS data were not used for this purpose because they are not as deep as the CFHTLS data. Unfortunately, the TFIT procedure using the WFC3 $H_{160}$ does not produce satisfactory results in IRAC (with bad residual maps) due to some unknown reasons, and therefore we had to exclude the IRAC data in this analysis.

The results are summarized in Figure 22. Object 1 has $z_{\mathrm{ph}}=1.46$, which agrees with its $z_{\mathrm{spec}}=1.497$ very well $(\Delta z /(1+z)=0.01)$. Object 6 has $z_{\mathrm{ph}}=1.48$, and from the $P(z)$ distribution it seems very likely that it is at the same redshift as 1. They are 3".4 apart, corresponding to $28.9 \mathrm{kpc}$, and hence could be in the same group. Their physical properties are rather different, however. 1 has a high stellar mass of $6.9 \times 10^{10} M_{\odot}$, a moderate age of $724 \mathrm{Myr}$, and an extended SFH with $\tau=7 \mathrm{Gyr}$ (i.e., almost constantly star forming as compared to its age). The inferred current SFR is $137 M_{\odot} \mathrm{yr}^{-1} .6$, on the other hand, is significantly less massive $\left(M^{*}=8.3 \times 10^{9} M_{\odot}\right)$ and much younger ( $T=182 \mathrm{Myr})$, and has assembled most of its current stellar mass through a short, modest episode of star formation ( $\tau=60 \mathrm{Myr}$ ) that leaves the current SFR of $\sim 10 M_{\odot} \mathrm{yr}^{-1}$.

Another possible group consists of objects 2, 4, 5, and 8, which have $z_{\mathrm{ph}}=1.01 \pm 0.07$. In terms of stellar mass, this group is not significant, as the most massive one, 5 , has only $M^{*}=3.2 \times 10^{9} M_{\odot}$.

Object 3 and 7 have $z_{\mathrm{ph}}=1.23$ and 1.89 , respectively, and thus are unlikely associated with either of the two possible groups.

\subsubsection{Decomposition in Mid-to-far-IR}

In $24 \mu \mathrm{m}$, the automatically iterative fit settled on objects 1,2 , and 3 . These three objects account for $\sim 61 \%, 32 \%$, and $6 \%$ of the total flux, respectively. For $70 \mu \mathrm{m}$, it converged on only 1 , although this leaves visible residual. In $100 \mu \mathrm{m}$, 1 was still the dominant component and account for $\sim 67 \%$ of the 

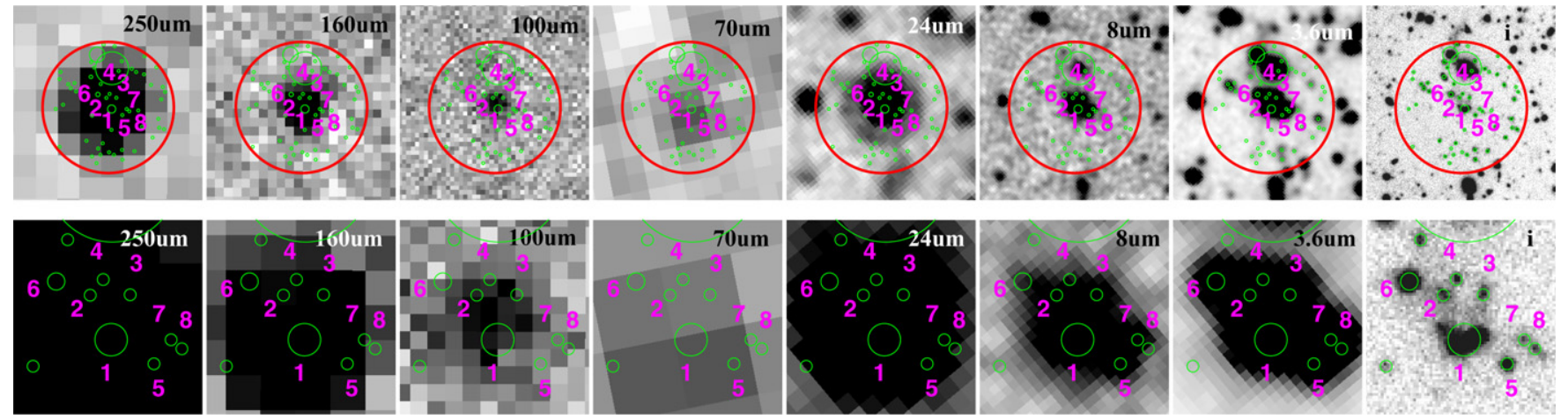

Figure 20. FIR to optical images of EGS07. The legends and the organization of the panels are the same as in Figure 2. The $i$-band image is from CFHTLS-Deep. (A color version of this figure is available in the online journal.)
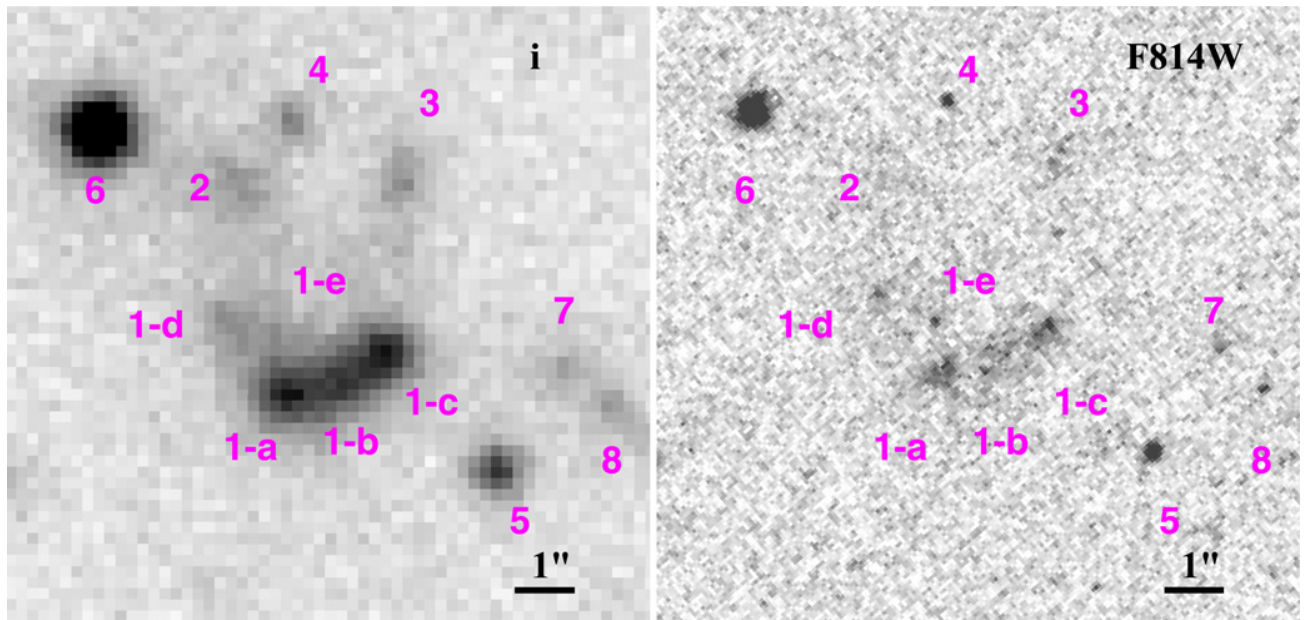

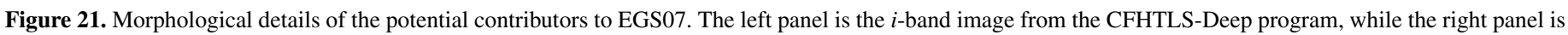
the $I_{814}$-band image from the AEGIS ACS program. The CFHTLS images are deeper that the ACS images, but the latter show more morphological details.

(A color version of this figure is available in the online journal.)

total flux, however, the secondary components changed to 4 and 6 , which are responsible for $15 \%$ and $17 \%$ of the total flux, respectively. In $160 \mu \mathrm{m}$, the automatically iterative fit failed, and we had to use the trial-and-error method. The only sensible results were obtained when the fit used 1 and 4 or just 1, and we adopted the former case because 4 seems to be non-negligible in $100 \mu \mathrm{m}$, although this reason is not overwhelming. The flux density of 1 would increase by $\sim 10 \%$ if we were to choose otherwise. Similarly, we had to use the trial-and-error approach to fit $250 \mu \mathrm{m}$, and the only sensible results were obtained when using 1 and 4 or just 1 , and we again adopted the former case. If we were to chose otherwise, the flux density of 1 would increase by $24 \%$. The decomposition in $250 \mu \mathrm{m}$ in demonstrated in Figure 23. Regardless of the exact counterparts in each band, it is obvious that 1 is the dominant contributor to the mid-to-farIR emission. The flux densities of this object are summarized in Table 2.

\subsubsection{Total IR Emission and Stellar Populations}

Figure 24 summarizes the analysis of the FIR emission of EGS07-1. As it turns out, the SK07 models can provide a good fit from $70 \mu \mathrm{m}$ to $250 \mu \mathrm{m}$, but the $24 \mu \mathrm{m}$ data point cannot be satisfactorily explained (left panel of Figure 24). Nevertheless, the derived $L_{\mathrm{IR}}=4.0 \times 10^{12} L_{\odot}$ agrees reasonably well with $L_{\mathrm{IR}}=5.0 \times 10^{12} L_{\odot}$ inferred from the analytic fit (right panel), and hence it seems robust that this object is a ULIRG.
Object 1 has an X-ray counterpart in the $800 \mathrm{Ks}$ AEGIS Chandra data (K. Nandra et al., in preparation; Laird et al. 2009), ${ }^{16}$ with the full-band fluxes in $0.5-10 \mathrm{keV}$ of $7.05_{-1.37}^{+1.55} \times$ $10^{-16} \mathrm{erg} \mathrm{s}^{-1} \mathrm{~cm}^{-2}$. At $z=1.497$, this implies a total X-ray luminosity in rest-frame $1.2-25 \mathrm{keV}$ of $1.0 \times 10^{43} \mathrm{erg} \mathrm{s}^{-1}$, which is in the AGN regime. Thus the FIR emission of 1 might have an AGN contribution, which might be the reason that the $24 \mu \mathrm{m}$ data point is not well fitted. Its X-ray position has a very good accuracy of 0.56 , and is consistent with the sub-component 1-e. Among all the sub-components of 1,1 -e is unique in its pointlike morphology and its absence from the $V_{606}$-band. This raises a possibility that 1 -e might not be part of 1 but might actually be a background quasar, which could be at $z \sim 5$ given the fact that it is a dropout from $V_{606}$. If this is true, its X-ray luminosity would be $4.9 \times 10^{44} \mathrm{erg} \mathrm{s}^{-1}$. However, the FIR emission of EGS07 would not be from 1-e alone, otherwise the SPIRE flux densities would peak at $500 \mu \mathrm{m}$ instead of between 250 and $350 \mu \mathrm{m}$. Estimating the IR luminosity for $1-\mathrm{e}$ only is hardly possible in this case, as 1-e is so close to other subcomponents that it cannot be decomposed in the FIR. Therefore, while it is clear that 1 has an AGN and that the AGN could contribute significantly to the FIR emission, the nature of object 1 remains inconclusive at this point. Taking $L_{\mathrm{IR}}$ at its face value, one would

\footnotetext{
16 Object 5 also has an X-ray counterpart that has the full-band $0.5-10 \mathrm{keV}$ fluxes of $12.78_{-1.79}^{+1.95} \times 10^{-16} \mathrm{erg} \mathrm{s}^{-1} \mathrm{~cm}^{-2}$. However, we do not discuss it further as it does not seem to be a significant contributor to the FIR flux.
} 

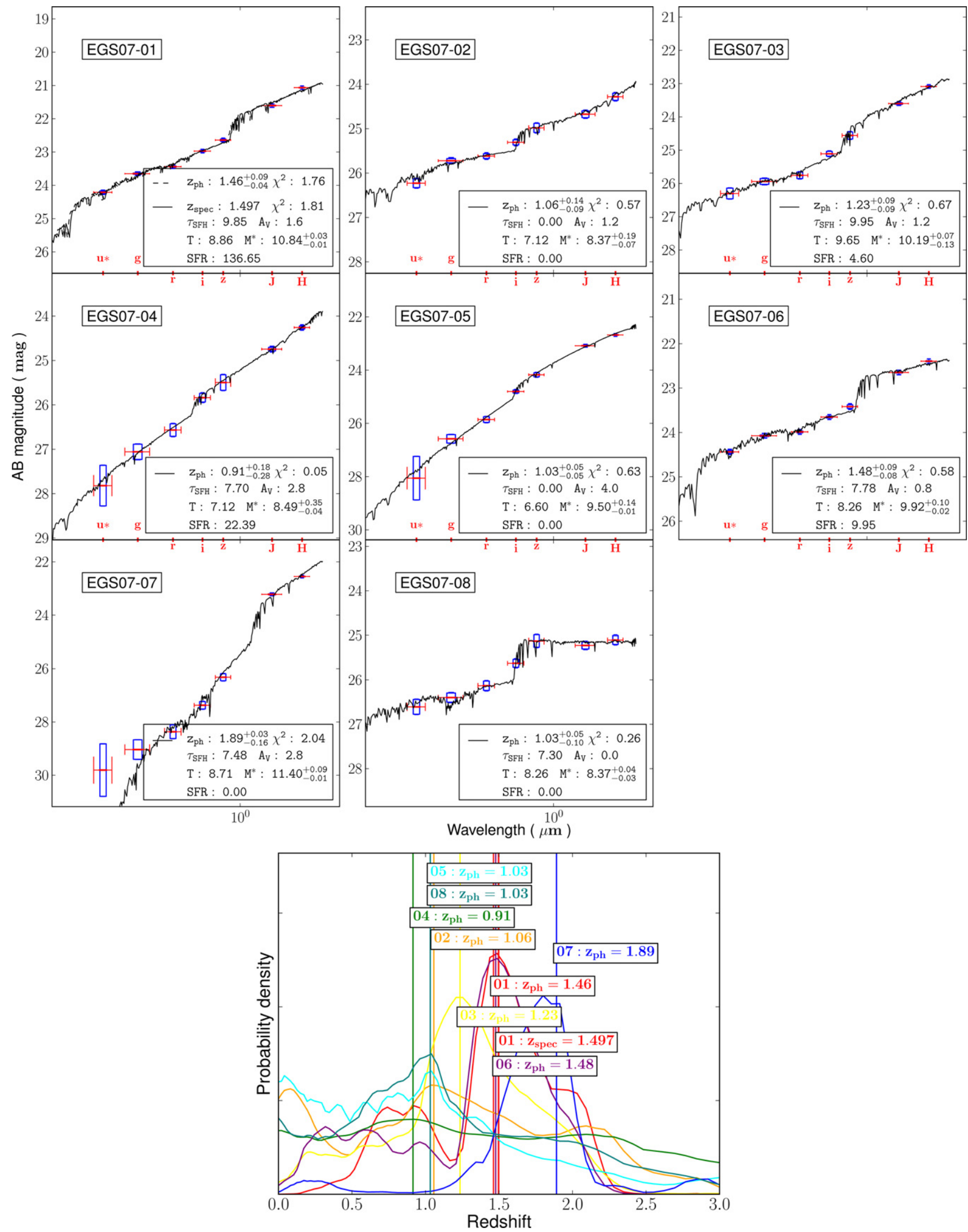

Figure 22. Optical-to-NIR SED analysis of the possible contributors to EGS07. Legends are the same as in Figure 4. Objects 1 and 6 are likely at the same redshift. Another possible group consists of 2, 4, 5, and 8 at $z_{\mathrm{ph}}=1.01$.

(A color version of this figure is available in the online journal.) 

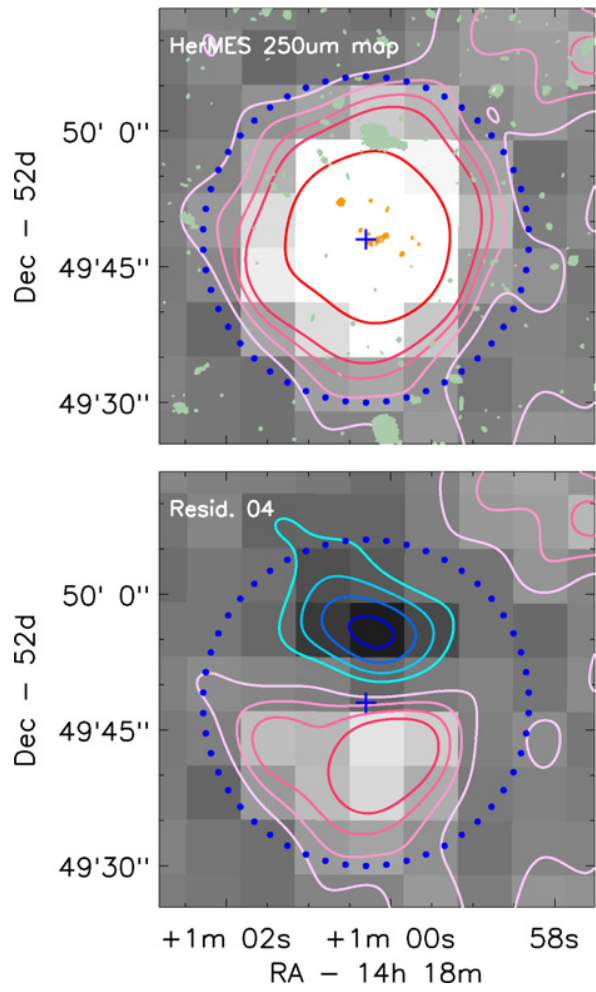

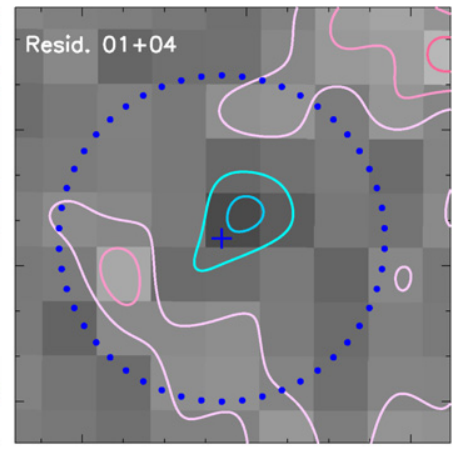

$+1 \mathrm{~m} \mathrm{02s}+1 \mathrm{~m} \mathrm{00s}$

RA $-14 \mathrm{~h} 18 \mathrm{~m}$

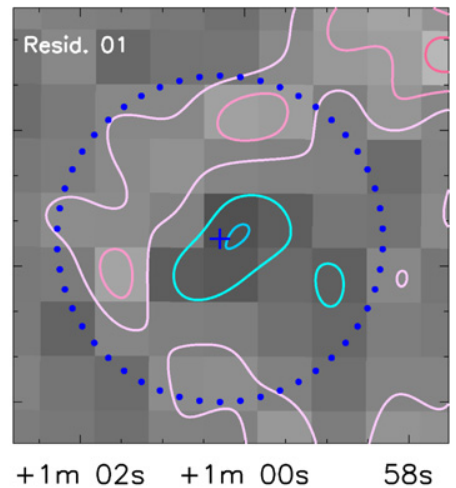

RA - $14 \mathrm{~h} 18 \mathrm{~m}$

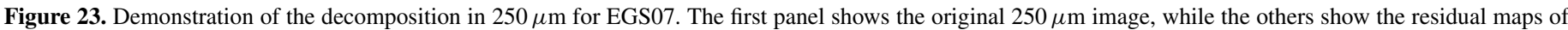

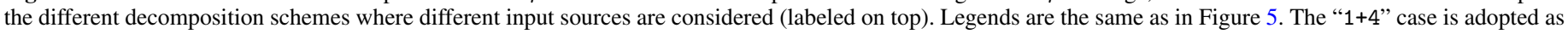
the final solution. See text for details.

(A color version of this figure is available in the online journal.)
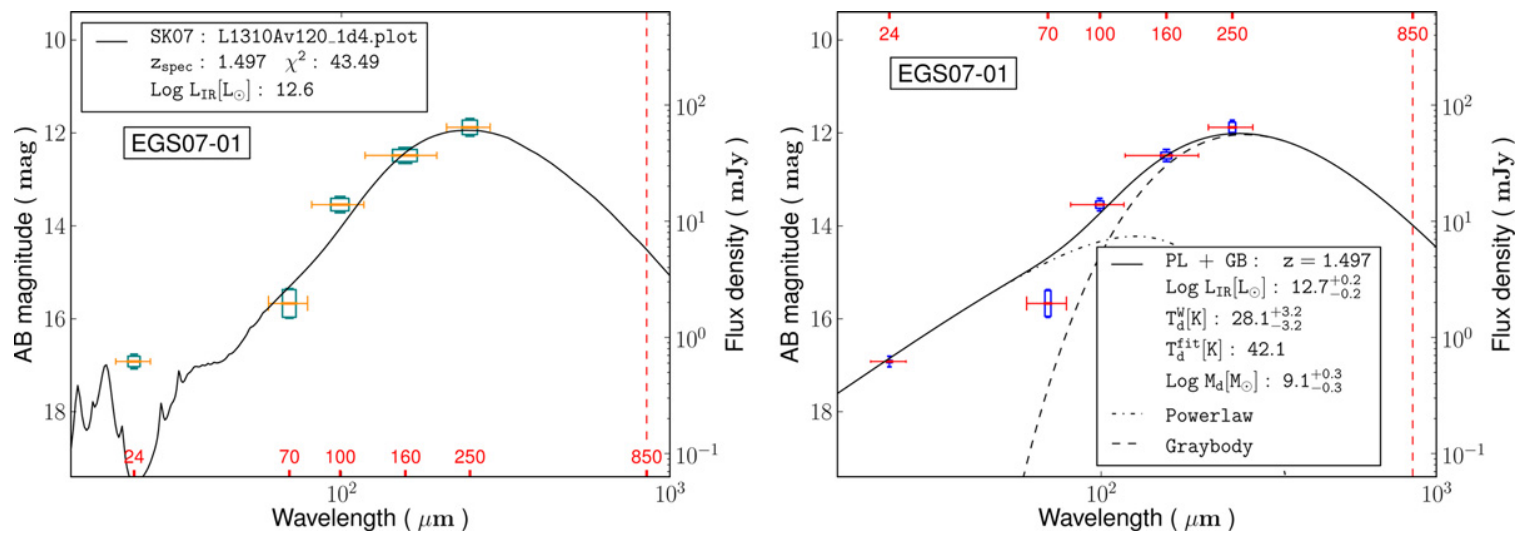

Figure 24. Mid-to-FIR SED analysis for EGS07. Legends are the same as Figure 6 . The $24 \mu \mathrm{m}$ data point cannot be well explained by the best-fit SK07 models (left panel). Nevertheless, the derived $L_{\mathrm{IR}}$ is in good agreement with the result from the fit to the power-law + graybody models (right panel).

(A color version of this figure is available in the online journal.)

infer an upper limit of $\mathrm{SFR}_{\mathrm{IR}}=400 M_{\odot} \mathrm{yr}^{-1}$. We refrain from discussing the SFR of this system in the usual way.

Finally, we investigate the FIR-radio relation for this source. The catalog of Ivison et al. (2010) includes a strong radio source at the position of $1-\mathrm{e}$, which has $S_{1.4 \mathrm{GHz}}=0.316 \mathrm{mJy}$. We obtained $q_{\mathrm{IR}}=2.02$, which is significantly lower than $2.40 \pm 0.24$.

\subsection{EGS14 (EGS-J142025.9+525935)}

\subsubsection{Morphologies and Potential Components}

Figure 25 shows the images of EGS14. Within $18^{\prime \prime}$ of the $250 \mu \mathrm{m}$ source centroid, there are 58 objects detected in $i$ that have $S / N \geqslant 5$. Based on inspection, we identified 10 objects as potential contributors to the $250 \mu \mathrm{m}$ flux, which we label as "1" to "10." In this area, only two sources are discernible in the $24 \mu \mathrm{m}$ image, which seem to be dominated by 5 and 9 , respectively. While it is difficult to see from the 100 and $160 \mu \mathrm{m}$ images, the PEP DR1 catalog identifies two sources in this area as well, and their positions are also consistent with 5 and 9 , respectively. All this suggests that 5 and 9 are the major contributors to the $250 \mu \mathrm{m}$ flux.

The ACS images reveal that both 5 and 9 have interesting morphologies (Figure 26). They are 4".39 and 5"'67 from the $250 \mu \mathrm{m}$ centroid, respectively, and are on the opposite sides (10'0 apart). 5 is a very disturbed, curvy, and knotty system whose shape resembles a scorpion. 9 is also highly irregular, having a bright central core and at least two satellite features 

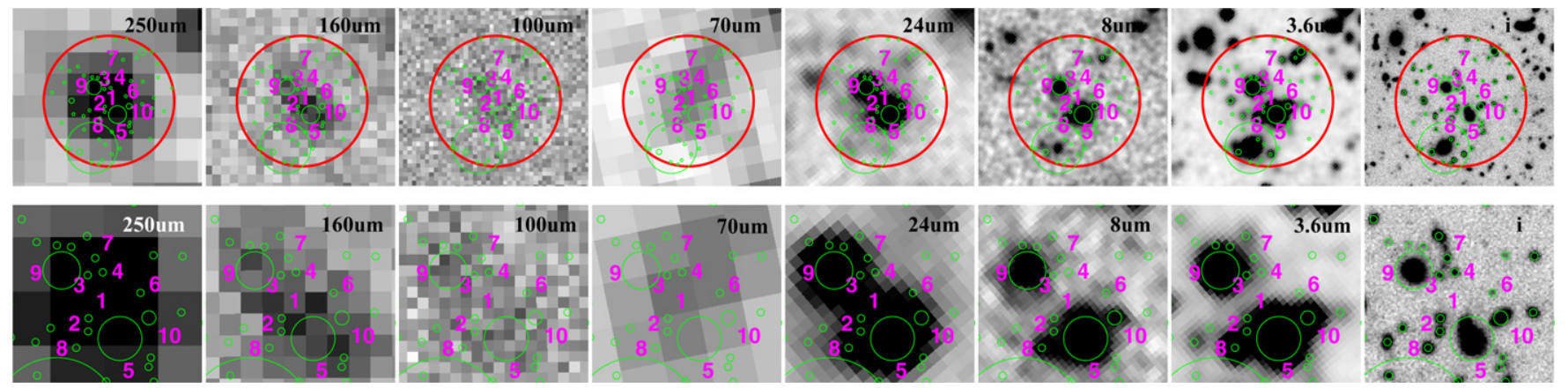

Figure 25. FIR to optical images of EGS14. The legends and the organization of the panels are the same as in Figure 2. The $i$-band image is from CFHTLS-Deep. (A color version of this figure is available in the online journal.)
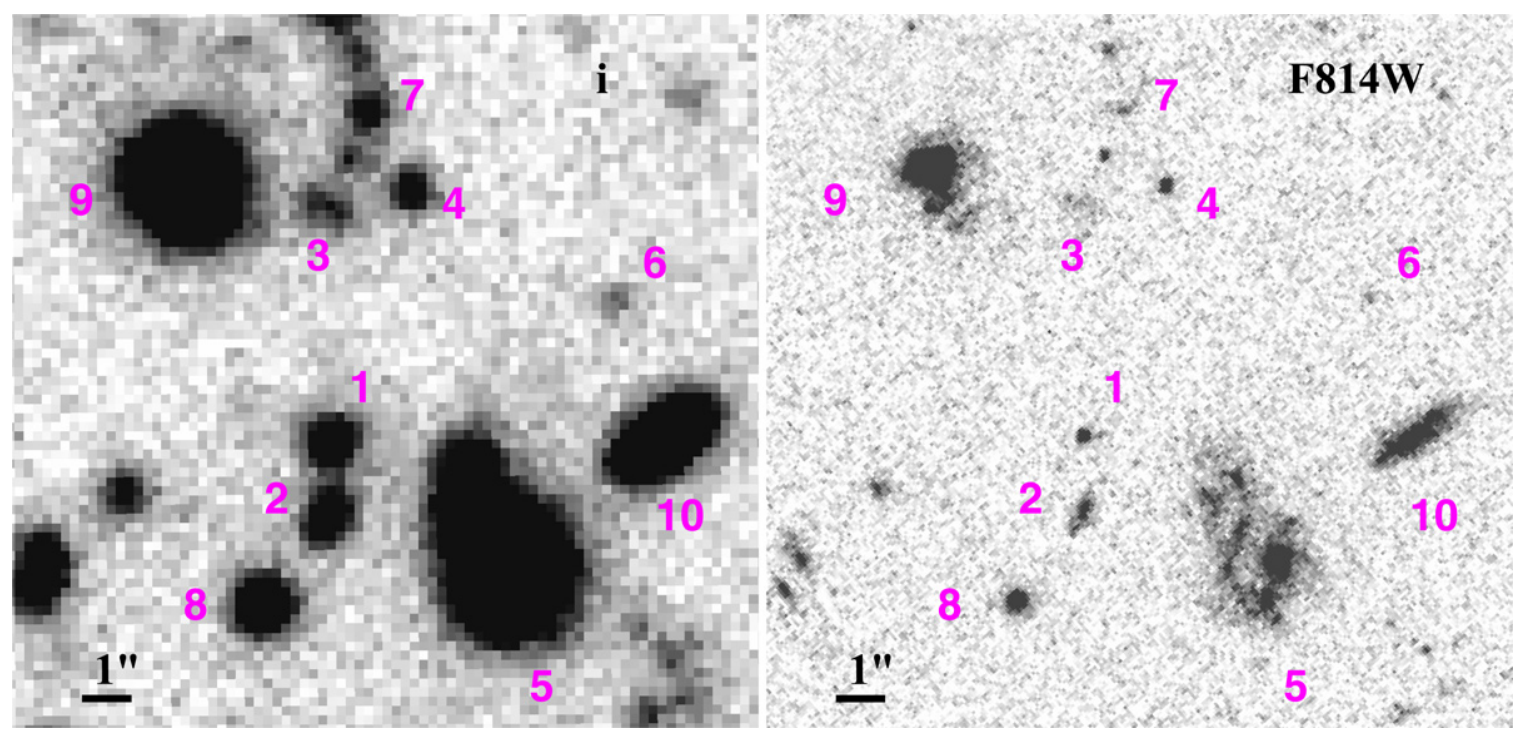

Figure 26. Morphological details of the potential contributors to EGS14 in the $i$ band from CFHTLS-Deep (left) and in the $I_{814}$ band from the AEGIS ACS (right). (A color version of this figure is available in the online journal.)

around it. Among the other objects, 1, 4, and 8 are compact, and the rest are extended. 2 and 10 seem to be disk systems with dust lanes, 3 and 6 are amorphous, and 7 actually consists of one central core and two smaller objects on each side.

\subsubsection{Optical-to-near-IR SED Analysis}

None of these 10 objects has spectroscopic redshifts, and we derived their $z_{\mathrm{ph}}$ in the usual way. The results are summarized in Figure 27. The optical-to-near-IR SED are different from those in Section 6.1.2 in that we are able to incorporate the SEDS IRAC 3.6 and $4.5 \mu \mathrm{m}$ data, as the TFIT procedure produces good results comparable to those in Sections 4 and 5. Object 6 has to be excluded, as it is only significantly detected in $r$ and $i$. This leaves nine objects in total. The fits to objects 1,3 , and 7 are poor, but these three sources are likely irrelevant to the FIR emission (see below in Section 6.2.3). Most of these objects are likely at $z \approx 1$. In particular, objects 5 and 9 have their $z_{\text {ph }}$ agrees extremely well, at 1.11 and 1.12 , respectively. 2,4 , and 8 all have $\Delta z /(1+z) \leqslant 0.05$ with respect to 5 and 9 , and the peaks of their $P(z)$ distributions coincide well with each other. This suggests that EGS14 could be in a rich group environment. Objects 5 and 9 are by far the most dominant members of this possible group. However, their stellar populations are distinctly different. 5 has a very high stellar mass of $3.0 \times 10^{11} M_{\odot}$ and an old age of $5.5 \mathrm{Gyr}$, comparable to the age of the universe at this redshift. It has a prolonged SFH with $\tau=4.0 \mathrm{Gyr}$, which is comparable to its age, and a modest ongoing $\mathrm{SFR}=45.5 M_{\odot} \mathrm{yr}^{-1}$. If 5 indeed is a merging system, as its morphology suggests, its subcomponents must have been persistently and gradually assembling their stellar masses right after the big bang, at a nearly constant rate of $\sim 50 M_{\odot} \mathrm{yr}^{-1}$ (combining over all its subcomponents). Using the conversion from SFR to UV luminosity as in Madau et al. (1998), one can see that the progenitor of this entire system would be quite readily visible by $z \approx 10$, with a total magnitude of $H_{A B} \approx 25.2$ if there were not much dust at such an early stage. 9 , on the other hand, has a much lower stellar mass of $5.0 \times 10^{10} M_{\odot}$ and a young age of $129 \mathrm{Myr}$. Its SFH is an intense, short burst with $\tau=40 \mathrm{Myr}$, which still leaves an ongoing SFR $=72 M_{\odot} \mathrm{yr}^{-1}$.

\subsubsection{Decomposition in Mid-to-far-IR}

The decomposition is carried out for the nine objects using their centroids determined from the CFHTLS-Deep $i$-band.

The automatically iterative fit succeeded in the $24 \mu \mathrm{m}$ and $250 \mu \mathrm{m}$, the latter of which is shown in Figure 28 for demonstration. For the $24 \mu \mathrm{m}$, the fit converged on 2, 5, 9, and 10. Objects 5 and 9 almost equally split $\sim 82 \%$ of the total light in this area. For the $250 \mu \mathrm{m}$, the fit converged on 5, 7, 8, 9, and 10. However 7,8 , and 10 were formally rejected because they all had negligible fluxes, and hence the output only included 5 and 9 . Fitting only 5 or only 9 results in obvious residuals at the position of the other object, and hence is not acceptable. 

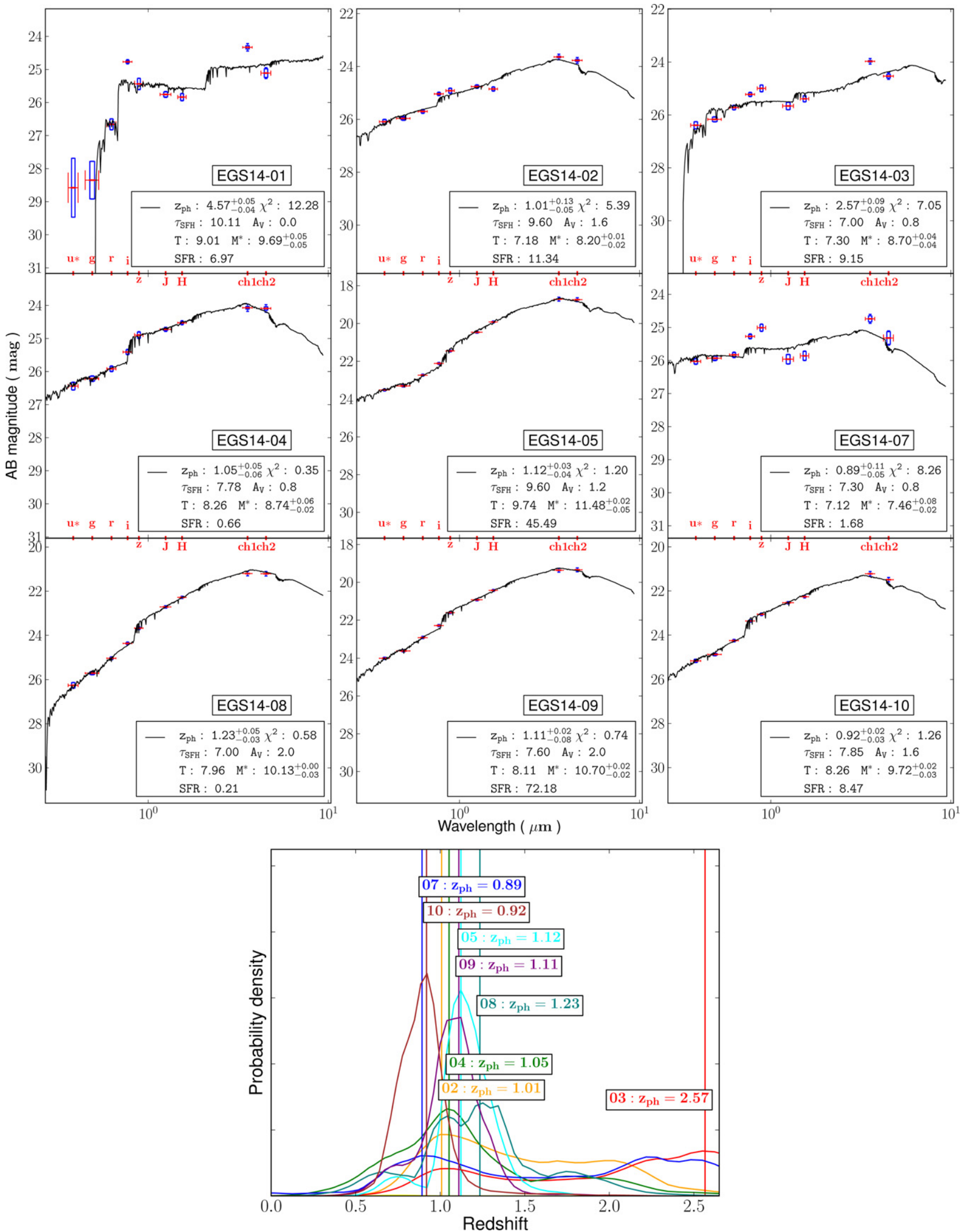

Figure 27. Optical-to-NIR SED fitting results for EGS14. Legends are the same as in Figure 4. The two most massive objects, 5 and 9 , are likely at the same redshift $\left(z_{\mathrm{ph}}=1.11-1.12\right)$ and associated; objects 2,4 , and 8 could also belong to this group.

(A color version of this figure is available in the online journal.) 

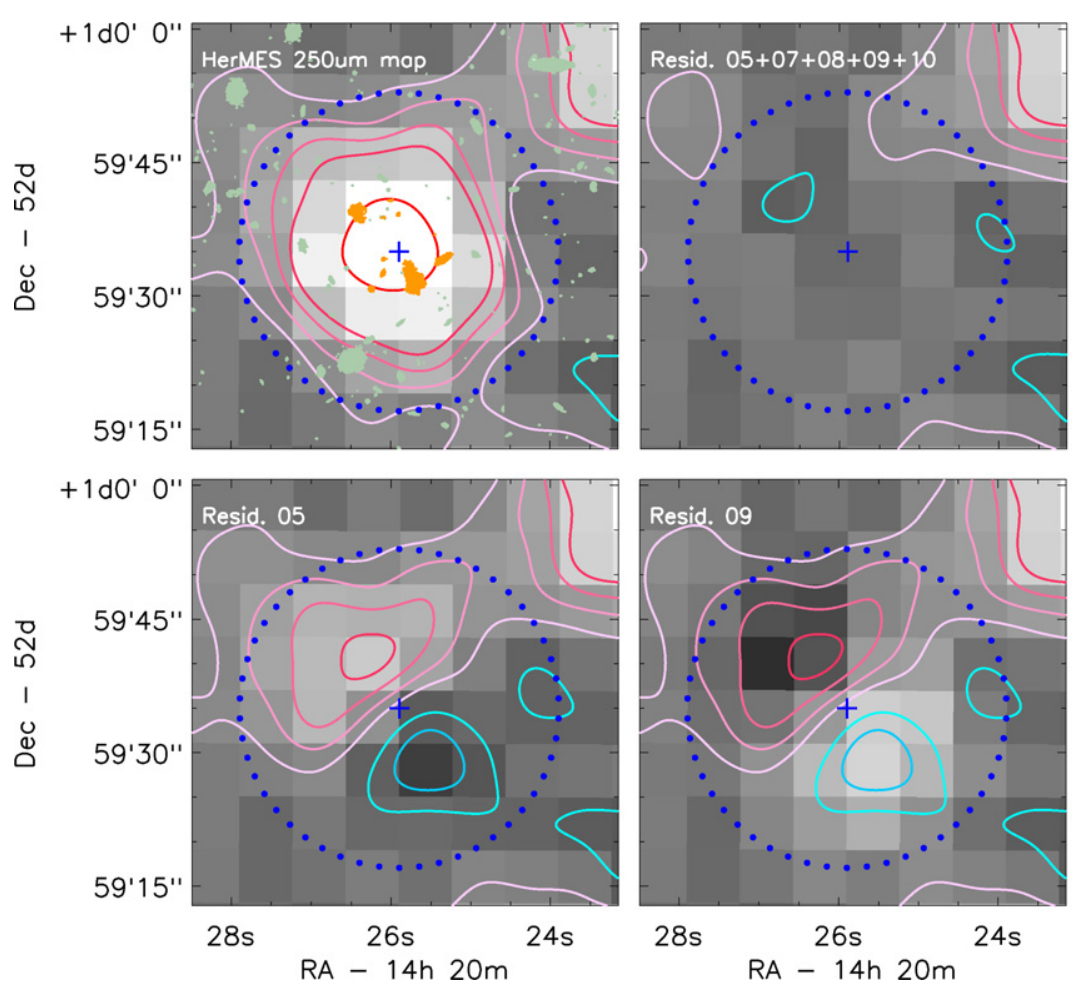

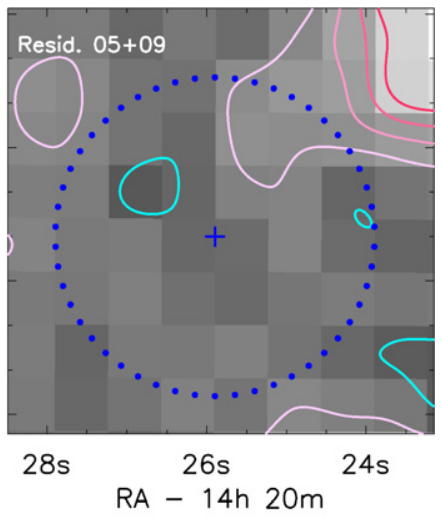

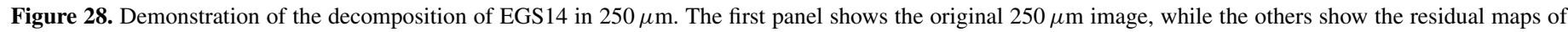

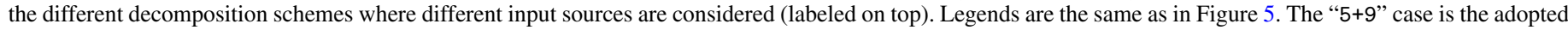
final solution.

(A color version of this figure is available in the online journal.)

Unfortunately, the decomposition in $70 \mu \mathrm{m}$ using either the automatically iterative method or the trail-and-error method could not converge on any object, apparently because of the low $\mathrm{S} / \mathrm{N}$ of the data. Therefore we had to skip this band. The decomposition in both 100 and $160 \mu \mathrm{m}$ also failed due to the low $\mathrm{S} / \mathrm{N}$ of the data. Nevertheless, the PEP DR1 catalog includes two sources whose position coincide with 5 and 9 . As the decomposition of both the $24 \mu \mathrm{m}$ and the $250 \mu \mathrm{m}$ images shows that 5 and 9 are by far the most dominant objects in both bands, we adopted the PEP catalog values for these two objects in 100 and $160 \mu \mathrm{m}$ and assumed that all other objects are negligible in these two bands. Table 2 summarizes these results for the major components 5 and 9.

\subsubsection{Total IR Emission and Stellar Populations}

The FIR emission of EGS14 should be mostly from 5 and 9. Based on their $z_{\mathrm{ph}}$ estimates, we adopted $z_{\mathrm{ph}}=1.12$ as their common redshift. Based on the AEGIS X-ray catalog, there is no X-ray source detected in the EGS14 region. The limit of this catalog in the most sensitive $0.5-2 \mathrm{keV}$ band is $\sim 3 \times 10^{-17} \mathrm{erg} \mathrm{s}^{-1} \mathrm{~cm}^{-2}$, which corresponds to an upper limit of $2.1 \times 10^{41} \mathrm{erg} \mathrm{s}^{-1}$ in the rest-frame $1-4 \mathrm{keV}$ at $z=1.12$. Therefore, we believe that its FIR emission is most likely due to star formation. Fitting their mid-to-far-IR SED separately to the SK07 models, we got $L_{\mathrm{IR}}=1.3 \times 10^{12}$ and $1.0 \times 10^{12} L_{\odot}$ for 5 and 9, respectively, and therefore they are both ULIRG. This is shown in the left panels of Figure 29. From the results in Section 6.2.2, we also got $L_{\mathrm{IR}}^{\mathrm{ext}}=4.7 \times 10^{11} L_{\odot}$ for 5 , which then implies $L_{\mathrm{IR}}^{\mathrm{blk}}=8.3 \times 10^{11} L_{\odot}$ and $\mathrm{SFR}_{\mathrm{IR}}^{\mathrm{blk}}=83 M_{\odot} \mathrm{yr}^{-1}$ for this object. It has $\mathrm{SFR}_{\text {fit }}=46 M_{\odot} \mathrm{yr}^{-1}$, and hence we get $\mathrm{SFR}_{\text {tot }}=129 M_{\odot} \mathrm{yr}^{-1}$. For 9 , we got $L_{\mathrm{IR}}^{\mathrm{blk}}=1.2 \times 10^{12} L_{\odot}$, which is even slightly larger than $L_{\mathrm{IR}}$. In this case, we take it that the observed IR emission can be fully explained by the extinction in the exposed region.

From the analytic fits, we got $T_{d}^{\mathrm{fit}}=36.9$ and $37.2 \mathrm{~K}$, and $M_{d}=4.0 \times 10^{8}$ and $3.2 \times 10^{8} M_{\odot}$ for 5 and 9 , respectively. All this suggests that the dust properties of these two ULIRGs are very similar. However, as discussed in Section 6.2.2, the stellar populations in the exposed regions of these two objects are vastly different in their stellar masses, ages, and SFHs: 5 has $M^{*}=3.0 \times 10^{11} M_{\odot}, T=5.5 \mathrm{Gyr}$, and $\tau=4.0 \mathrm{Gyr}$, while 9 has $M^{*}=5.0 \times 10^{10} M_{\odot}, T=129 \mathrm{Myr}$, and $\tau=129 \mathrm{Myr}$. Therefore, these imply SSFR $=0.4$ and $1.4 \mathrm{Gyr}^{-1}$ for 5 and 9 , respectively. For 5 , one can also get $T_{\mathrm{db}}^{\text {tot }}=2.3 \mathrm{Gyr}$ and $T_{\mathrm{db}}^{\mathrm{blk}}=3.6$ Gyr. For $9, T_{\mathrm{db}}^{\mathrm{tot}}=694 \mathrm{Myr}$, and $T_{\mathrm{db}}^{\mathrm{blk}}$ is not applicable. The inferred total gas masses for these two objects from the above dust masses are $5.6 \times 10^{10} M_{\odot}$ and $4.4 \times 10^{10} M_{\odot}$, respectively. Therefore, the gas reservoir for 5 would only allow it to add $<20 \%$ to its existing stellar mass even if it could turn all the available gas into stars. On the other hand, the situation for 9 is somewhat different in that its current ULIRG phase would be able to add $\sim 88 \%$ to its existing stellar mass if it could turn all the gas into stars, however, this would require a much longer time than the typical lifetime of a ULIRG. Considering that its ongoing star formation is all in the exposed region and has a sharp declining SFH, this is not likely to happen.

Finally we investigate the FIR-radio correlation for EGS14. The catalog of Ivison et al. (2010) does not include any strong radio source in the area of EGS-14. However, from the radio map (R. J. Ivison 2013, private communication) we detect two moderate sources at the exact locations of 5 and 9, which have $S_{1.4 \mathrm{GHz}}=0.043 \pm 0.012$ and $0.071 \pm 0.013 \mathrm{mJy}$, respectively. From these we obtained $q_{\mathrm{IR}}=2.63$ and 2.34 , respectively, 

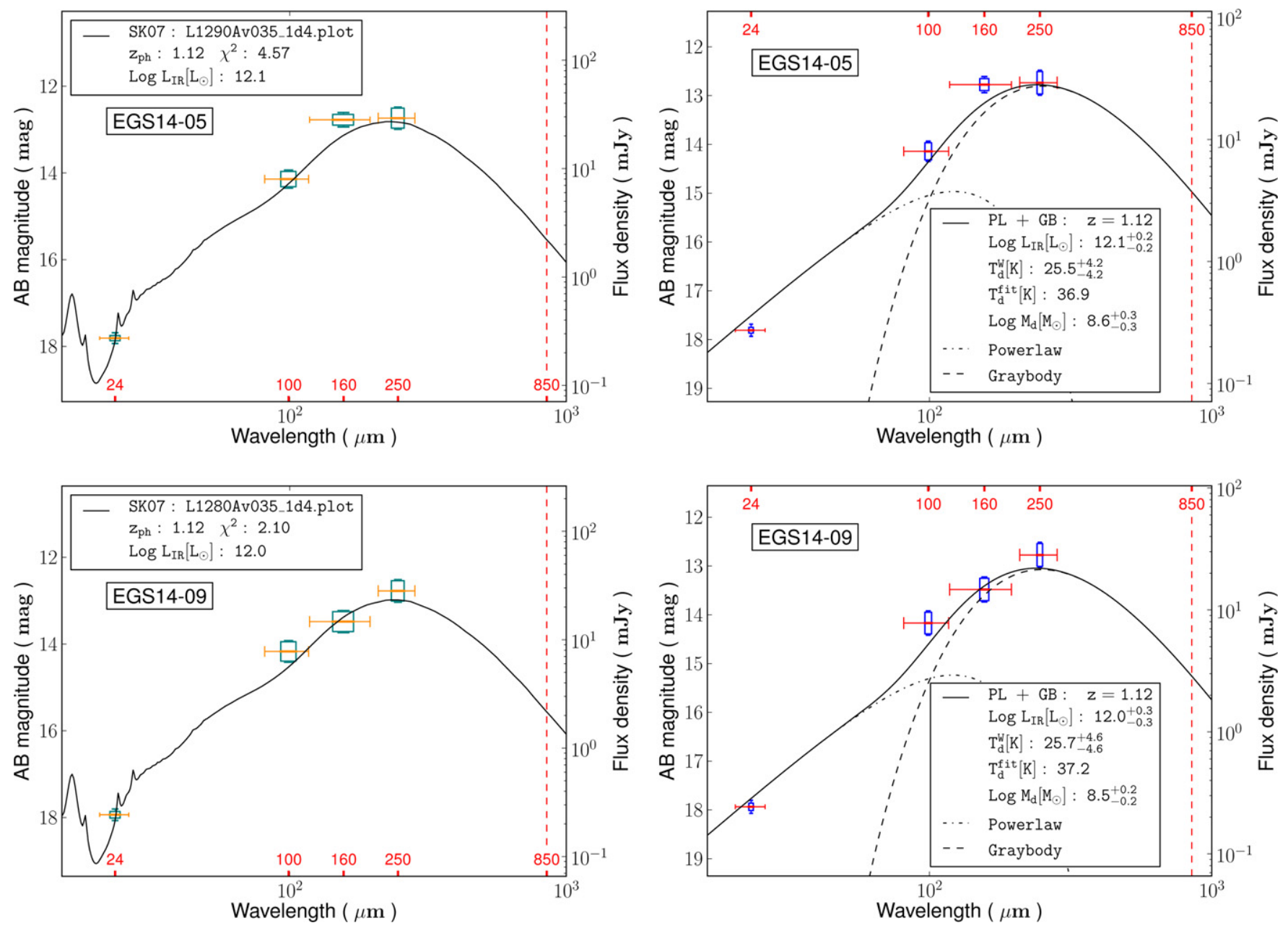

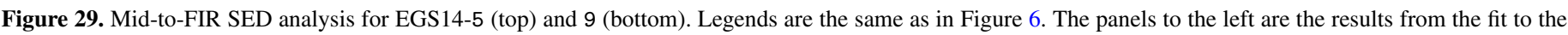
SK07 models, while the panels to the right are the results from the fit to the power-law + graybody models.

(A color version of this figure is available in the online journal.)
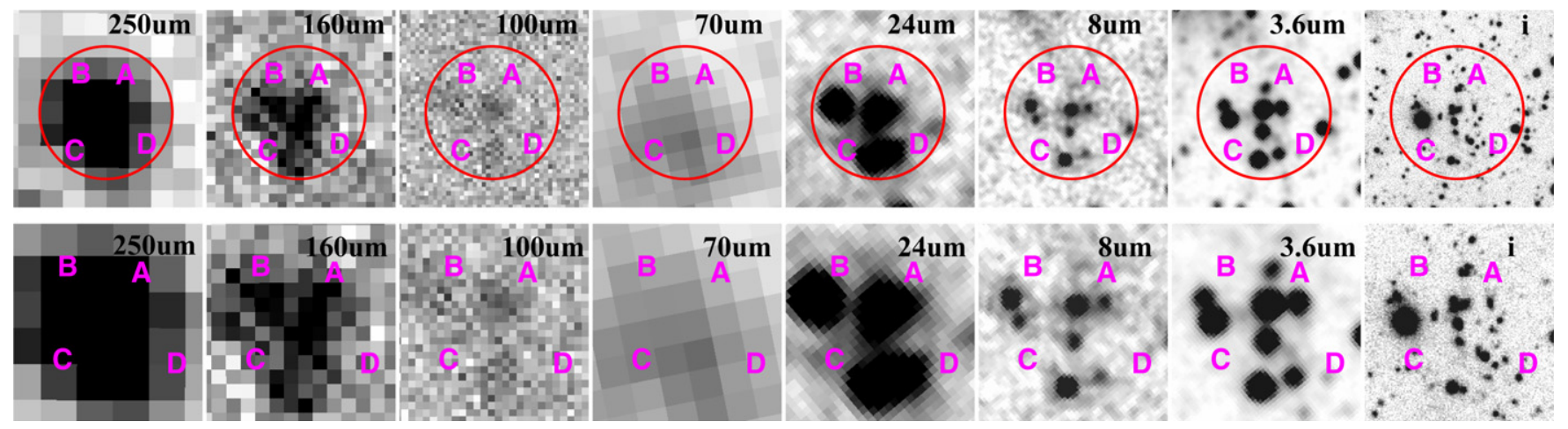

Figure 30. FIR to optical images of EGS19. The legends and the organization of the panels are the same as in Figure 2. The $i$-band image is from CFHTLS-Deep.

(A color version of this figure is available in the online journal.)

which are consistent with the mean of $2.40 \pm 0.24$ in Ivison et al. (2010).

\section{3. $\operatorname{EGS19}(E G S-J 141943.4+525857)$}

This source is different from the previous two in that it is outside of the CANDELS WFC3 area. At its $250 \mu \mathrm{m}$ source location, the PEP catalog reports three sources. From north to south, their flux densities are $6.4 \pm 1.2,9.9 \pm 1.2$, and $6.0 \pm 1.2 \mathrm{mJy}$ in $100 \mu \mathrm{m}$, respectively, and $19.7 \pm 3.3$, $22.0 \pm 3.4$, and $17.1 \pm 2.9 \mathrm{mJy}$, respectively.

\subsubsection{Morphologies}

Figure 30 shows the images of EGS-19. Within $r=18^{\prime \prime}$, there are 46 objects detected in the CFHTLS-Deep data that have $\mathrm{S} / \mathrm{N} \geqslant 5$ in the $i$-band measured in the MAG_ISO aperture. Among these objects, more than 20 of them could be possible contributors, most of which segregate into groups. The subcomponents within each group are so close to each other that using them directly for the decomposition would crash the process. Therefore, we took a slightly different approach in analyzing this source. To emphasize this difference, we use yet 


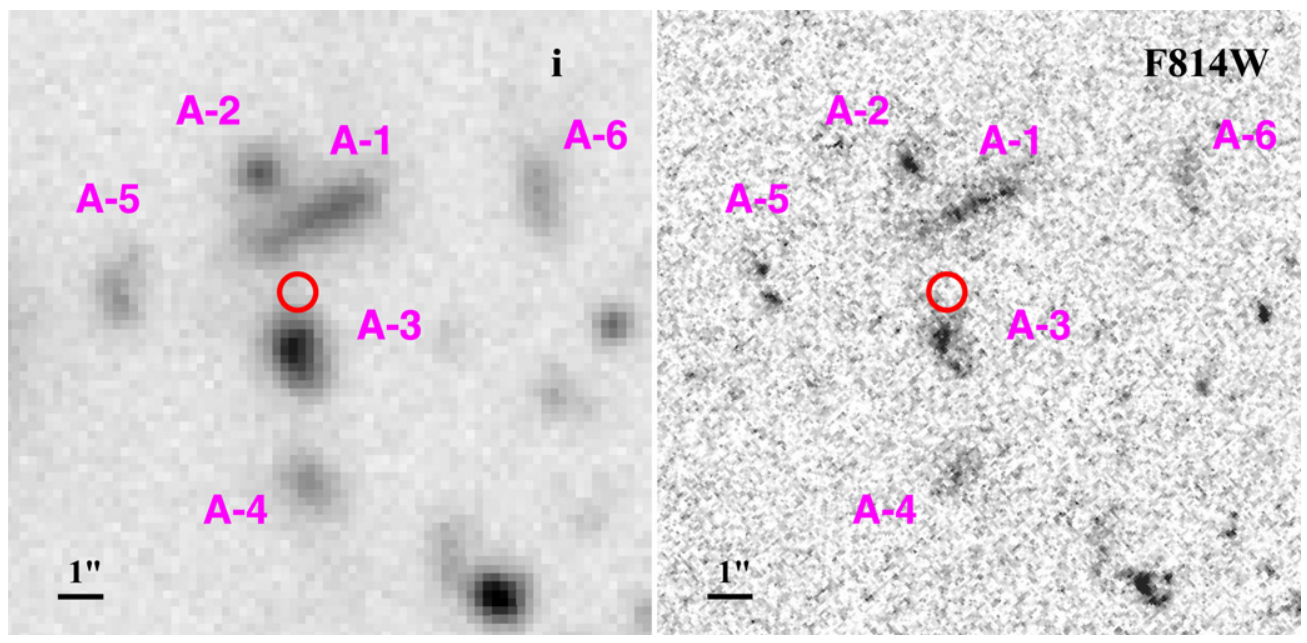

Figure 31. Morphological details of EGS-19, $\mathbb{A}$ component in the CFHTLS-Deep $i$ band (left) and the AEGIS $I_{814}$ band (right). The red circle is 0 ' 4 in radius and indicates the position of $\mathrm{A}^{\prime}$, which is the geometric center of $\mathbb{A}-1,2$, and 4 (see Section 6.3.2).

(A color version of this figure is available in the online journal.)

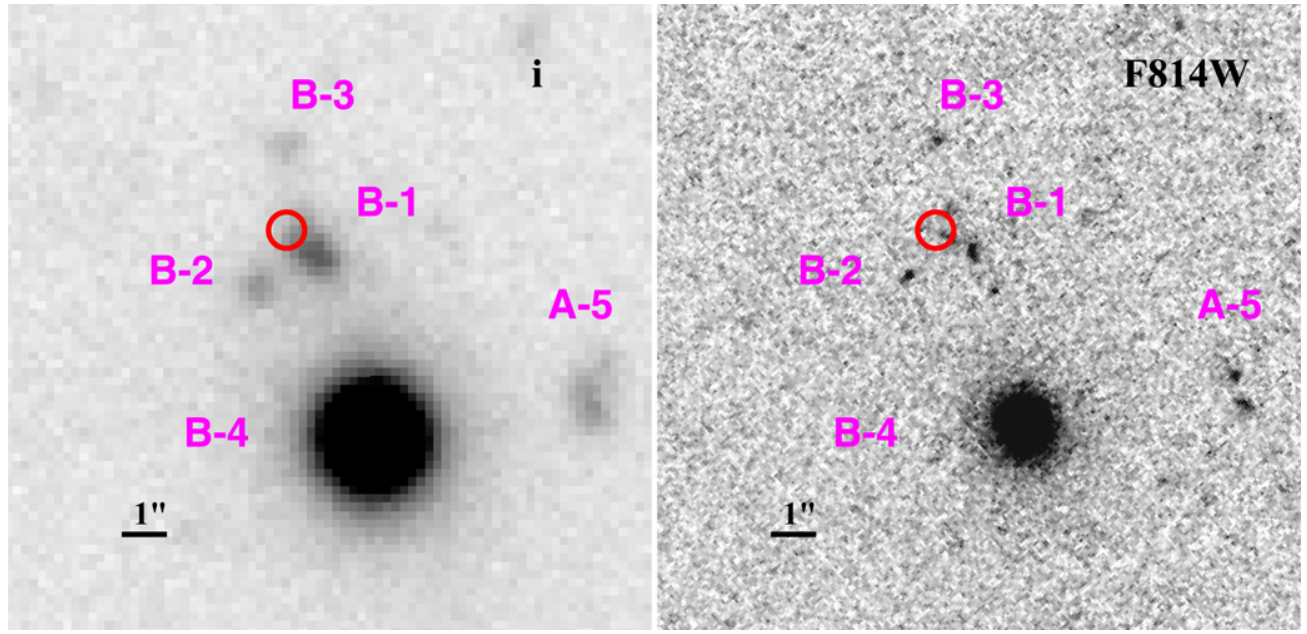

Figure 32. Similar to Figure 31, but for the $\mathbb{B}$ component of EGS19. The red circle indicates the position of $\mathrm{B}^{\prime}$, which is the geometric center of $\mathbb{B}-1,2$, and 3 (see Section 6.3.2)

(A color version of this figure is available in the online journal.)

another different labeling scheme in referring to the possible counterparts. From the $24 \mu \mathrm{m}$ image, we identified four possible contributors, which are labeled from $\mathbb{A}$ to $\mathbb{D}$. Each of these $24 \mu \mathrm{m}$ sources could be made of several components as revealed by the CFHTLS Deep images, for which we label numerically as shown in Figures 31-33.

Component $\mathbb{A}$ is made of six subcomponents. In the higher resolution ACS images, $\mathbb{A}-1$ seems like a dusty disk system viewed edge-on. $\mathbb{A}-2$, which is only $1^{\prime \prime} .56$ away from $\mathbb{A}-1$, is a small but resolved galaxy whose major axis is almost perpendicular to $\mathbb{A}-1$. $\mathbb{A}-3$ consists of two irregular objects separated by 0.69 , which seem to be embedded in a somewhat extended halo. $\mathbb{A}-4$ is an irregular object with a small core. $\mathbb{A}-5$ consists of two objects separated by 0.67 , one resolved and the other unresolved. $\mathbb{A}-6$ is irregular and does not have a well defined core.

Component $\mathbb{B}$ consists of four possible subcomponents. As revealed by the ACS images, $\mathbb{B}-1$, which is closest to the $24 \mu \mathrm{m}$ source centroid, is made of three irregular objects ${ }^{17}$ that

17 The additional, compact "object" to the southwest is actually due to bad pixels. stretch over $\sim 1^{\prime \prime}$. While currently we do not have any additional data to determine if these three objects are at the same redshift, we assume that they are associated and take them as one single object. $\mathbb{B}-2$ and $\mathbb{B}-3$ are two small, compact objects that are 2 . 3 and 4 .' 7 away from $\mathbb{B}-1$, respectively. $\mathbb{B}-4$ is a regular spheroidal. While it is the brightest in optical among all, it is far away from the $24 \mu \mathrm{m}$ source center $\left(\sim 4^{\prime \prime}\right)$ and likely only contributes a minimal amount to the $24 \mu \mathrm{m}$ flux.

Component $\mathbb{C}$ consists of two objects. $\mathbb{C}-1$ seems to be a regular elliptical in the CFHTLS images, however, the ACS images reveal that it is most likely a merger. It has a spectroscopic redshift of 1.180 from the Deep3 program. $\mathbb{C}-2$ is 1.9 away, and is a point source.

Component $\mathbb{D}$, which is 6.' 1 away from $\mathbb{C}-1$, is blended with $\mathbb{C}$ in $24 \mu \mathrm{m}$. The CFHTLS images show that it is made of two subcomponents separated by $\sim 0^{\prime} .5$. The surface brightness of $\mathbb{D}$ is quite low, and it is not detected in the AEGIS images.

\subsubsection{Optical SED Analysis and Redshifts}

To proceed with our analysis, the first step is to determine if the subcomponents in each of the four $24 \mu \mathrm{m}$ clumps could be at the same redshifts. As EGS-19 does not have WFC3 images 


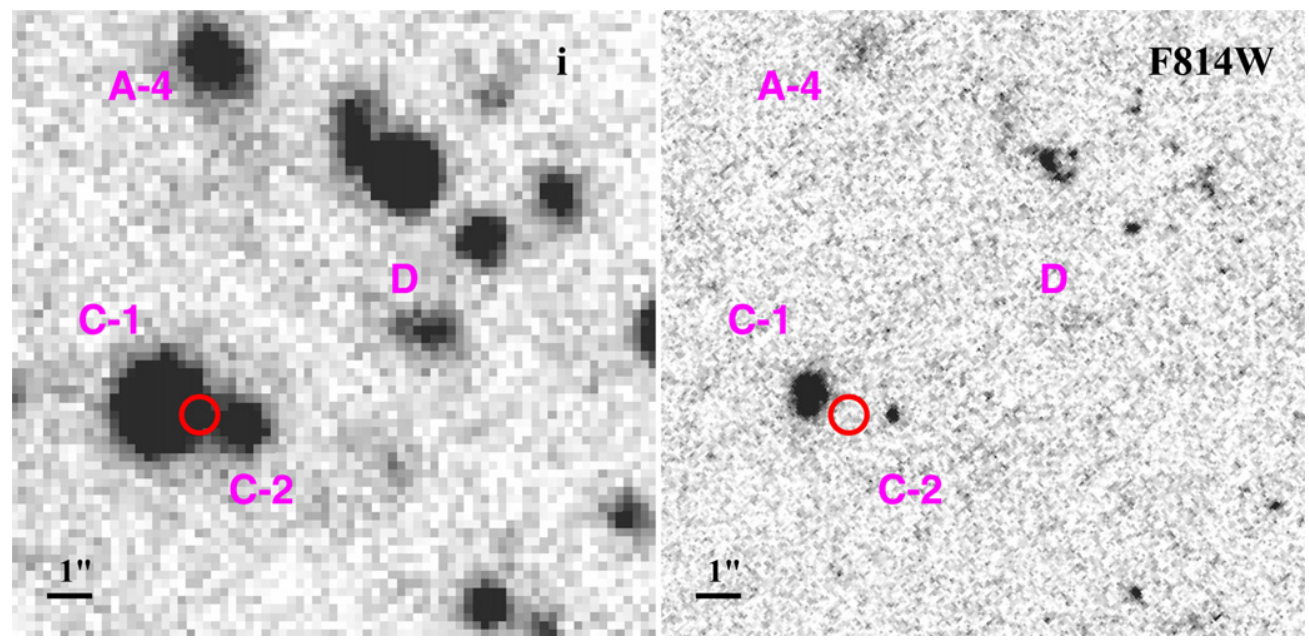

Figure 33. Similar to Figure 31, but for the $\mathbb{C}$ and $\mathbb{D}$ components of EGS19. The red circle indicates the position of $\mathrm{C}^{\prime}$, which is the geometric center of $\mathbb{C}-1$ and 2 (see Section 6.3.2).

(A color version of this figure is available in the online journal.)

to be used as the morphological templates, we refrained from doing TFIT of the IRAC image. Therefore, we only rely on the optical images from the CFHTLS Deep program to carry out the SED analysis. The photometry of the CFHTLS-Deep data was done in the same way as described in the previous sections.

The SED fitting results are summarized in Figure 34 for $\mathbb{A}$ and in Figure 35 for $\mathbb{B}, \mathbb{C}$, and $\mathbb{D}$. From the best-fit models and the $P(z)$ distributions, we concluded that $\mathbb{A}-1,2$, and 4 could be at the same redshift and associated. Taking the average, we adopted $z_{\mathrm{ph}}=1.06 \pm 0.07$ as their common redshift. For the decomposition purpose below, we took the geometric center of these three objects as the common center of the system, and denoted this new "object" as $\mathrm{A}^{\prime}$. Our assumption is that $\mathrm{A}^{\prime}$ is the part from the $\mathbb{A}$ clump that have significant FIR contribution, and that all other components in this clump can be ignored. Similarly, $\mathbb{B}-1,2$, and 3 could be at the same redshift of $z_{\mathrm{ph}}=2.72 \pm 0.07$ and associated, and the new "object" at their geometric center is denoted as $\mathrm{B}^{\prime}$. The situation for $\mathbb{C}-1$ and 2 is somewhat uncertain because their $z_{\text {ph }}$ differ significantly. However, since the $P(z)$ distribution for $\mathbb{C}-2$ has a wide, flat peak that contains the sharp $P(z)$ peak of $\mathbb{C}-1$, it is reasonable to assume that they are actually associated. Therefore we adopt the best-fit $z_{\mathrm{ph}}=1.08$ for $\mathbb{C}-1$ as the common redshift for this system, which is denoted as $\mathrm{C}^{\prime}$, and assign the position at the geometric center. This also suggests that $C^{\prime}$ could be with the same group as $\mathrm{A}^{\prime}$, however, it is not appropriate to combine the two for the decomposition as their separation is too large. Finally, we note that the single object $\mathbb{D}$ might be within the same group as $\mathrm{B}^{\prime}$, however, this is highly uncertain due to the lack of prominent peaks in its $P(z)$ distribution.

\subsubsection{Decomposition in Mid-to-far-IR}

The decomposition was done at the locations of $\mathrm{A}^{\prime}, \mathrm{B}^{\prime}, \mathrm{C}^{\prime}$, and $\mathbb{D}$. At $24 \mu \mathrm{m}$, the automatically iterative decomposition converged at all four positions, albeit with significant residuals, which indicate that the other components that we ignored are non-negligible in this band. The decomposition failed in $70 \mu \mathrm{m}$ due to the low $\mathrm{S} / \mathrm{N}$ of the data, therefore we had to ignore this band. The automatic decomposition failed in 100 and $160 \mu \mathrm{m}$, presumably due to the insufficient $\mathrm{S} / \mathrm{N}$ in these bands. The trail-and-error fit at $100 \mu \mathrm{m}$ resulted in $\mathrm{A}^{\prime}$ and $\mathrm{C}^{\prime}$ as the contributors, however, in $160 \mu \mathrm{m}$ it could only settle on $\mathrm{A}^{\prime}$ and $\mathbb{D}$.
In both cases notable residuals and/or over subtractions could be seen at the positions of the other objects that did not get fitted. Forcing the fit with other combinations of objects either resulted in worse residuals or crashed the program. Nevertheless, the results for $\mathrm{A}^{\prime}$ were repeatable, and therefore we believed that the decomposition was successful for $\mathrm{A}^{\prime}$ in these two bands. In $250 \mu \mathrm{m}$, the automatically iterative decomposition was successful for all the four "objects," which is show in Figure 36. $\mathrm{A}^{\prime}$ takes $\sim 51 \%$ of the total flux and hence is the major contributor. Therefore our further discussion will only include $\mathrm{A}^{\prime}$. Table 2 summarizes the flux densities of this major component.

\subsubsection{Total IR Emission and Stellar Populations}

The only X-ray source within the EGS19 area is right on $\mathbb{C}-1$ (positional offset of only 0 '. 3 ). It has full-band $0.5-10 \mathrm{keV}$ flux of $1.46_{-0.22}^{+0.24} \times 10^{-16} \mathrm{erg} \mathrm{s}^{-1} \mathrm{~cm}^{-2}$, which corresponds to a total Xray luminosity in rest-frame $1.1-21.8 \mathrm{keV}$ of $1.16 \times 10^{43} \mathrm{erg} \mathrm{s}^{-1}$, implying that $\mathbb{C}-1$ most likely has an AGN. The lack of X-ray detections at $\mathrm{A}^{\prime}$ suggests that the FIR emission of this object is most likely of stellar origin, as the sensitivity limit would imply an upper limit of $1.8 \times 10^{41} \mathrm{erg} \mathrm{s}^{-1}$ in rest-frame $1-4 \mathrm{keV}$ at $z=1.06$.

Figure 37 summarizes the analysis of the mid-to-far-IR SED of $\mathrm{A}^{\prime}$. The SK07 models provided a good fit, re-assuring that our decomposition results for $\mathrm{A}^{\prime}$ is reasonable. We obtained $L_{\mathrm{IR}}=1.0 \times 10^{12} M_{\odot}$, which means that $\mathrm{A}^{\prime}$ is an ULIRG. The analytic fit resulted in $T_{d}^{\mathrm{fit}}=34.0 \mathrm{~K}$ and $M_{d}=4.0 \times 10^{8} M_{\odot}$. This implies $M_{\text {gas }}=5.6 \times 10^{10} M_{\odot}$.

The dominant members of $\mathrm{A}^{\prime}$ are $\mathbb{A}-1$ and $\mathbb{A}-4$, which have extremely similar stellar populations. They both have moderate stellar masses (4.1 and $2.9 \times 10^{10} M_{\odot}$, respectively), old ages (2.0 and $1.7 \mathrm{Gyr}$, respectively), and moderately prolonged SFH ( $\tau=0.7$ and $0.4 \mathrm{Gyr}$, respectively). Combining $\mathbb{A}-1$ and $\mathbb{A}-4$, we got $L_{\mathrm{IR}}^{\text {ext }}=8.1 \times 10^{10} L_{\odot}, L_{\mathrm{IR}}^{\text {blk }}=9.2 \times 10^{11} L_{\odot}$, and $\mathrm{SFR}_{\mathrm{IR}}^{\text {blk }}=92 M_{\odot} \mathrm{yr}^{-1}$. Their combined $\mathrm{SFR}_{\text {fit }}=8 M_{\odot} \mathrm{yr}^{-1}$, and thus $\mathrm{SFR}_{\mathrm{tot}}=100 M_{\odot} \mathrm{yr}^{-1}$. Using their stellar masses, we can get SSFR $=1.4 \mathrm{Gyr}^{-1}, T_{\mathrm{db}}^{\text {tot }}=700 \mathrm{Myr}$, and $T_{\mathrm{db}}^{\mathrm{blk}}=760 \mathrm{Myr}$. The total amount of gas inferred above could fuel this ULIRG for the next $560 \mathrm{Myr}$ or its dust-blocked region for the next $608 \mathrm{Myr}$ to add a further $\sim 80 \%$ of its existing stellar masses. 

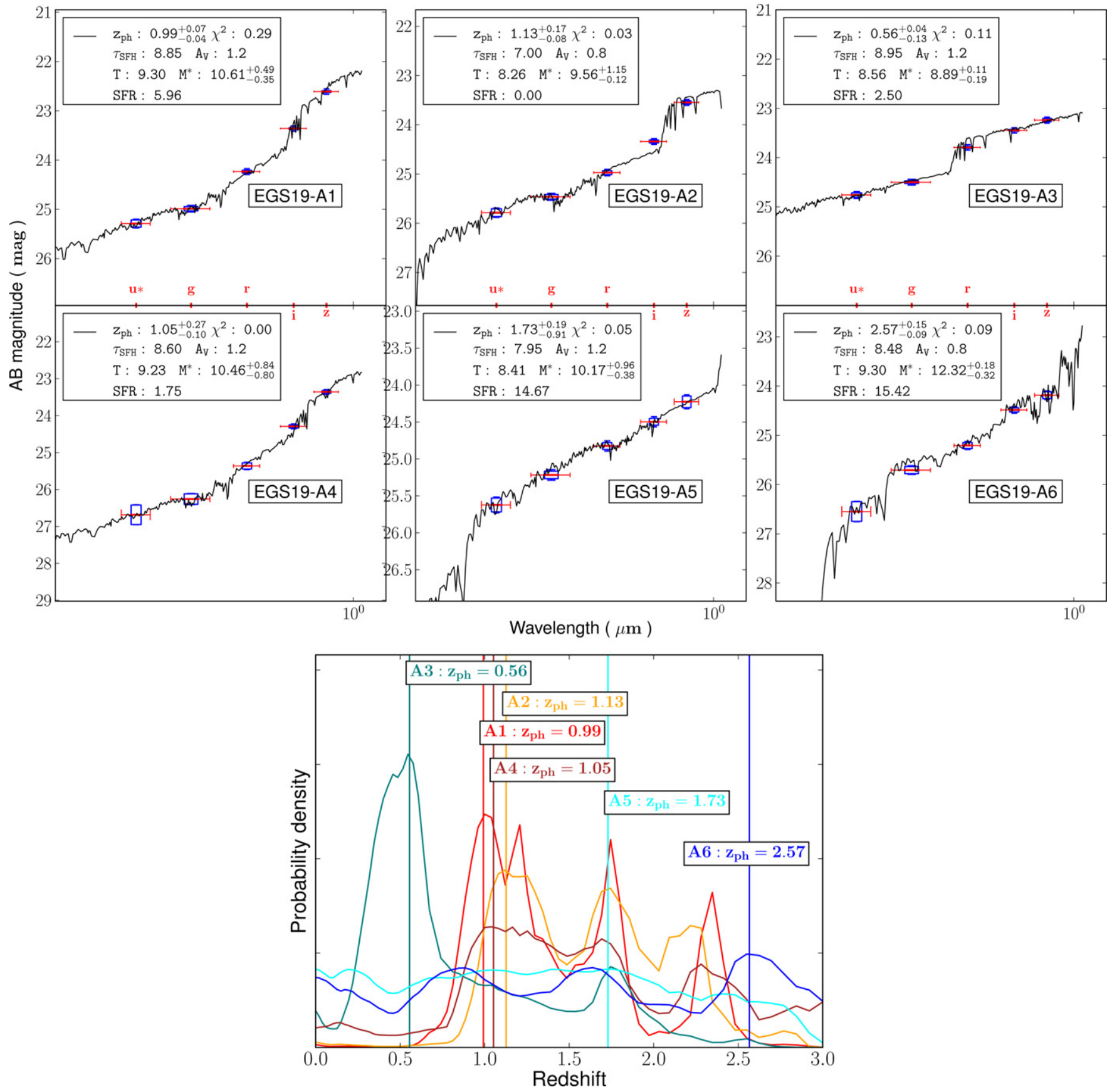

Figure 34. Optical SED fitting for the subcomponents of the EGS19- $\mathbb{A}$ clump. Legends are the same as in Figure 4 . $\mathbb{A}-1,2$, and 4 could be at the same redshift of $z_{\mathrm{ph}}=1.06$ and associated.

(A color version of this figure is available in the online journal.)

There are two radio sources in this region (Ivison et al. 2010). One of them coincides with $\mathbb{A}-1$, and has $S_{1.4 \mathrm{GHz}}=$ $0.107 \pm 0.012 \mathrm{mJy}$. The other one is on top of $\mathbb{B}-1$, and has $S_{1.4 \mathrm{GHz}}=0.119 \pm 0.012 \mathrm{mJy}$. Here we only discuss the FIRradio relation for the former. We obtained $q_{\mathrm{IR}}=2.27$, which is lower but still consistent with the mean of $2.40 \pm 0.24$ in Ivison et al. (2010).

\section{DISCUSSION}

Strictly speaking, our sample of SDSS-invisible, bright Herschel sources is not flux-limited and could suffer from various types of incompleteness. Nonetheless, our study can serve as a guide to future investigations of similar objects at larger, more complete scales.

\subsection{Decomposition of Bright Herschel Sources}

Our decomposition approach provides a promising solution to maximize the returns of the large volume of precious Herschel data. Our technique is based on the position priors from optical or near-IR images, and this is different from those using the position priors from the MIPS $24 \mu \mathrm{m}$ images, which suffer from the blending problem themselves. While it probably cannot decompose all the contributors to a given Herschel source, our method is capable of at least identifying its major components 

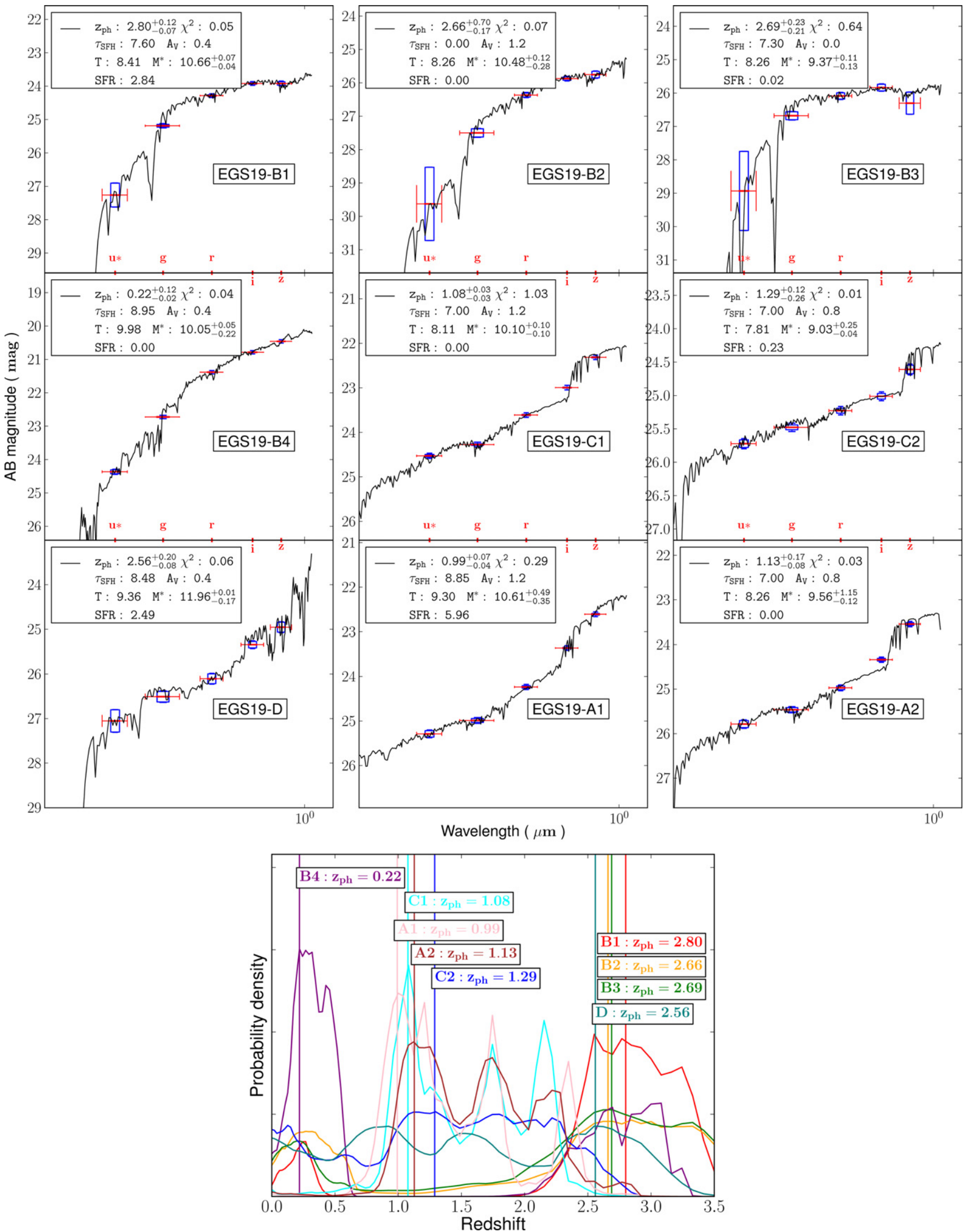

Figure 35. Similar to Figure 34, but for the EGS19- $\mathbb{B}$, $\mathbb{C}$, and $\mathbb{D}$ clumps. Legends are the same as in Figure 4 . Based on their $P(z)$ distribution, $\mathbb{B}-1,2$, and 3 could be at the same redshift of $z_{\mathrm{ph}}=2.72$. Similarly, $\mathbb{C}-1$ and 2 could also be at the same redshift of $z_{\mathrm{ph}}=1.08$, and might even be within the same $\mathbb{A}$ group. $\mathbb{D}$ could be within the same $\mathbb{B}$ group.

(A color version of this figure is available in the online journal.) 

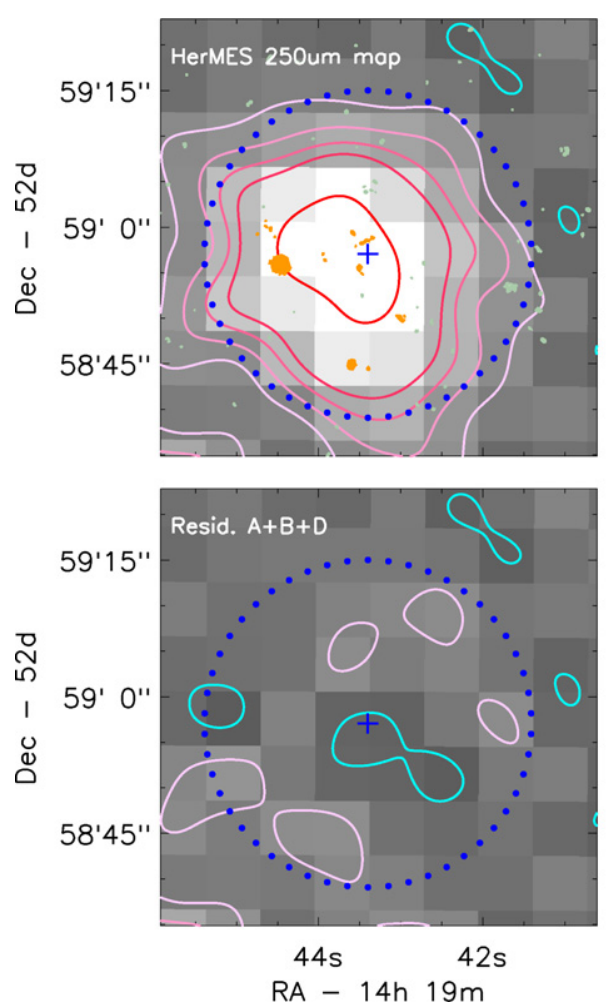
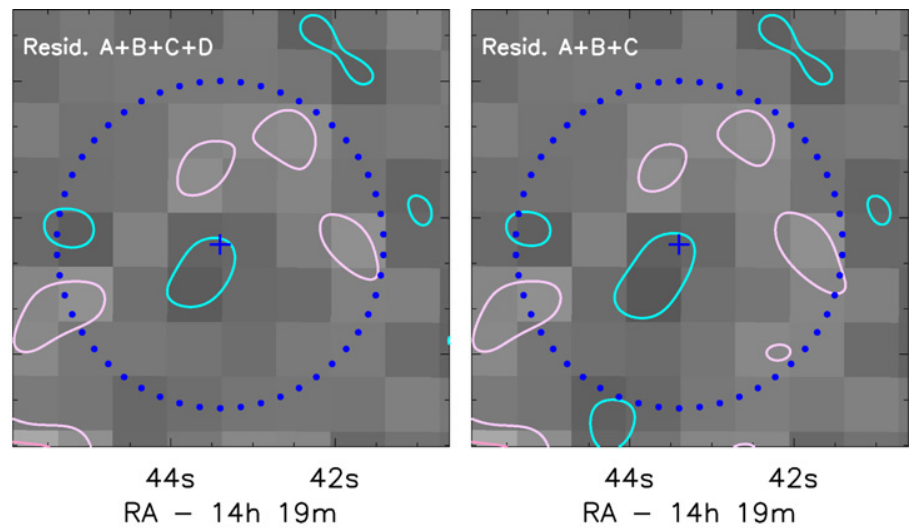

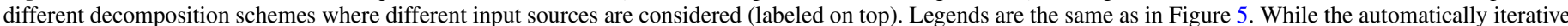

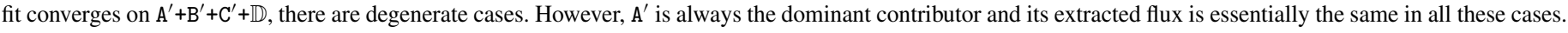
(A color version of this figure is available in the online journal.)
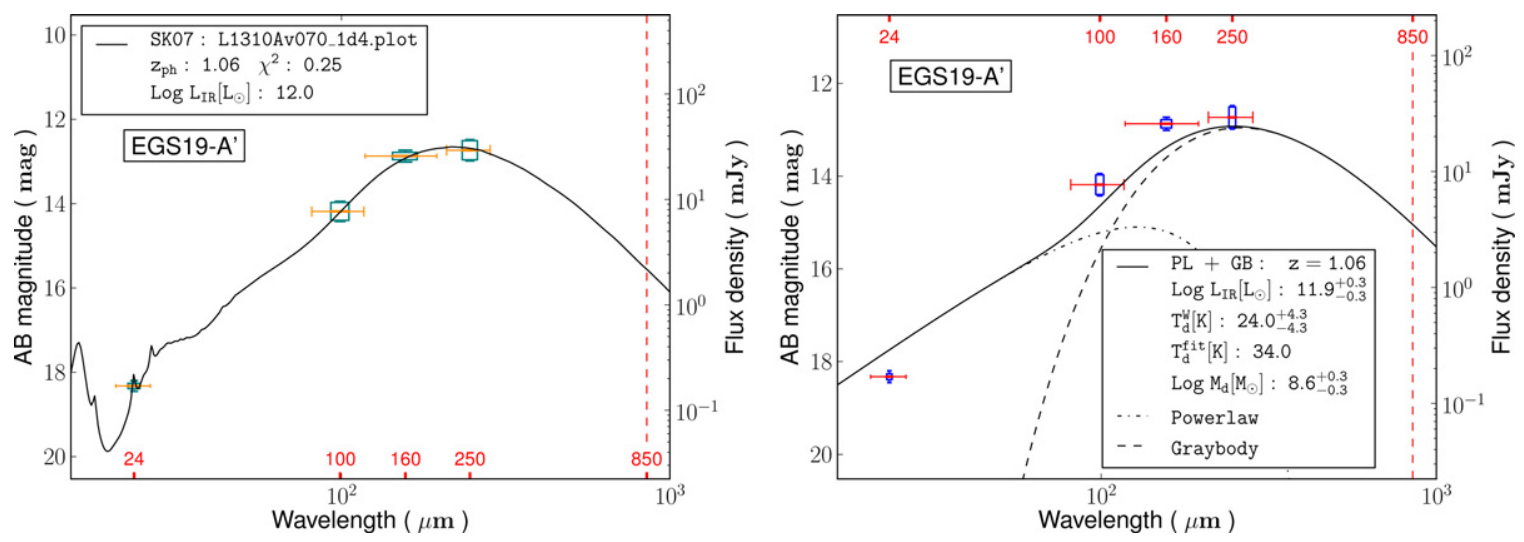

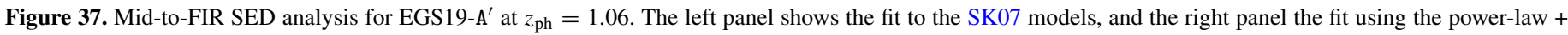
graybody models. Legends are the same as in Figure 6.

(A color version of this figure is available in the online journal.)

and extracting their fluxes. This method should work even when only using medium-deep optical images for position priors. For example, in the case of UDS01, our results would largely remain the same should we use the priors from the CFHTLS-Wide data instead of the CANDELS WFC3 IR data, because the input list would essentially be the same.

Two interesting conclusions can be drawn from our decomposition. First, as GOODSN63 shows, the brightest $24 \mu \mathrm{m}$ source in the Herschel beam could have no contribution in the FIR, and therefore the use of $24 \mu \mathrm{m}$ data should be exercised with caution and not to limit to only the brightest $24 \mu \mathrm{m}$ sources for position priors. Second, a bright Herschel source usually is the collective result of multiple contributors. While the seven sources investigated here all have $S_{250}>55 \mathrm{mJy}$, and hence are $\sim 9.5 \times$ above the nominal confusion limit of $5.8 \mathrm{mJy}^{\text {beam }}{ }^{-1}$ in HerMES at the SPIRE $250 \mu \mathrm{m}$ (Nguyen et al. 2010), it is not guaranteed that they do not suffer from the blending problem. In fact, only two (GOODS63 and UDS04) out these seven sources can be safely treated as having only one contributor. In two other cases (UDS01 and EGS14), the source is made of two major, distinct components that are likely at the same redshifts and associated. In the other three cases (GOODSN06, EGS07, and EGS19), while there is always a primary component contributing most of the FIR flux, the other contributors, which are not physically associated with the primary component, are still non-negligible.

Both conclusions above actually echo the results of earlier studies of SMGs using the SMA (Younger et al. 2009; Wang 
Table 3

Physical Properties of Revealed ULIRGs

\begin{tabular}{|c|c|c|c|c|c|c|c|c|c|c|c|c|c|c|c|}
\hline ID & $z$ & $\begin{array}{c}L_{\mathrm{IR}} \\
\left(10^{12} L_{\odot}\right)\end{array}$ & $\begin{array}{c}L_{\mathrm{IR}}^{\mathrm{blk}} \\
\left(10^{12} L_{\odot}\right)\end{array}$ & $\begin{array}{c}S_{850} \\
(\mathrm{mJy})\end{array}$ & $\begin{array}{c}\mathrm{SFR}_{\mathrm{IR}}^{\mathrm{blk}} \\
\left(M_{\odot} / \mathrm{yr}\right)\end{array}$ & $\begin{array}{l}T_{d}^{\mathrm{fit}} \\
(\mathrm{K})\end{array}$ & $\begin{array}{c}M_{d} \\
\left(10^{8} M_{\odot}\right)\end{array}$ & $\begin{array}{c}M_{\text {gas }} \\
\left(10^{10} M_{\odot}\right)\end{array}$ & $\begin{array}{c}M^{*} \\
\left(10^{10} M_{\odot}\right)\end{array}$ & $\begin{array}{c}\mathrm{SFR}_{\mathrm{fit}} \\
\left(M_{\odot} / \mathrm{yr}\right)\end{array}$ & $\begin{array}{c}\tau \\
(\mathrm{Gyr})\end{array}$ & $\begin{array}{c}T \\
(\mathrm{Gyr})\end{array}$ & $\begin{array}{l}\mathrm{SSFR}_{\text {tot }} \\
\left(\mathrm{Gyr}^{-1}\right)\end{array}$ & $\begin{array}{c}T_{\mathrm{db}}^{\mathrm{tot}} \\
(\mathrm{Gyr})\end{array}$ & $\begin{array}{c}T_{\mathrm{db}}^{\mathrm{blk}} \\
(\mathrm{Gyr}) \\
\end{array}$ \\
\hline GOODSN06-A & 1.225 & 4.0 & 1.7 & 2.9 & 170 & 48.7 & 4.0 & 5.6 & 3.2 & 236 & 0.02 & 0.045 & 12.7 & 0.079 & 0.188 \\
\hline GOODSN63-A ${ }^{\mathrm{a}}$ & 2.28 & 7.9 & 7.8 & 9.8 & 781 & 39.5 & 39.8 & 55.7 & 46.8 & 11 & 0 & 2.0 & 1.7 & 0.593 & 0.602 \\
\hline UDS01-B & 1.042 & 1.0 & 0.48 & 1.5 & 48 & $\ldots$ & $\ldots$ & $\ldots$ & 4.6 & 0 & 0 & 0.13 & 1.0 & 1.0 & 1.0 \\
\hline UDS01-D & 1.042 & 1.0 & 0.62 & 1.5 & 62 & $\ldots$ & $\ldots$ & $\ldots$ & 7.9 & 39 & 1.0 & 1.4 & 1.2 & 0.882 & 1.3 \\
\hline$(\mathrm{UDS} 01-\mathrm{BD})^{\mathrm{b}}$ & 1.042 & 2.0 & $\ldots$ & $\ldots$ & $\ldots$ & 39.2 & 5.0 & 7.0 & 12.5 & $\ldots$ & $\ldots$ & $\ldots$ & $\ldots$ & $\ldots$ & $\ldots$ \\
\hline UDS04-A & 1.267 & 5.0 & 2.5 & 3.7 & 255 & 38.1 & 10.0 & 14.0 & 10.2 & 208 & 0.08 & 0.18 & 4.6 & 0.216 & 0.392 \\
\hline EGS07-1 ${ }^{\mathrm{c}}$ & 1.497 & 4.0 & $\ldots$ & 5.4 & $\ldots$ & 42.1 & 12.6 & 17.6 & 6.9 & 137 & 7.0 & 0.72 & $\ldots$ & $\ldots$ & . \\
\hline EGS14-5 & 1.15 & 1.3 & 0.83 & 2.1 & 83 & 36.9 & 4.0 & 5.6 & 30.2 & 45 & 4.0 & 5.5 & 0.4 & 2.3 & 3.6 \\
\hline EGS14-9 & 1.15 & 1.0 & 0 & 2.1 & 0 & 37.2 & 3.2 & 4.4 & 5.0 & 72 & 0.04 & 0.1 & 1.4 & 0.694 & $\ldots$ \\
\hline EGS19-A' & 1.06 & 1.0 & 0.92 & 2.1 & 92 & 34.0 & 4.0 & 5.6 & 7.0 & 8 & 0.55 & 1.8 & 1.4 & 0.560 & 0.608 \\
\hline
\end{tabular}

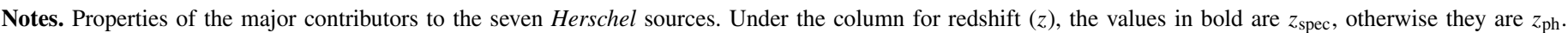

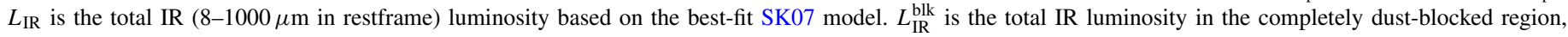

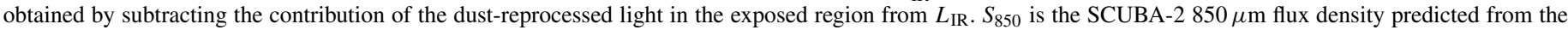

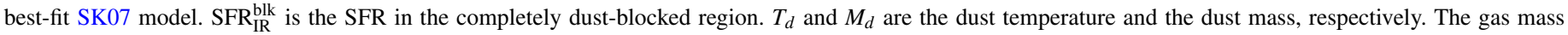

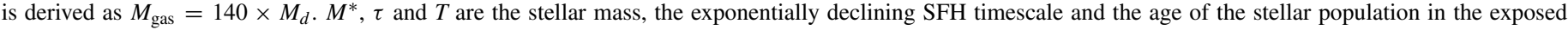

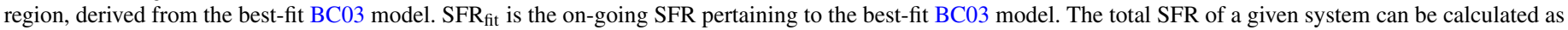

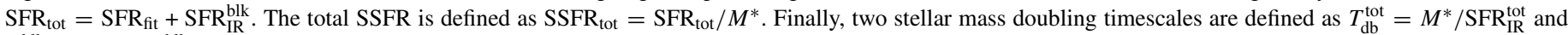
$T_{\mathrm{db}}^{\mathrm{blk}}=M^{*} / \mathrm{SFR}_{\mathrm{IR}}^{\mathrm{blk}}$.

a For the stellar population of this object, the listed age $(T)$ and the characteristic SFH timescale $(\tau)$ are for the "red" component in its SED. See Figure 9.

$\mathrm{b}$ This is not an independent object but for the case where the two components (UDS01-B and D) are combined.

c This object very likely has an AGN, which could be responsible for the IR emission.

et al. 2011; Barger et al. 2012) and the more recent ones using the ALMA (Hodge et al. 2013; Karim et al. 2013). Our second point, namely, that in most cases bright Herschel sources are made of distinct components, will impact the interpretation of the FIR source counts and the construction of the FIR luminosity functions, especially at the bright end. This is now expected from the theoretical side. Niemi et al. (2012) suggest that source blending could be an important cause of the inconsistency between their semi-analytic model predictions and the actual observations of the bright Herschel source number counts. Recently, using a cosmological numerical simulation, Hayward et al. (2013) predicted that spatially and physically unassociated galaxies contribute significantly to the SMG population. Therefore, bright Herschel sources and their multiplicities warrant further investigations. As compared to addressing this problem through using submillimeter interferometry with the SMA or the ALMA, our approach offers a much less expensive alternative, albeit at the price that we are only able to reliably extract the major component(s) of a given Herschel source. However, as neither the SMA nor the ALMA is a survey machine, our method has its value in that, in principle, it can deal with a large number of sources. In addition, it is applicable in the Herschel fields where there are no MIPS $24 \mu \mathrm{m}$ data as "ladders" but medium-deep optical imaging data are available or can be acquired. While in this work we still used the $24 \mu \mathrm{m}$ image to narrow down the input list for the fit, it is possible to get rid of this intermediate step once we automate the current trial-and-error approach (Z. Ma et al., in preparation). The Appendix further demonstrates this point. Of course, relying on optical images for priors does have its disadvantage in that a major contributor to the FIR source, if extremely dusty, could still be missing from a medium-deep optical survey. In this case, the residual image after the decomposition will be able to reveal such a source. In fact, in our trial-and-error approach for a number of sources, the iteration was driven by the residual left at the locations of the suspected contributors.

\subsection{ULIRG Diagnostics}

Our decomposition directly identified the optical-to-near-IR counterparts of the major components of the FIR sources. This allows us to investigate the nature of the FIR emission and the underlying stellar populations. We have found the following.

First of all, the major contributors to our FIR sources are all ULIRGs at $z>1$. Although this is not surprising given their high FIR flux densities (suggesting high IR luminosities) and faintness in optical (suggesting being at high redshifts), our analysis provides solid evidence that this is indeed the case. While one of these sources (EGS07) could have an embedded AGN, all others are mainly powered by intense star formation heavily obscured by dust.

The exquisite morphological details in the rest-frame optical from the HST data show that these ULIRGs all have complicated structures indicating either a merger or local violent instability. This is generally consistent with the result of Kartaltepe et al. (2012), who find that the majority of the PACS-selected ULIRGs at $z \sim 2$ in the GOODS-South field are mergers and irregular galaxies. While a quantitative morphological modeling is beyond the scope of this work, most of the counterparts to the ULIRG in our sample are not likely to be explained by a single disk galaxy. This is in contrast to the recent study of Targett et al. (2013), who find that SMGs, being ULIRGs at $z \approx 2$, are mostly disk galaxies. This contrast probably should not yet be viewed as a contradiction for two reasons. First, our objects are mostly at $z \approx 1$. Second, based on the predicted $S_{850}$ (see Table 3 ) of our objects, most of them probably are not SMGs based on the conventional SMG selection criterion ( $S_{850} \gtrsim 3-5 \mathrm{mJy}$; see also Khan et al. 2009). Nonetheless, one of our objects, GOODSN63-A, is an SMG at $z_{\mathrm{ph}}=2.28$ and apparently cannot be a disk galaxy. Clearly, the morphologies of high- $z$ ULIRGs merit further investigation.

The assertion that our sources are all ULIRGs is based on total IR luminosities derived from the direct measurements in 

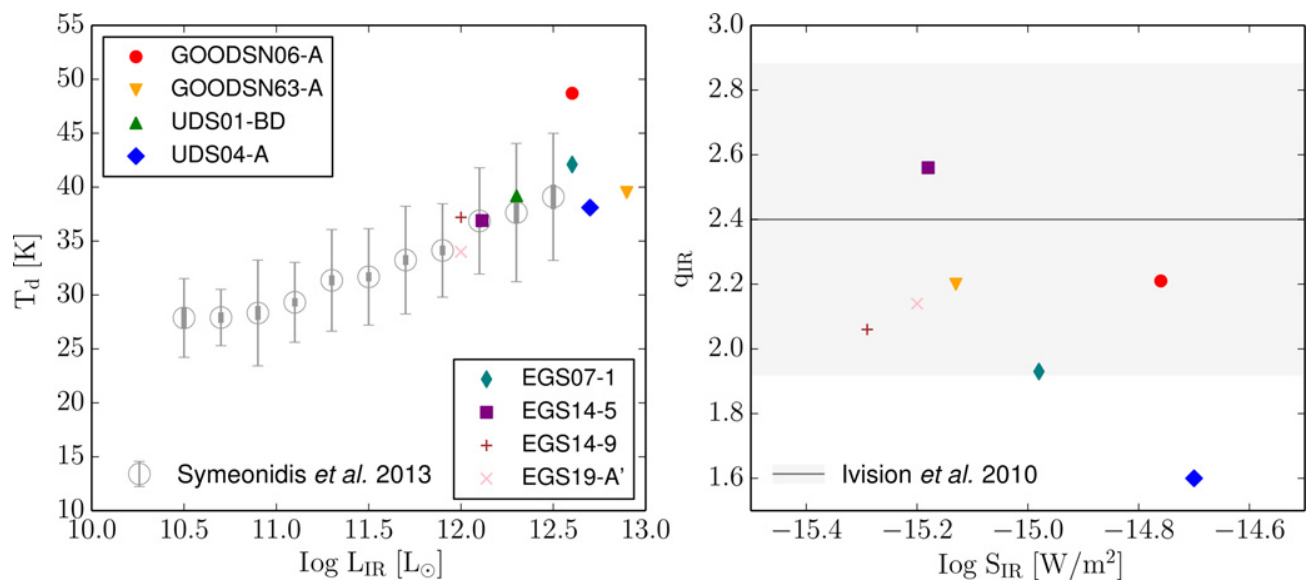

Figure 38. Left: dust temperature of ULIRG as a function of total IR luminosity. The circles with error bars are the mean values from Symeonidis et al. (2013) whose sample is at $0.1<z<2$, while the results from our sample (as labeled) are plotted as symbols of various colors. Right: comparison of $q_{\mathrm{IR}}$ from our sample, shown as the open squares, to the result of Ivison et al. (2010). The solid horizontal line indicates their mean value $\left(q_{\mathrm{IR}}=2.4\right)$ and the gray area represents their $\pm 2 \sigma_{q}= \pm 0.48$. (A color version of this figure is available in the online journal.)

the FIR bands that have been properly treated for the effect of blending. They are more reliable than the extrapolation from the mid-IR (e.g., using only the MIPS $24 \mu \mathrm{m}$ in the pre-Herschel era). The multiple Herschel FIR bands sample the peak of dust emission, and thus are more sensitive in selecting ULIRGs than using a single submillimeter band. In fact, we find that only two ULIRGs in our sample (GOODSN63-A and EGS07-1) would satisfy the nominal SMG selection criterion of $S_{850}>5 \mathrm{mJy}$. The Herschel bands are also sensitive to a wide temperature range and are not biased against ULIRGs of high temperatures as the traditional SMG selection at $850 \mu \mathrm{m}$ is (e.g., Chapman et al. 2010; Casey et al. 2012). Indeed, our sample includes one ULIRG whose dust temperature is higher than those of normal SMGs (GOODSN06-A with $T_{d}^{\text {fit }}=48.7 \mathrm{~K}$ ). As shown in Figure 38 (left panel), our small sample already shows a trend that dust temperature increases with respect to increasing IR luminosity, and this is consistent with the results in the recent literature (e.g., Symeonidis et al. 2013; Swinbank et al. 2014; Magnelli et al. 2014). A much larger sample in the future, constructed following the decomposition process described in this work, will be able to enhance this trend (particularly at the bright end) and determine whether the wide dispersion currently seen is intrinsic or is due to the contamination to the FIR fluxes by blending.

We have also investigated the FIR-radio relation of our sources. We find that some of our sources follow the relation very well and yet some deviate from it significantly. Figure 38 (right panel) compares the $q_{\mathrm{IR}}$ values from our sample, which have the mean of $2.22 \pm 0.28$, to the mean of $2.40 \pm 0.24$ of Ivison et al. (2010). It seems that our values are systematically lower than theirs, however, our sample is too small for us to make any assertion. Nevertheless, we argue that using our method of decomposition will result in the most reliable measurement of $S_{\mathrm{IR}}$ and hence reduce the random measurement error in the dispersion, and that future studies using larger sample will be able to test the FIR-radio relation for $z>1$ ULIRG in better details.

It is interesting to relate our ULIRGs to the so-called "main sequence of star formation" on the SFR (or SSFR) versus stellar mass plane (e.g., Noeske et al. 2007; Elbaz et al. 2007, 2011; Daddi et al. 2007; Wuyts et al. 2011; Rodighiero et al. 2011; Leitner 2012). This is shown in Figure 39 in terms of SFR (left) and SSFR (right). In the left panel, the solid line represents the main sequence of Elbaz et al. (2007) at $z \approx 1$ and the dashed line $4 \times$ above it follows Rodighiero et al. (2011) to indicate the boundary above which starbursts locate $(\sim 2 \sigma$ above the main sequence). In the right panel, the solid line is from the empirical fit to the main sequence as a function of redshifts based on Leitner (2012), and is fixed at the median redshift $(z=1.2)$ of our sample. Somewhat surprisingly, some of our objects are quite close or below this boundary and would be difficult to qualify as starbursts in this convention. As it turns out, the existing stellar populations of these objects tend to have an old age ( $T>1$ Gyr). In particular, GOODSN63-A and EGS14-5, which are the closest to the main sequence in SSFR versus $M^{*}$, have the oldest ages.

The closest analogs to our objects are SMGs selected at $850 \mu \mathrm{m}$, which are mostly ULIRGs at $z \approx 1-3$. The stellar population studies of SMGs (Borys et al. 2005; Dye et al. 2008; Michałowski et al. 2010, 2012; Hainline et al. 2011; Targett et al. 2013) have come to the conclusion that they have very high stellar masses, with the quoted median ranging from $7 \times 10^{10} M_{\odot}$ to $2 \times 10^{11} M_{\odot}$. The recent numerical simulation of Hayward et al. (2011) shows that SMGs should have a minimum of $M^{*} \gtrsim 6 \times 10^{10} M_{\odot}$ and that typical masses should be higher. Michałowski et al. (2012) and Targett et al. (2013) have found mean values of $\left\langle M^{*}\right\rangle=(2.8 \pm 0.5) \times 10^{11}$ and $(2.2 \pm 0.2) \times 10^{11} M_{\odot}$, respectively. The mean of our sample is $\left\langle M^{*}\right\rangle=(1.4 \pm 1.5) \times 10^{11} M_{\odot}$, where the large dispersion reflects the fact that our estimates range from as low as $2.5 \times 10^{10} M_{\odot}$ to as high as $4.7 \times 10^{11} M_{\odot}$. In fact, six out of the nine objects are below $10^{11} M_{\odot}$ (see Table 2). While the differences in the assumed SFH could affect the stellar mass estimates systematically (for example, Michałowski et al. (2012) conclude that the estimates using their preferred twocomponent models are $2-3 \times$ higher than those using singlecomponent models), our large dispersion cannot be attributed to this reason. We believe that this is due to the fact that most of our objects are not SMGs. In other words, ULIRGs selected by Herschel bands are more heterogeneous in their existing stellar populations than SMGs. This probably is understandable given that starbursting galaxies at high redshifts are found over a wide range of stellar masses (e.g., Rodighiero et al. 2011). However, a starburst (defined as being an object far away from the "main sequence") is not necessarily a ULIRG, and therefore the spread in ULIRG stellar masses should not be taken for granted. It thus 

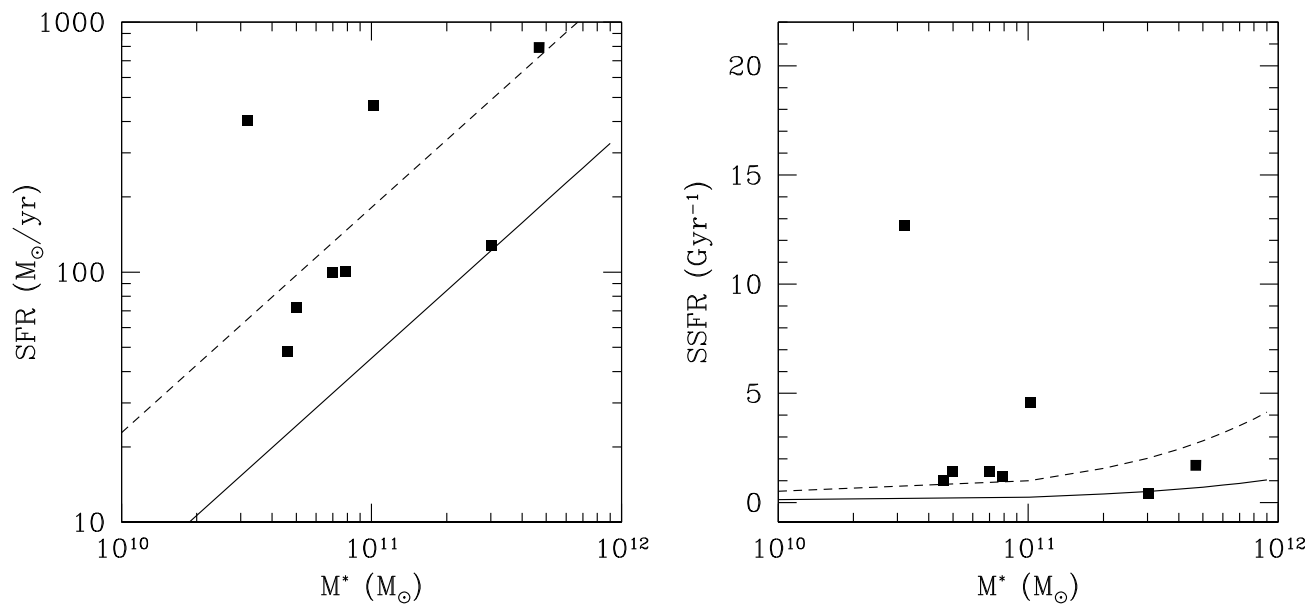

Figure 39. Locations of the ULIRGs in our sample with respect to the "star formation main sequence" on the SFR vs. $M^{*}$ (left) and the SSFR vs. $M^{*}$ (right) planes.

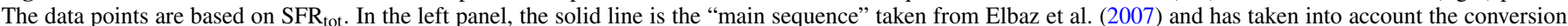
from the Salpeter IMF to the Chabrier IMF. The dashed line follows that of Rodighiero et al. (2011) and is $4 \times$ above the main sequence. In the right panel, the solid curve is the main-sequence power-law fit taken from Leitner (2012; using the data from Karim et al. 2011) and fixed at the median redshift $(z=1.2)$ of our sample. The dashed curve is $4 \times$ above the main sequence. The objects above the dashed line/curve can be taken as "starbursts" in this context. Some of our ULIRGs are actually quite close to the main sequence.

will be important to further investigate this question with a much increased, properly deblended sample.

While the stellar mass obtained through SED fitting is robust, it is known that the age inferred from the same process can be more uncertain (see, e.g., Wuyts et al. 2011 for a recent reference). Presenting a comprehensive examination to address the age estimate issue is beyond the scope of this paper. Nevertheless, we argue that the age estimate in our case could still be useful. Dust extinction and metallicity are the two most severe sources of uncertainty. In the SED fitting for the existing (exposed) stellar populations, we allowed $A_{V}$ to vary from 0 to 4 mag, and assumed solar metallicity. As our objects are rather dusty, there is a chance that we might still underestimate the extinction. However, these objects could have lower metallicity because they are at $z>1$. Generally speaking, a higher extinction would tend to result in a younger age in the fit because it would make the templates redder, and a lower metallicity would tend to result in a older age because it would make the templates bluer. Therefore, the two possible biases in our treatment tend to cancel out. For this reason, we believe that the ages that we derived still provide useful hints to understanding the progenitor of these ULIRGs.

The derived ages of our objects have a wide spread, ranging from as young as $\sim 20 \mathrm{Myr}$ to as old as almost the age of the universe at the observed redshift and do not have any obvious trend with respect to the existing stellar mass. This implies that the progenitor of a ULIRG could be formed at any redshift, and that the ULIRG phase can be turned on at any point during its lifetime. Furthermore, it can be turned on at any mass range within $10^{10-11} M_{\odot}$. This is in contrast to the SMG population, where the observed ULIRG phase is believed to only happen at the late stage of their stellar mass assembly and probably will not add much to the existing mass (see, e.g., Michałowski et al. 2010).

In light of this, we propose new diagnostics of ULIRGs, which are shown as 3D plots in Figure 39 for six ULIRGs from Table 3. The ratio $M_{\text {gas }} / M^{*}$ is a measure of the gas content in the ULIRG region with respect to the existing stellar mass in the exposed region. If $M_{\mathrm{gas}} / M^{*}>1$, in principle the ULIRG will be capable of doubling the mass. The ratio $T_{\mathrm{db}} / T$ is a measure of how quickly the ULIRG can double the mass as compared to the age of the existing stellar population..$^{18}$ The age $T$ is a measure of how closely the current ULIRG is related to the past SFH, i.e., it can be understood as at what stage the ULIRG happens since the birth of the galaxy. Finally, $M^{*}$, together with $T$, indicates how mature the existing stellar population is.

It is intriguing to see how these six ULIRGs spread in the $M_{\mathrm{gas}} / M^{*}-T_{\mathrm{db}}^{\mathrm{blk}} / T-T-M^{*}$ space. Two of them, namely, GOODSN06-A and UDS04-A, are the most gas rich and the most efficient in their mass assembly. As their hosts are all quite young, these ULIRGs are likely very close to, or even an extension of the past episode of active star formation in the hosts. The next in line is GOODSN63-A, which is capable of doubling its existing mass in a period even shorter than the age of the host. Considering that it has a very high stellar mass already (the highest among all of the six), this is rather extreme. However, its current ULIRG phase should have little to do with the bulk of the assembly of the existing mass given the old age of the host. The gas reservoirs of EGS14-9 and EGS19- $\mathrm{A}^{\prime}$ are somewhat insufficient for them to double their existing masses, and hence their current ULIRG phase is less important in terms of stellar mass assembly. In this regard, the current ULIRG phase of EGS14-5 is the least important, as it does not have a sufficient amount of gas to get close to double the existing mass. However, it is also very unusual that a very high mass, extremely old galaxy like EGS14-5 still has not yet shut off its star formation processes at $z \approx 1$.

All this suggests that the high- $z$ ULIRGs make a diverse population. The diagnostics such as shown in Figure 40 have the potential of revealing the role of ULIRGs in assembling high-mass galaxies. For this purpose, a much larger sample will be necessary.

\section{SUMMARY}

We studied a sample of seven very bright Herschel sources $\left(S_{250}>55 \mathrm{mJy}\right)$ from the HerMES program that are not visible

\footnotetext{
18 Numerically, $T_{\mathrm{db}} / T$ is equivalent to $1 / b$, where $b$ is the "birthrate" parameter defined as $b=\mathrm{SFR} /\langle\mathrm{SFR}\rangle$ and $\langle\mathrm{SFR}\rangle=M^{*} / T$ (Scalo 1986).
} 


\begin{tabular}{|llll|}
\hline$\bullet$ & GOODSN06-A & $\diamond$ & EGS14-05 \\
¿ & GOODSN63-A & $\bullet$ & EGS19-A' \\
UDS04 & & \\
\hline
\end{tabular}

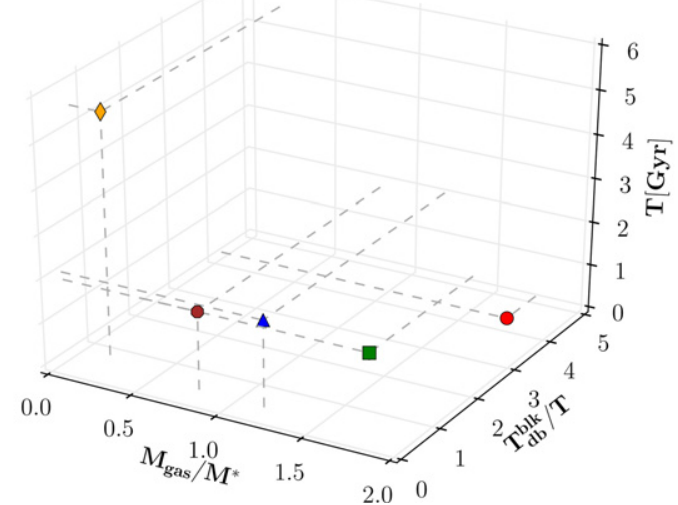

\begin{tabular}{|llll|}
\hline$\bullet$ & GOODSN06-A & $\diamond$ & EGS14-05 \\
\} $&{\text { GOODSN63-A }} &{\bullet} &{\text { EGS19-A' }} \\
{\text { UDS04 }} &{ } &{ } &{ } \\
{\hline}$
\end{tabular}

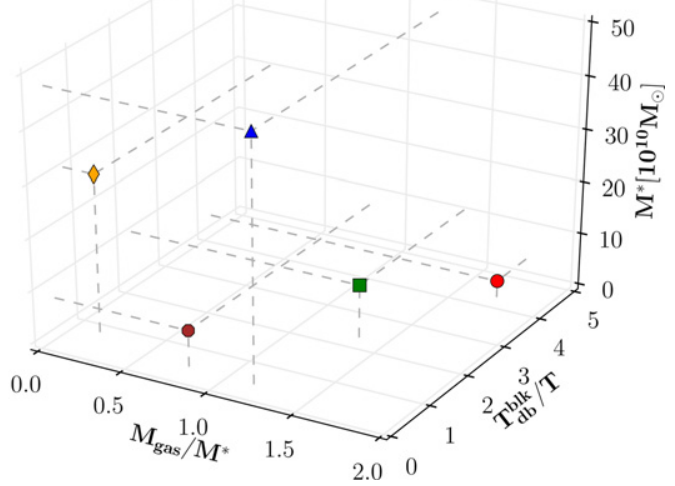

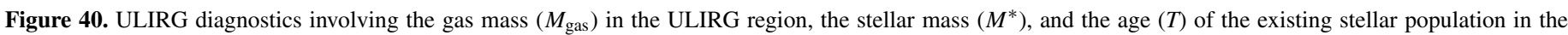
exposed region, and the stellar mass doubling time $T_{\mathrm{db}}$ due to ULIRG assuming a constant SFR $\mathrm{IR}_{\mathrm{I}}$ into the future.

(A color version of this figure is available in the online journal.)

in the SDSS. Such sources have a surface density of roughly $10 \mathrm{deg}^{-2}$, and comprise a non-negligible fraction of the brightest FIR sources on the sky. In order to understand their nature, we selected these seven sources in particular because they are in the CANDELS fields where a rich set of multi-wavelength data sets are available for our study.

Due to the large beam sizes of the Herschel instruments, the deep optical and near-IR images in these fields readily reveal many possible counterparts within the footprints of these FIR sources. To combat this problem, we took a new approach to decompose the heavily blended potential counterparts, using their centroids at the high-resolution near-IR or optical images as the position priors. Such an elaborated counterpart identification is superior to the simple treatment of using a mid-IR proxy such as the MIPS $24 \mu \mathrm{m}$ image and claiming the brightest mid-IR source within the FIR beam as the counterpart. In fact, we show that in at least one case (GOODSN63) the brightest $24 \mu \mathrm{m}$ source within the $250 \mu \mathrm{m}$ beam actually does not contribute to the FIR emission, which agrees with the SMA interferometry. Our method is also an improvement over using the mid-IR data for position priors, as such images are often already confused in the first place. In this regard, our approach has the advantage of unambiguously identifying the counterparts and extracting their fluxes at the same time. While in this work we still use the MIPS $24 \mu \mathrm{m}$ data to narrow down the number of input objects to the fitting procedure, it is possible to eliminate this step when we fully automate the entire process in the near future (see the Appendix). Once implemented, the automatic routine will have a wide application in the Herschel fields where the $24 \mu \mathrm{m}$ data are not available.

Our result shows that in most cases multiple objects contribute to the ostensibly single FIR source. While some of them are multiples at the same redshifts, others are superposition by chance and are physically unrelated. If left untreated, the contamination to the FIR flux due to the latter case could be as high as $40 \%$. In either case, the decomposition could possibly reconcile the FIR bright-end source count discrepancy between the observations and the model predictions, and will be necessary in deriving the FIR luminosity functions, particularly in the bright end. Our approach provides a much less expensive alternative to doing submillimeter interferometry and has the capability of dealing with a large number of objects upon full automation.
The properly extracted, multi-band FIR fluxes allow us to determine the total IR luminosities with high accuracy, and also enable us to derive other physical parameters in the dusty region, such as the dust temperature, the dust mass and the gas mass. We find that all of these seven objects are ULIRGs $\left(L_{\mathrm{IR}} \geqslant 10^{12} L_{\odot}\right)$ at $z \geqslant 1$. The deep HST images show that they all have very disturbed morphologies, indicating either mergers or violent instability. Using the radio data in these fields, we investigate the FIR-radio relation, and find that it generally holds but still breaks down in a number of cases. However, a proper interpretation of the break-down will have to wait for a much larger sample.

The Herschel FIR bands sample the peak of dust emission over a wide range of temperatures and redshifts, and hence the selection of ULIRG at $z>1$ using the Herschel bands will be more comprehensive and less prone to selection biases. Our limited sample already shows that this is indeed the case. The closest analog to our objects are SMGs, which are known to be biased against high dust temperature. Our small sample includes one ULIRG that has a high dust temperature, which was barely selected by the previous SCUBA survey. The majority of our objects actually are of similar dust temperatures as normal SMG, however, they all fall below the nominal SMG flux selection limit, presumably due to their lower stellar masses that the SMGs.

The detailed SED analysis of the objects in our sample shows that the host galaxies of these ULIRGs at $z \geqslant 1$ are heterogeneous. Within our small sample, the hosts span a wide range in their stellar masses and SFHs. This suggests that an ULIRG phase, if happens, can occur at any stage during the evolution of high-mass galaxies. We provide a new diagnostics of high- $z$ ULIRGs, which utilizes the stellar mass and the age of the host, the ratio of the gas mass to the existing stellar mass, and the ratio of the stellar mass doubling time to the age of the host. This can be used to reveal how efficient and effective the current ULIRG phase is producing stars, and its relation to the existing stellar population. With a much increased sample in the future, it is promising to improve such diagnostics to shed new light on the role of ULIRGs in the global stellar mass assembly.

We thank the anonymous referee for the critical reading and useful comments which helped improve the quality of the 
paper. We thank M. Bolzonella for providing the latest update of the Hyperz code, C. Peng for the useful discussion of the GALFIT code, R. Siebenmorgen for explaining a number of details of the SK07 models, R. Ivison for allowing the use of the non-public radio map in the EGS field, and E. Laird and K. Nandra for the AEGIS Chandra X-ray catalog that is not yet public. We also thank M. Dickinson, H. Ferguson, J. Newman, and M. Salvato for their useful comments. H.Y., M.S., and Z.M. acknowledge the support of Spitzer RSA 1445905. This work is based on observations made by Herschel, an ESA space observatory with science instruments provided by European-led Principal Investigator consortia and with important participation from NASA. Support for Program number HST-GO-12060 was provided by NASA through a grant from the Space Telescope Science Institute, which is operated by the Association of Universities for Research in Astronomy, Incorporated, under NASA contract NAS5-26555. This work is also based in part on observations made with the Spitzer Space Telescope, which is operated by the Jet Propulsion Laboratory, California Institute of Technology under a contract with NASA. Support for this work was provided by NASA through an award issued by JPL/Caltech. This work also makes use of the SDSS. Funding for the creation and distribution of the SDSS Archive has been provided by the Alfred P. Sloan Foundation, the Participating Institutions, the National Aeronautics and Space Administration, the National Science Foundation, the Department of Energy, the Japanese Monbukagakusho, and the Max Planck Society. The SDSS Web site is http://www.sdss.org. The SDSS is managed by the Astrophysical Research Consortium for the Participating Institutions. The Participating Institutions are the University of Chicago, Fermilab, the Institute for Advanced Study, the Japan Participation Group, The Johns Hopkins University, Los Alamos National Laboratory, the Max Planck Institute for Astronomy, the Max Planck Institute for Astrophysics, New Mexico State University, the University of Pittsburgh, Princeton University, the US Naval Observatory, and the University of Washington. This paper has used data obtained by the SHARDS project, funded by the Spanish MINECO grant AYA2012-31277, and based on observations made with the Gran Telescopio Canarias (GTC).

\section{APPENDIX}

\section{PROSPECTIVE AUTOMATION OF THE DECOMPOSITION PROCESS}

The decomposition scheme that we developed in this work has the potential of being automated and being applied to large samples in the future. Specifically, the "automatic iterative" and the "trial-and-error" steps can be integrated, and, in principle, we do not need a mid-IR "ladder" such as an image in MIPS $24 \mu \mathrm{m}$. There are multiple choices of possible approaches that involve different algorithms and criteria, and it will require extensive tests on a large sample before the implement can be finalized. There will be a lot of degenerated cases, and additional information that we can obtain from optical/NIR, such as morphology and $z_{\mathrm{ph}}$, can be used to further narrow the possible solutions. While the automation is beyond the scope of this paper, we show here that it is feasible. We describe one possible approach below for the decomposition in SPIRE $250 \mu \mathrm{m}$

Basically, this approach is a generalization of the "trialand-error" method. It is reasonable to assume that, generally speaking, the objects that are closer to the source centroid are more likely the real contributors. Therefore, we define a core radius from the source centroid, $r_{c}$, and start the decomposition from the objects within this radius. The most optimal choice of this radius will have to wait for extensive tests, and for the illustration purpose here we choose $r_{c}=6^{\prime \prime}$. We first consider the case that there is only one real contributor to all the flux, and the decomposition routine cycles through all the objects within $r_{c}$ one by one. We then consider the case where there are two contributors, and run through all the possible pairs within $r_{c}$. If the secondary contributor in a given solution only accounts for $\leqslant 5 \%$ of the total flux, it is deemed insignificant and the solution falls back to the one-contributor case. The same process repeats for the three-contributor case. While we can add more objects and consider the four-contributor case, in reality this might not be desirable because most of the time the added object within $r_{c}$ will have a separation $\lesssim 2^{\prime \prime}$ to one of the existing object in the group and hence is not going to add an unique solution. For illustration purpose, here we stop at the three-contributor case within $r_{c}$. We then add an object beyond $r_{c}$, cycling through all objects at $r>r_{c}$ one by one, to the one-, two- and three-contributor combinations within $r_{c}$. If a specific addition improves the fit (for example, in terms of $\chi^{2}$ as reported by GALFIT), we add a new object to this combination and repeat the process. Otherwise, we terminate the sequence. Finally, we examine the $\chi^{2}$ values of all the combinations that have been run through. The combinations where an object has its flux error larger than the extracted flux (both as reported by GALFIT) are deemed as "overstretching" and are rejected. We then apply a threshold on $\chi^{2}$ to define a pool of candidate solutions. As our goal is to extract the major component of a given $250 \mu \mathrm{m}$ source, we order these candidate solutions by the major contributor in the solution. One possible way to reach the final answer is to deem the object that has the highest occurrence among all candidate solutions as the major component. In this stage, the additional information from the high-resolution images can be used to help decide on the final choice. It is possible that there will still be degeneracy, and we can offer different interpretations for each should this happen.

Here we use UDS01 as an example to illustrate this approach, where no prior knowledge in $24 \mu \mathrm{m}$ is used. Within $18^{\prime \prime}$ to the $250 \mu \mathrm{m}$ centroid, there are 33 objects in $H_{160}$ with $\mathrm{S} / \mathrm{N} \geqslant 5$ (see Section 5.1.1). Ten of them are within $r_{c}=6^{\prime \prime}$, which are shown in Figure 41 (left). For the sake of simplicity, we concentrate on the cases within $r_{c}$ and do not go beyond in this illustration. The decomposition was run through the one-, two- and three-contributor cases as described above. Figure 41 (right) also shows the histograms of the fitting $\chi^{2}$ (as reported by GALFIT) for these three cases. It is immediately clear that the two- and three-contributor cases produced better solutions than the one-contributor case, which was confirmed by the visual inspection of the residual maps. The inspection also showed that the residual maps with $\chi^{2}>1.75$ were all significantly worse than those that have smaller $\chi^{2}$ values, and therefore we deemed that the best solutions were those with $\chi^{2} \leqslant 1.75$.

The fitting $\chi^{2}$ alone would suggest that the three-component case produced many more better solutions than the twocomponent case does. In particular, there is a high peak at $\chi^{2}=1.72$ in the three-contributor case. However, further examination showed that only two of them were unique solutions and the rest were all "overstretching." Therefore, the best solutions to choose from were the nine two-contributor solutions plus the two surviving three-contributor solutions, all with $\chi^{2} \leqslant 1.75$. Figure 42 shows their residual maps in two panels in order of 

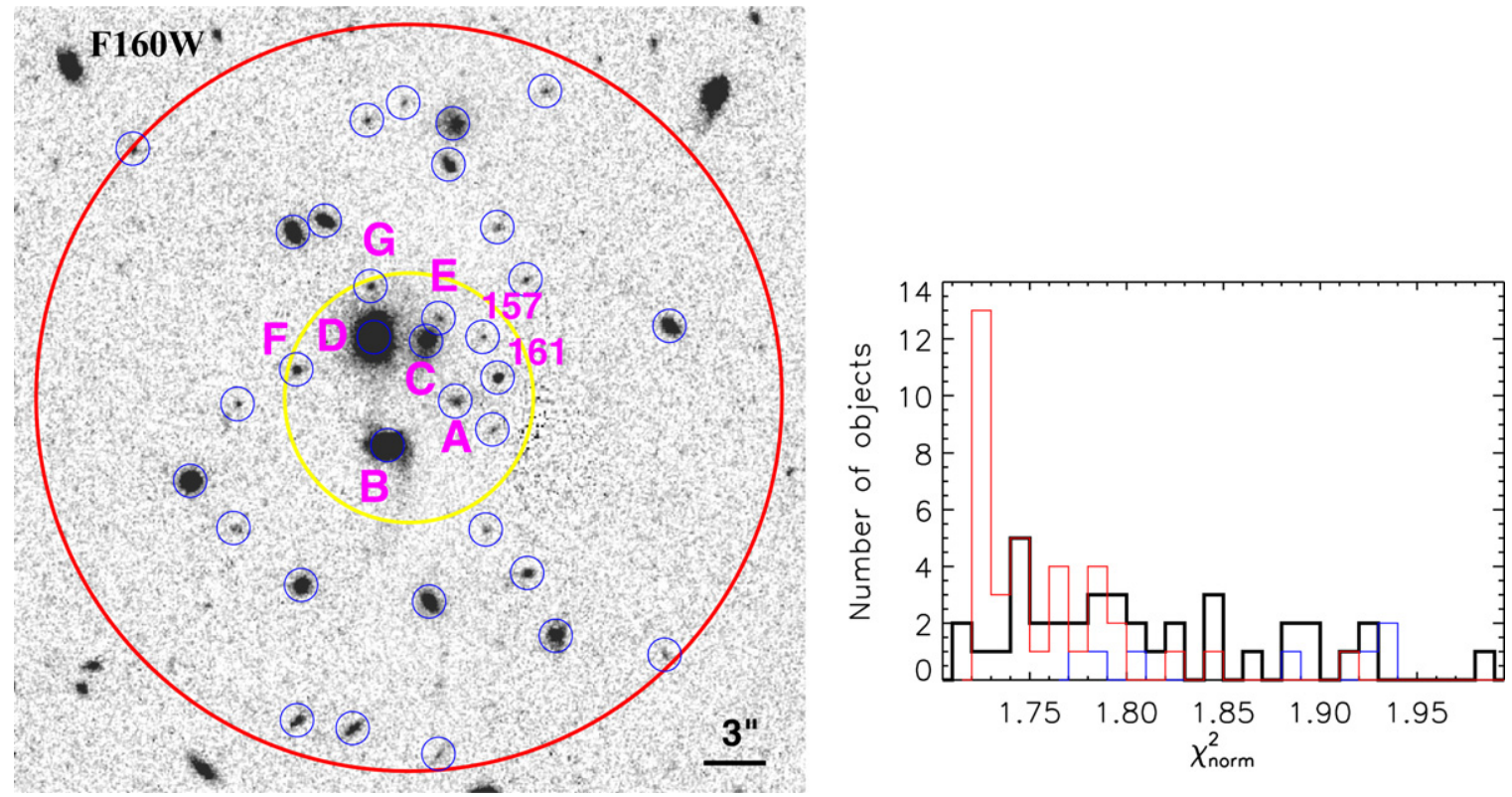

Figure 41. Left: zoomed-in view of UDS01 in $H_{160}$. The red and yellow circles center on the $250 \mu \mathrm{m}$ centroid and are $18^{\prime \prime}$ and $6^{\prime \prime}$ in radius, respectively. The latter indicates the "core region." The objects detected in $H_{160}$ (with $\mathrm{S} / \mathrm{N}>5$ ) are marked by blue circles. The candidate contributors found by the automatic decomposition routine are labeled in magenta, among which those the same as in Section 5.1.1 are labeled the same alphabetically as in Figure 12 and the two additional ones are labeled by numbers. Right: histograms of the fitting $\chi^{2}$ by the automatic routine as described in the text. The one-, two-, and three-contributor cases are coded in blue, black, and red, respectively.

(A color version of this figure is available in the online journal.)
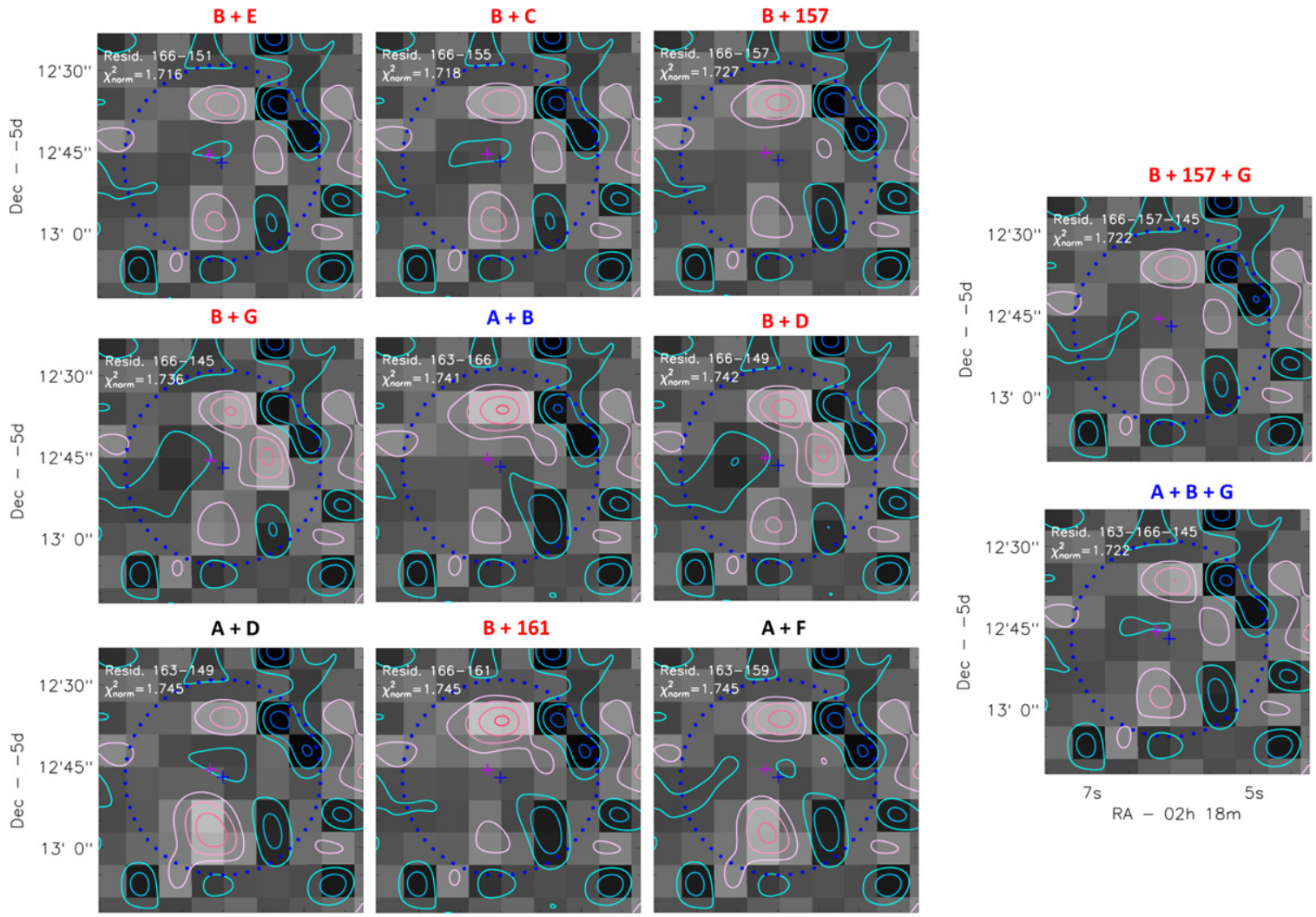

7s 5 s

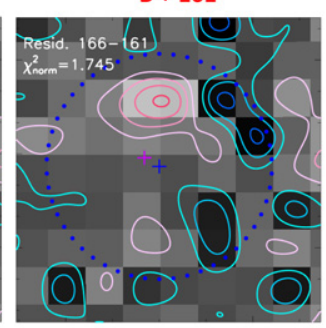

$A+F$
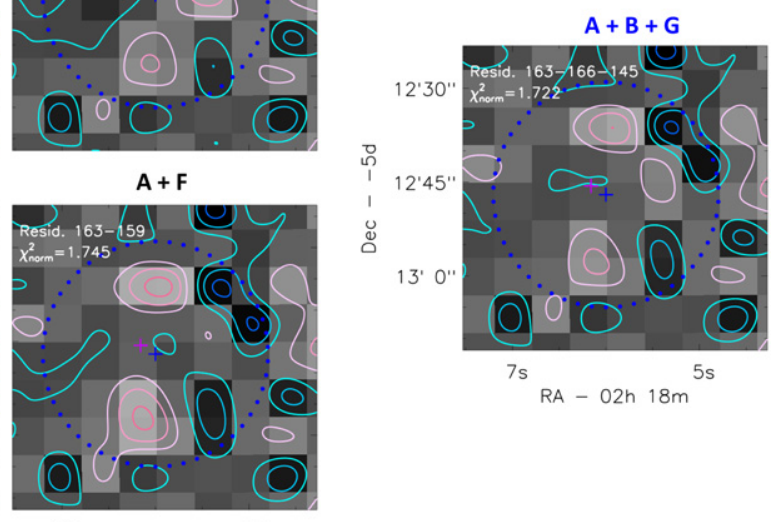

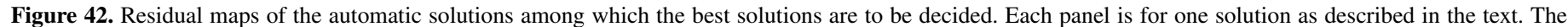

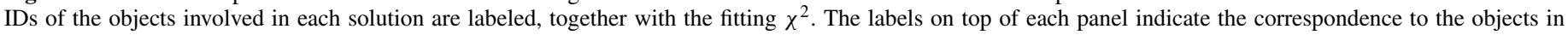
Section 5.1. Other legends are the same as in Figure 15.

(A color version of this figure is available in the online journal.) 
their fitting $\chi^{2}$. The ID (three digit numbers) of the fitted objects are labeled in descending order of their extracted fluxes. To compare to the results in Section 5.1 where the potential contributors were pre-selected using the MIPS $24 \mu \mathrm{m}$ information, we list the correspondence of the relevant ID's here and those in Section 5.1 as follows: $163=$ A, $166=\mathrm{B}, 155=$ $\mathrm{C}, 149=\mathrm{D}, 151=\mathrm{E}, 159=\mathrm{F}$, and $145=\mathrm{G}$. The label on top of each panel in Figure 42 reflects the correspondence. Two conclusions are immediately clear. First, these automated solutions have captured the $24 \mu \mathrm{m}$ pre-selected candidates, and only two objects, 157 and 161, are not among the $24 \mu \mathrm{m}$ pre-selected ones. Second, 9 out of the 11 solutions involve B (166; labeled on top in either red or blue in Figure 42), and 7 of these 9 have it as the major contributor (labeled on top in red in Figure 42). Therefore, our method chose B (166) as the major component of UDS01. The relevant solutions showed that on average B (166) accounted for $61 \pm 6 \%$ of the total flux, agreeing with the value quoted in Table 2 to $2 \%$.

While more difficult, extracting the secondary component is still possible if its contribution is significant. For the case of UDS01, $40 \%$ of the total flux should be accounted for by other objects beyond the major component B (166). To be selfconsistent, the secondary contributor should be sought among the solutions that result in the primary contributor, i.e., the seven solutions shown in Figure 42 with red labels on top. As discussed in Section 5.1.2, C/E (155/151) and D/G (149/145) should be treated as single objects. Therefore, these seven solutions reduce to five, namely, $B+C / E, B+157, B+D / G, B+161$, and $B+157+G$. Note that $B+C / E$ and $B+D / G$ are also the solutions when using $24 \mu \mathrm{m}$ pre-selected candidates as discussed in Section 5.1.3, where we point out that the formal fitting preferred $\mathrm{C} / \mathrm{E}$ but we chose $D / G$ as the final secondary component because of its stronger $24 \mu \mathrm{m}$ flux. Our inspection of UDS01 in $250 \mu \mathrm{m}$ (see Figure 12) indeed showed that the light distribution coincides with $C / E$ better than $D / G$, and in fact this is the reason why objects 157 and 161 were among the solutions because they are closer to side of $C / E$ than to that of $D / G$ (see Figure 41 ). We could not reject 157 or 161 being the secondary based on the decomposition solutions alone, and a proper treatment would need to taking into account other information from optical/NIR images, such as their $z_{\text {ph }}$. This will be deferred to our future paper on the automation. On the other hand, C/E and D/G present an interesting case of degeneracy (given that their $z_{\mathrm{ph}}$ are almost the same), and we could provide different interpretation for each possibility.

\section{REFERENCES}

Alexander, D. M., Bauer, F. E., Schneider, D. P., et al. 2003, AJ, 126, 539 Arnouts, S., Cristiani, S., Moscardini, L., et al. 1999, MNRAS, 310, 540 Ashby, M. L. N., Willner, S. P., Fazio, G. G., et al. 2013, ApJ, 769, 80 Assef, R. J., Kochanek, C. S., Brodwin, M., et al. 2010, ApJ, 713, 970 Barger, A. J., Cowie, L. L., \& Wang, W.-H. 2008, ApJ, 689, 687 Barger, A. J., Wang, W.-H., Cowie, L. L., et al. 2012, ApJ, 761, 89 Barmby, P., Huang, J.-S., Ashby, M. L. N., et al. 2008, ApJS, 177, 431 Bertin, E., \& Arnouts, S. 1996, A\&A, 117, 393

Bolzonella, M., Miralles, J.-M., \& Pelló, R. 2000, A\&A, 363, 476 Borys, C., Smail, I., Blain, A. W., et al. 2005, ApJ, 635, 853

Bradshaw, E. J., Almaini, O., Hartley, W. G., et al. 2013, MNRAS, 433, 194 Bruzual, G., \& Charlot, S. 2003, MNRAS, 344, 1000 (BC03)

Calzetti, D. 2001, PASP, 113, 1449

Capak, P., Carilli, C. L., Lee, N., et al. 2008, ApJL, 681, L53

Casey, C.-M. 2012, MNRAS, 425, 3094

Casey, C.-M., Berta, S., Béthermin, M., et al. 2012, ApJ, 761, 139

Casey, C. M., Chapman, S. C., Beswick, R. J., et al. 2009, MNRAS, 399, 121 Chabrier, G. 2003, PASP, 115, 763

Chapman, S. C., Blain, A. W., Smail, I., \& Ivison, R. J. 2005, ApJ, 622, 772
Chapman, S. C., Ivison, R. J., Roseboom, I. G., et al. 2010, MNRAS, 409, L13 Clements, D. L., Rigby, E., Maddox, S., et al. 2010, A\&A, 518, L8 Condon, J. J. 1992, ARA\&A, 30, 575

Coppin, K. E. K., Smail, I., Alexander, D. M., et al. 2009, MNRAS, 395, 1905 Cowie, L. L., Barger, A. J., Wang, W.-H., \& Williams, J. P. 2009, ApJL, 697, L122

Daddi, E., Dannerbauer, H., Stern, D., et al. 2009, ApJ, 694, 1517

Daddi, E., Dickinson, M., Morrison, G., et al. 2007, ApJ, 670, 156

Davis, M., Guhathakurta, P., Konidaris, N. P., et al. 2007, ApJL, 660, L1

Dekel, A., Sari, R., \& Ceverino, D. 2009, ApJ, 703, 785

Draine, B. T., Dale, D. A., Bendo, G., et al. 2007, ApJ, 663, 866

Dye, S., Eales, S. A., Aretxaga, I., et al. 2008, MNRAS, 386, 1107

Eales, S., Dunne, L., Clements, D., et al. 2010, PASP, 122, 499

Elbaz, D., Daddi, E., Le Borgne, D., et al. 2007, A\&A, 468, 33

Elbaz, D., Dickinson, M., Hwang, H. S., et al. 2011, A\&A, 533, 119

Elbaz, D., Hwang, H. S., Magnelli, B., et al. 2010, A\&A, 518, L29

Elvis, M., Wilkes, B. J., McDowell, J. C., et al. 1994, ApJS, 95, 1

Galametz, M., Madden, S. C., Galliano, F., et al. 2011, A\&A, 532, 56

Georgakakis, A., Rowan-Robinson, M., Babbedge, T. S. R., \& Georgantopoulos, I. 2007, MNRAS, 377, 203

Giavalisco, M., Ferguson, H. C., Koekemoer, A. M., et al. 2003, ApJL, 600, L93

Griffin, M. J., Abergel, A., Abreu, A., et al. 2010, A\&A, 518, L3

Grogin, N. A., Kocevski, D. D., Faber, S. M., et al. 2011, ApJS, 197, 35

Hainline, L. J., Blain, A. W., Smail, I., et al. 2011, ApJ, 740, 96

Hayward, C. C., Behroozi, P., Somerville, R. S., et al. 2013, MNRAS, 434, 2572

Hayward, C. C., Kereš, D., Jonsson, P., et al. 2011, ApJ, 743, 159

Helou, G., Soifer, B. T., \& Rowan-Robinson, M. 1985, ApJL, 298, L7

Ho, P. T. P., Moran, J. M., \& Lo, K. Y. 2004, ApJL, 616, L1

Hodge, J. A., Karim, A., Smail, I., et al. 2013, ApJ, 768, 91

Hughes, D. H., Serjeant, S., Dunlop, J., et al. 1998, Nautr, 394, 241

Ilbert, O., Arnouts, S., McCracken, H. J., et al. 2006, A\&A, 457, 841

Ivison, R. J., Chapman, S. C., Faber, S. M., et al. 2007, ApJL, 660, L77

Ivison, R. J., Magnelli, B., Ibar, E., et al. 2010, A\&A, 518, L31

Karim, A., Schinnerer, E., Martínez-Sansigre, A., et al. 2011, ApJ, 730, 61

Karim, A., Swinbank, A. M., Hodge, J. A., et al. 2013, MNRAS, 432, 2

Kartaltepe, J. S., Dickinson, M., Alexander, D. M., et al. 2012, ApJ, 757, 23

Kennicutt, R. C., Jr. 1998, ApJ, 498, 541

Khan, S. A., Chanial, P. F., Willner, S. P., et al. 2009, ApJ, 706, 319

Koekemoer, A. M., Faber, S. M., Ferguson, H. C., et al. 2011, ApJS, 197, 36

Laidler, V. G., Grogin, N., Clubb, K., et al. 2006, in ASP Conf. Ser. 351,

Astronomical Data Analysis Software and Systems XV, ed. C. Gabriel et al.

(San Francisco, CA: ASP), 228

Laird, E. S., Nandra, K., Georgakakis, A., et al. 2009, ApJS, 180, 102

Leitner, S. N. 2012, ApJ, 745, 149

Leroy, A. K., Bolatto, A., Gordon, K., et al. 2011, ApJ, 737, 12

Lonsdale, C., Farrah, D., \& Smith, H. 2006, in Astrophysics Update 2, ed. J. W. Masona (Chichester: Praxis), 285

Lutz, D., Poglitsch, A., Altieri, B., et al. 2011, A\&A, 532, 90

Madau, P., Pozzetti, L., \& Dickinson, M. 1998, ApJ, 498, 106

Magnelli, B., Lutz, D., Berta, S., et al. 2010, A\&A, 518, L28

Magnelli, B., Lutz, D., Saintonge, A., et al. 2014, A\&A, 561, 86

Magnelli, B., Lutz, D., Santini, P., et al. 2012, A\&A, 539, 155

Magnelli, B., Popesso, P., Berta, S., et al. 2013, A\&A, 554, 132

McLure, R. J., Pearce, H. J., \& Dunlop, J. S. 2013, MNRAS, 428, 1088

Michałowski, M. J., Kamble, A., Hjorth, J., et al. 2012, ApJ, 755, 85

Michałowski, M. J., Watson, D., \& Hjorth, J. 2010, ApJ, 712, 942

Morrison, G. E., Owen, F. N., Dickinson, M., et al. 2010, ApJS, 188, 178

Newman, J. A., Cooper, M. C., Davis, M., et al. 2013, ApJS, 208, 5

Nguyen, H. T., Schulz, B., Levenson, L., et al. 2010, A\&A, 518, L5

Niemi, S.-M., Somerville, R. S., Ferguson, H. C., et al. 2012, MNRAS, 421, 1539

Noeske, K. G., Weiner, B. J., Faber, S. M., et al. 2007, ApJ, 660, L43

Oliver, S. J., Bock, J., Altieri, B., et al. 2012, MNRAS, 424, 1614

Papovich, C., Finkelstein, S. L., Ferguson, H. C., et al. 2011, MNRAS, 412, 1123

Pérez-González, P., Cava, A., Barro, G., et al. 2013, ApJ, 762, 46

Peng, C. Y., Ho, L. C., Impey, C. D., \& Rix, H.-W. 2002, AJ, 124, 266

Pilbratt, G. L., Riedinger, J. R., Passvogel, T., et al. 2010, A\&A, 518, L1

Poglitsch, A., Waelkens, C., Geis, N., et al. 2010, A\&A, 518, L2

Pope, A., Scott, D., Dickinson, M., et al. 2006, MNRAS, 370, 1185

Rawle, T. D., Rex, M., Egami, E., et al. 2012, ApJ, 756, 106

Richards, G. T., Lacy, M., Storrie-Lombardi, L. J., et al. 2006, ApJS, 166, 470

Riechers, D. A., Bradford, C. M., Clements, D. L., et al. 2013, Natur, 496, 329

Rodighiero, G., Daddi, E., Baronchelli, I., et al. 2011, ApJL, 739, L40

Roseboom, I. G., Oliver, S. J., Kunz, M., et al. 2010, MNRAS, 409, 48

Scalo, J. M. 1986, FCPh, 11, 1

Schinnerer, E., Carilli, C. L., Capak, P., et al. 2008, ApJL, 689, L5 
Siebenmorgen, R., \& Krügel, E. 2007, A\&A, 462, 445 (SK07)

Simpson, C., Martínez-Sansigre, A., Rawlings, S., et al. 2006, MNRAS, 372,741

Smith, D. J. B., Dunne, L., Maddox, S. J., et al. 2011, MNRAS, 416, 857

Stetson, P. B. 1987, PASP, 99, 191

Sutherland, W., \& Saunders, W. 1992, MNRAS, 259, 413

Swinbank, A. M., Simpson, J. M., Smail, I., et al. 2014, MNRAS, 438, 1267

Symeonidis, M., Vaccari, M., Berta, S., et al. 2013, MNRAS, 431, 2317

Targett, T. A., Dunlop, J. S., Cirasuolo, M., et al. 2013, MNRAS, 432, 2012

Ueda, Y., Watson, M. G., Stewart, I. M., et al. 2008, ApJS, 179, 124
Walter, F., Decarli, R., Carilli, C., et al. 2012, Natur, 486, 233

Wang, W.-H., Cowie, L. L., \& Barger, A. J. 2004, ApJ, 613, 655

Wang, W.-H., Cowie, L. L., Barger, A. J., \& Williams, J. P. 2011, ApJL, 726, L18

Wang, W.-H., Cowie, L. L., van Saders, J., et al. 2007, ApJL, 670, L89

Willner, S. P., Ashby, M. L. N., Barmby, P., et al. 2012, ApJ, 756, 72

Wuyts, S., Förster Schreiber, N. M., Lutz, D., et al. 2011, ApJ, 738,106

York, D. G., Adelman, J., Anderson, J. E., Jr., et al. 2000, AJ, 120, 1579

Younger, J. D., Fazio, G. G., Huang, J.-S., et al. 2009, ApJ, 704, 803 